\title{
ASHEE-1.0: a compressible, equilibrium-Eulerian model for volcanic ash plumes
}

\author{
M. Cerminara ${ }^{1,2,3}$, T. Esposti Ongaro ${ }^{2}$, and L. C. Berselli ${ }^{3}$ \\ ${ }^{1}$ Scuola Normale Superiore, Pisa, Italy \\ ${ }^{2}$ Istituto Nazionale di Geofisica e Vulcanologia, Sezione di Pisa, Pisa, Italy \\ ${ }^{3}$ Dipartimento di Matematica, Università degli Studi di Pisa, Pisa, Italy \\ Correspondence to: M. Cerminara (matteo.cerminara@gmail.com)
}

Received: 4 September 2015 - Published in Geosci. Model Dev. Discuss.: 19 October 2015

Revised: 20 January 2016 - Accepted: 20 January 2016 - Published: 18 February 2016

\begin{abstract}
A new fluid-dynamic model is developed to numerically simulate the non-equilibrium dynamics of polydisperse gas-particle mixtures forming volcanic plumes. Starting from the three-dimensional N-phase Eulerian transport equations for a mixture of gases and solid dispersed particles, we adopt an asymptotic expansion strategy to derive a compressible version of the first-order non-equilibrium model, valid for low-concentration regimes (particle volume fraction less than $10^{-3}$ ) and particle Stokes number ( $S t-$ i.e., the ratio between relaxation time and flow characteristic time) not exceeding about 0.2. The new model, which is called ASHEE (ASH Equilibrium Eulerian), is significantly faster than the N-phase Eulerian model while retaining the capability to describe gas-particle non-equilibrium effects. Direct Numerical Simulation accurately reproduces the dynamics of isotropic, compressible turbulence in subsonic regimes. For gas-particle mixtures, it describes the main features of density fluctuations and the preferential concentration and clustering of particles by turbulence, thus verifying the model reliability and suitability for the numerical simulation of highReynolds number and high-temperature regimes in the presence of a dispersed phase. On the other hand, Large-Eddy Numerical Simulations of forced plumes are able to reproduce the averaged and instantaneous flow properties. In particular, the self-similar Gaussian radial profile and the development of large-scale coherent structures are reproduced, including the rate of turbulent mixing and entrainment of atmospheric air. Application to the Large-Eddy Simulation of the injection of the eruptive mixture in a stratified atmosphere describes some of the important features of turbulent volcanic plumes, including air entrainment, buoyancy
\end{abstract}

reversal and maximum plume height. For very fine particles ( $S t \rightarrow 0$, when non-equilibrium effects are negligible) the model reduces to the so-called dusty-gas model. However, coarse particles partially decouple from the gas phase within eddies (thus modifying the turbulent structure) and preferentially concentrate at the eddy periphery, eventually being lost from the plume margins due to the concurrent effect of gravity. By these mechanisms, gas-particle non-equilibrium processes are able to influence the large-scale behavior of volcanic plumes.

\section{Introduction}

Explosive volcanic eruptions are characterized by the injection from a vent into the atmosphere of a mixture of gases, liquid droplets and solid particles, at high velocity and temperature. In typical magmatic eruptions, solid particles (also termed tephra) constitute more than $95 \%$ of the erupted mass and are mostly produced by fragmentation of a highly viscous magma during its rapid ascent in a narrow conduit (Wilson, 1976; Sparks, 1978), with particle sizes and densities spanning over a wide range, depending on the overall character and intensity of the eruption (Kaminski and Jaupart, 1998; Kueppers et al., 2006). In this paper, we consider eruptive particles mostly in the sub-millimeter range, generally termed ash. The plume mixture volumetric concentration very rarely exceeds $\epsilon_{\mathrm{s}} \sim 3 \times 10^{-3}$, and the order of magnitude of the ejected fragments density is $\hat{\rho}_{\mathrm{s}} \sim 10^{3} \mathrm{~kg} \mathrm{~m}^{-3}$. Thus, the plume mixture can be considered mainly as a dilute suspension in the sense of Elghobashi (1991, 1994). 
After injection in the atmosphere, this multiphase eruptive mixture can rise convectively in the atmosphere, either forming a buoyant volcanic plume or collapsing catastrophically forming pyroclastic density currents. These two endmembers have different spatial and temporal scales and different impacts on the surrounding of a volcano. Understanding the dynamics of volcanic columns is one of the topical aims of volcanology and the main motivation for this work.

The term volcanic column will be adopted in this paper to generically indicate the eruptive character (e.g., convective/collapsing column). Following the fluid-dynamic nomenclature, we will term jet the inertial regime of the volcanic column and plume the buoyancy-driven regime. A forced plume is characterized by an initial momentumdriven jet stage, transitioning into a plume.

In this work, we present a new computational fluiddynamic model to simulate turbulent gas-particle forced plumes in the atmosphere. Although the focus of the paper is on subsonic regimes, the model is also suited to be applied to transonic and supersonic flows. In many cases, indeed, the eruptive mixture is injected into the atmosphere at pressure higher than atmospheric, so that the flow is initially driven by a rapid, transonic decompression stage. This is suggested by numerical models predicting choked flow conditions at the volcanic vent (Wilson, 1980; Wilson et al., 1980), implying a supersonic transition above the vent or in the crater (Kieffer, 1984; Woods and Bower, 1995; Koyaguchi et al., 2010) and it is supported by field evidences of the emission of shock waves during the initial stages of an eruptions (Morrissey, 1997). Despite the importance of the decompression stage for the subsequent development of the volcanic plume (Pelanti and LeVeque, 2006; Ogden et al., 2008b; Orescanin et al., 2010; Carcano et al., 2013) and for the stability of the eruptive column (Ogden et al., 2008a), our analysis is limited to the plume region where flow pressure is equilibrated to the atmospheric pressure. From laboratory experiments, this is expected to occur within less than 20 inlet diameters above the ground (Yüceil and Ötügen, 2002).

A wide set of numerical tests are presented in this paper (Sect. 4) to assess the adequacy of the model for the intended volcanological application (Sect. 5) and the reliability of the numerical solution method.

\subsection{Dusty-gas modeling of volcanic plumes}

Starting from the assumption that the large-scale behavior of volcanic columns is controlled by the bulk properties of the eruptive mixture, most of the previous models of volcanic plumes have considered the eruptive mixture as homogeneous (i.e., they assume that particles are perfectly coupled to the gas phase). Under such a hypothesis, the multiphase transport equations can be largely simplified and reduce to a set of mass, momentum and energy balance equations for a single fluid (named dusty-gas or pseudo-gas) having average thermo-fluid dynamic properties (mixture density, ve- locity and temperature) and an equation of state accounting for the incompressibility of the particulate phase and gas covolume (Marble, 1970).

By adopting the dusty-gas approximation, volcanic plumes have been studied in the framework of jet (Prandtl, 1963) and plume theory (Morton et al., 1956; Morton, 1959). One-dimensional, steady-state dusty-gas models of volcanic plumes have thus had a formidable role in volcanology to identify the main processes controlling their dynamics and scaling properties (Wilson, 1976; Woods, 1988; Sparks et al., 1997).

Accordingly, volcanic plume dynamics are schematically subdivided into two main stages. The lower, jet phase is driven by the initial flow momentum. Mixture buoyancy is initially negative (the bulk density is larger than atmospheric) but the mixture progressively expands adiabatically thanks to atmospheric air entrainment and heating, eventually undergoing a buoyancy reversal. When buoyancy reversal does not occur, partial or total collapse of the jet from its maximum thrust height and generation of pyroclastic density currents are expected.

Above the jet thrust region, the rise of volcanic plumes is driven by buoyancy and it is controlled by turbulent mixing until, in the stratified atmosphere, a level of neutral buoyancy is reached. Above that level, the plume rises up to its maximum height and then starts to spread out as a gravity current (e.g., Costa et al., 2013) forming an umbrella ash cloud dispersing in the atmosphere and slowly falling-out.

In one-dimensional, time-averaged models, entrainment of atmospheric air is described by one empirical coefficient (the entrainment coefficient) relating the influx of atmospheric air to the local, vertical plume velocity. The entrainment coefficient also determines the plume shape (Ishimine, 2006) and can be empirically assessed by means of direct field observations or ad hoc laboratory measurements.

Further development of volcanic plume models have included the influence of atmospheric stratification and humidity (Woods, 1993; Glaze and Baloga, 1996), the effect of crosswind (Bursik, 2001), loss and re-entrainment of solid particles from plume margins (Woods and Bursik, 1991; Veitch and Woods, 2002), wet aggregation (Folch et al., 2015) and transient effects (Scase, 2009; Woodhouse et al., 2015). However, one-dimensional models strongly rely on the self-similarity hypothesis, whose validity cannot be experimentally ascertained for volcanic eruptions.

To overcome the limitations of one-dimensional models, three-dimensional dusty-gas models have been developed to simulate volcanic plumes. Suzuki (2005) have developed a three-dimensional dusty-gas model (SK-3D) able to accurately resolve the relevant turbulent scales of a volcanic plume, allowing a first, theoretical determination of the entrainment coefficient (Suzuki and Koyaguchi, 2010), without the need of an empirical calibration.

To simulate the three-dimensional large-scale dynamics of volcanic plumes including particle settling and the complex 
microphysics of water in volcanic plumes, the ATHAM (Active Tracer High Resolution Atmospheric Model) code has been designed (Oberhuber et al., 1998; Graf et al., 1999; Van Eaton et al., 2015). ATHAM describes the dynamics of gasparticle mixtures by assuming that particles are in kinetic equilibrium with the gas phase only in the horizontal component, whereas along the vertical direction they are allowed to have a differential velocity. Thermal equilibrium is assumed. In this sense, ATHAM relaxes the dusty-gas approximation (while maintaining its fundamental structure and the same momentum transport equations) by describing the settling of particles with respect to the gas.

\subsection{Multiphase flow models of volcanic plumes}

Notwithstanding all the above advantages, dusty-gas models are still limited by the equilibrium assumption, which can be questionable at least for the coarsest part of the granulometric spectrum in a plume. Turbulence is indeed a nonlinear, multiscale process and the time and space scales of gas-particle interaction may be comparable with some relevant turbulent scales, thus influencing the large-scale behavior of volcanic plumes.

To model non-equilibrium processes, Eulerian multiphase flow models have been developed, which solve the full set of mass, momentum and energy transport equations for a mixture of gas and dispersed particles, treated as interpenetrating fluids. Valentine and Wohletz (1989), Dobran et al. (1993) and Neri and Dobran (1994) first analyzed the influence of erupting parameters on the column behavior. By means of two-dimensional numerical simulations, they individuated a region of transition from collapsing to convective columns. Lately, two-dimensional (Di Muro et al., 2004; Dartevelle et al., 2004) and three-dimensional numerical simulations (Esposti Ongaro et al., 2008) have contributed to further modify the view of a sharp transition between convecting and collapsing columns in favor of that of a transitional regime, characterized by a progressively increasing fraction of mass collapsing. However, previous works could not investigate in detail the non-equilibrium effects in volcanic plumes, mainly because of their averaged description of turbulence: a detailed resolution of the relevant turbulent scales in three dimensions would indeed be computationally prohibitive for $\mathrm{N}$-phase systems.

The main objective of the present work is therefore to develop a new physical model and a fast three-dimensional numerical code able to resolve the spatial and temporal scales of the interaction between gas and particles in turbulent regimes and to describe the kinetic non-equilibrium dynamics and their influence on the observable features of volcanic plumes. To this aim, a development of the so-called equilibriumEulerian approach (Ferry and Balachandar, 2001; Balachandar and Eaton, 2010) has been adopted. It is a generalization of the dusty-gas model keeping the kinematic nonequilibrium as a first-order correction of the Marble (1970) model with respect to the Stokes number of the solid particles in the mixture. Here, we generalize the Ferry and Balachandar (2001) model to the compressible two-way case.

The derivation of the fluid dynamic model describing the non-equilibrium gas-particle mixture is described in detail in Sect. 2. The computational solution procedure and the numerical code development are reported in Sect. 3. Section 4 focuses on verification and evaluation issues in the context of applications to turbulent volcanic plumes. In particular, we discuss three-dimensional numerical simulations of compressible isotropic turbulence (with and without particles), experimental-scale forced plumes and the Sod (1978) shock tube problem. Finally, Sect. 5 presents numerical simulations of volcanic plumes and discusses some aspects related to numerical grid resolution in practical cases.

\section{The multiphase flow model}

To derive an appropriate multiphase flow model to describe gas-particle volcanic plumes, we introduce the nondimensional scaling parameters characterizing gas-particle and particle-particle interactions.

The drag force between gas and particles introduces into the system a timescale $\tau_{\mathrm{s}}$, the particle relaxation time, which is the time a particle needs to equilibrate to a change of gas velocity. Gas-particle drag is a nonlinear function of the local flow variables and, in particular, it depends strongly on the relative Reynolds number, defined as

$R e_{\mathrm{s}}=\frac{\hat{\rho}_{\mathrm{g}}\left|\boldsymbol{u}_{\mathrm{s}}-\boldsymbol{u}_{\mathrm{g}}\right| d_{\mathrm{s}}}{\mu}$.

Here $d_{\mathrm{s}}$ is the particle diameter, $\hat{\rho}_{\mathrm{g}}$ is the gas density, $\mu$ is the gas dynamic viscosity and $\boldsymbol{u}_{\mathrm{g}(\mathrm{s})}$ is the gas (solid) phase velocity field. With $\hat{\rho}_{\mathrm{g}(\mathrm{s})}$ being the gaseous (solid) phase density and $\epsilon_{\mathrm{s}}=V_{\mathrm{s}} / V$ the volumetric concentration of the solid phase, it is useful to define the gas bulk density $\rho_{\mathrm{g}} \equiv\left(1-\epsilon_{\mathrm{s}}\right) \hat{\rho}_{\mathrm{g}} \simeq \hat{\rho}_{\mathrm{g}}$ and the solid bulk density $\rho_{\mathrm{s}} \equiv \epsilon_{\mathrm{s}} \hat{\rho}_{\mathrm{s}}$ (even though in our applications $\epsilon_{\mathrm{s}}$ is order $10^{-3}, \rho_{\mathrm{s}}$ is nonnegligible since $\hat{\rho}_{\mathrm{s}} / \hat{\rho}_{\mathrm{g}}$ is of order $\left.10^{3}\right)$.

For an individual point-like particle (i.e., having diameter $d_{\mathrm{s}}$ much smaller than the scale of the problem under analysis), at $R e_{\mathrm{S}}<1000$, the drag force per volume unity can be given by Stokes' law:

$\boldsymbol{f}_{\mathrm{s}}=\frac{\rho_{\mathrm{s}}}{\tau_{\mathrm{s}}}\left(\boldsymbol{u}_{\mathrm{g}}-\boldsymbol{u}_{\mathrm{s}}\right)$

where

$\tau_{\mathrm{s}} \equiv \frac{\hat{\rho}_{\mathrm{s}}}{\hat{\rho}_{\mathrm{g}}} \frac{d_{\mathrm{s}}^{2}}{18 v \phi_{\mathrm{c}}\left(R e_{\mathrm{s}}\right)}$

is the characteristic time of particle velocity relaxation with respect the gas, $\hat{\rho}_{\mathrm{s}}$ is the particle density, $v$ is the gas kinematic viscosity and $\phi_{\mathrm{c}}=1+0.15 R e_{\mathrm{s}}^{0.687}$ is a correction factor for particle Reynolds number larger than 1 (see Clift 
et al., 1978; Balachandar, 2009; Balachandar and Eaton, 2010; Cerminara, 2016) and for spherical particles (Ganser, 1993). In Eq. (2) we disregard all effects due to the pressure gradient, the added mass, the Basset history and the Saffman terms, because we are considering heavy particles: $\hat{\rho}_{\mathrm{s}} / \hat{\rho}_{\mathrm{g}} \gg 1$ (see Ferry and Balachandar, 2001; Bagheri et al., 2013). Equation (2) has a linear dependence on the fluidparticle relative velocity only when $R e_{\mathrm{s}} \ll 1$, so that $\phi_{\mathrm{c}} \simeq 1$ and the classic Stokes drag expression is recovered. On the other hand, if the relative Reynolds number $R e_{\mathrm{s}}$ grows, nonlinear effects become much more important in Eq. (3). The Clift et al. (1978) empirical relationship used in this work has been used and tested in a number of papers (e.g., Wang and Maxey, 1993; Bonadonna et al., 2002; Balachandar and Eaton, 2010), and it is equivalent to assuming the following gas-particle drag coefficient:

$C_{\mathrm{D}}\left(R e_{\mathrm{S}}\right)=\frac{24}{R e_{\mathrm{S}}}\left(1+0.15 R e_{\mathrm{s}}^{0.687}\right)$.

Wang and Maxey (1993) discussed nonlinear effects due to this correction on the dynamics of point-like particles falling under gravity in a homogeneous and isotropic turbulent surrounding. We recall here the terminal velocity that can be found by setting $\boldsymbol{u}_{\mathrm{g}}=0$ in Eq. (2) is

$\boldsymbol{w}_{\mathrm{s}}=\sqrt{\frac{4 d_{\mathrm{s}} \hat{\rho}_{\mathrm{s}}}{3 C_{\mathrm{D}} \rho_{\mathrm{g}} g}} \boldsymbol{g}=\tau_{\mathrm{s}} \boldsymbol{g}$.

As previously pointed out, the correction used in Eq. (4) is valid if $R e_{\mathrm{S}}<10^{3}$, the regime addressed in this work for ash particles much denser then the surrounding fluid and smaller than about $1 \mathrm{~mm}$. As shown by Balachandar (2009), maximum values of $R e_{\mathrm{S}}$ are associated with particle gravitational settling (not with turbulence). Using Eqs. (4) and (5), it is thus possible to estimate the $R e_{\mathrm{S}}$ of a falling particle with diameter $d_{\mathrm{s}}$. We obtain that $R e_{\mathrm{S}}$ is always smaller than $10^{3}$ for ash particles finer than $1 \mathrm{~mm}$ in air. If regimes with a stronger decoupling need to be explored, more complex empirical corrections have to be used for $\phi_{\mathrm{c}}$ (Neri et al., 2003; Bürger and Wendland, 2001). It is also worth noting that ash particles can differ significantly from spheres and terminal settling velocities of volcanic particles can be up to a factor 2-3 with respect to spherical assumption. To account for this effect various modifications to Eq. (4) have been devised (e.g., Dellino et al., 2005; Pfeiffer et al., 2005).

The same reasoning can be applied to estimate the thermal relaxation time between gas and particles. In terms of the solid phase specific heat capacity $C_{\mathrm{s}}$ and its thermal conductivity $k_{\mathrm{g}}$, we have

$\tau_{T}=\frac{2}{N u_{\mathrm{s}}} \frac{\hat{\rho}_{\mathrm{s}} C_{\mathrm{s}}}{k_{\mathrm{g}}} \frac{d_{\mathrm{s}}^{2}}{12}$,

where $N u_{\mathrm{s}}=N u_{\mathrm{s}}\left(R e_{\mathrm{s}}, \operatorname{Pr}\right)$ is the Nusselt number, usually function of the relative Reynolds number and of the Prandtl number of the carrier fluid (Neri et al., 2003). In terms of $\tau_{T}$, the heat exchange between a particle at temperature $T_{\mathrm{S}}$ and the surrounding gas at temperature $T_{\mathrm{g}}$ per unit volume is

$\mathrm{Q}_{\mathrm{s}}=\frac{\rho_{\mathrm{s}} C_{\mathrm{s}}}{\tau_{T}}\left(T_{\mathrm{s}}-T_{\mathrm{g}}\right)$.

Comparing the kinetic and thermal relaxation times we get

$\frac{\tau_{T}}{\tau_{\mathrm{s}}}=\frac{3}{2} \frac{2 \phi_{\mathrm{c}}}{N u_{\mathrm{s}}} \frac{C_{\mathrm{s}} \mu}{k_{\mathrm{g}}}$.

In order to estimate this number, firstly we notice that factor $2 \phi_{\mathrm{c}} / N u_{\mathrm{S}}$ tends to 1 if $R e_{\mathrm{s}} \rightarrow 0$, and it remains smaller than $\simeq 2$ if $R e_{\mathrm{s}}<10^{3}$ (Neri et al., 2003; Cerminara, 2016). Then, in the case of ash particles in air, we have (in SI units) $\mu \simeq$ $10^{-5}, C_{\mathrm{s}} \simeq 10^{3}, k_{\mathrm{g}} \simeq 10^{-2}$. Thus we have that $\tau_{T} / \tau_{\mathrm{s}} \simeq 1$, meaning that the thermal equilibrium time is typically of the same order of magnitude as the kinematic one. This bound is very useful when we write the equilibrium-Eulerian and the dusty-gas models, because it ensures that the thermal Stokes number is of the same order as the kinematic one, at least for volcanic ash finer than about $1 \mathrm{~mm}$.

The non-dimensional Stokes number $(S t)$ is defined as the ratio between the kinetic relaxation time and a characteristic time of the flow under investigation $\tau_{L}$, namely $S t_{\mathrm{S}}=\tau_{\mathrm{S}} / \tau_{L}$. The definition of the flow timescale can be problematic for high-Reynolds number flows (typical of volcanic plumes), which are characterized by a wide range of interacting length- and timescales, a distinctive feature of the turbulent regime. For volcanic plumes, the more energetic timescale would be of the order of $\tau_{L}=L / U$, where $L$ and $U$ are the plume diameter and velocity at the vent, which gives the characteristic turnover time of the largest eddies in a turbulent plume (e.g., Zhou et al., 2001). On the other hand, the smallest timescale (largest $S t_{\mathrm{s}}$ ) can be defined by the Kolmogorov similarity law by $\tau_{\eta} \sim \tau_{L} R e_{L}^{-1 / 2}$, where the macroscopic Reynolds number is defined, at first instance, by $R_{L}=U L / v$, with $v$ being the kinematic viscosity of the gas phase numerical models. It is also useful to introduce the Large-Eddy Simulation (LES) timescale $\tau_{\xi}$, relative to the LES length scale $\xi$. This is related to the numerical grid resolution, size of the explicit filter and discretization accuracy (Lesieur et al., 2005; Garnier et al., 2009; Balachandar and Eaton, 2010; Cerminara et al., 2015). At LES scale $\xi, S t_{\mathrm{S}}$ is not as large as at the Kolmogorov scale, thus the decoupling between particles and the carrier fluid is mitigated by the LES filtering operation. Dimensional analysis shows (see Sect. 5) that $S t_{\mathrm{s}} \lesssim 0.2$ for LES of volcanic ash finer than about $1 \mathrm{~mm}$.

The model presented here is conceived for resolving $d i$ lute suspensions, namely mixtures of gases and particles with volumetric concentration $\frac{V_{\mathrm{s}}}{V} \equiv \epsilon_{\mathrm{s}} \lesssim 10^{-3}$. We here use the definition of dilute suspension by Elghobashi $(1991,1994)$ and Balachandar (2009), corresponding to regimes in which particle-particle collisions can be disregarded. This can also be justified by analyzing the timescale of particle-particle 
collisions. In the dilute regime, in which we can assume an equilibrium Maxwell distribution of particle velocities, the mean free path of solid particles is given by (Gidaspow, 1994)

$\lambda_{\mathrm{p}-\mathrm{p}}=\frac{1}{6 \sqrt{2}} \frac{d_{\mathrm{s}}}{\epsilon_{\mathrm{s}}}$.

Consequently, particle-particle collisions are relatively infrequent $\left(\lambda_{\mathrm{p}-\mathrm{p}} \sim 0.1 \mathrm{~m} \gg d_{\mathrm{s}}\right)$, so that we can neglect, as a first approximation, particle-particle collisions and consider the particulate fluid as pressure-less, inviscid and nonconductive.

In volcanic plumes the particle volumetric concentration can exceed by one order of magnitude the threshold $\epsilon_{\mathrm{s}} \simeq 10^{-3}$ only near the vent (see, e.g., Sparks et al., 1997; Esposti Ongaro et al., 2008). However, the region of the plume where the dilute suspension requirement is not fulfilled remains small with respect the size of the entire plume, weakly influencing its global dynamics. Indeed, as we will show in Sect. 5, air entrainment and particle fallout induce a rapid decrease of the volumetric concentration. In contrast, the mass fraction of the solid particles cannot be considered small, because particles are heavy: $\epsilon_{\mathrm{s}} \hat{\rho}_{\mathrm{s}} \equiv \rho_{\mathrm{s}} \simeq \rho_{\mathrm{g}}$. Thus, particle inertia will be considered in the present model: in other words, we will consider the two-way coupling between dispersed particles and the carrier gas phase.

Summarizing, our multiphase model focuses and carefully takes advantage of the hypotheses characterizing the following regimes: heavy particles $\left(\hat{\rho}_{\mathrm{s}} / \hat{\rho}_{\mathrm{g}} \gg 1\right)$ in dilute suspension $\left(\epsilon_{\mathrm{s}} \lesssim 10^{-3}\right)$ with dynamical length scales much larger than the particles diameter (point-particle approach) and relative Reynolds number smaller than $10^{3}$.

\subsection{The Eulerian model in "mixture" formulation}

When $S t \leq 1$ and the number of particles is very large, it is convenient to use an Eulerian approach, where the carrier and the dispersed phases are modeled as interpenetrating continua, and their dynamics is described by the laws of fluid mechanics (Balachandar and Eaton, 2010).

Here we model a polydisperse mixture of $i \in[1,2, \ldots, I] \equiv \mathcal{I}$ gaseous phases and $j \in[I+1, I+$ $2, \ldots, I+J] \equiv \mathcal{J}$ solid phases. From now on, we will use the subscript $(\cdot)_{j}$ instead of $(\cdot)_{\mathrm{S}}$ for the $j$ th solid phase. Thus, the bulk density of the mixture reads: $\rho_{\mathrm{m}}=\sum_{\mathcal{I}} \rho_{i}+\sum_{\mathcal{J}} \rho_{j}$. The mass fractions will be denoted by the symbol $y-$ i.e., $y_{j}=\rho_{j} / \rho_{\mathrm{m}}$. The bulk density of the gas phase thus is $\rho_{\mathrm{g}}=\sum_{\mathcal{I}} \rho_{i}=\sum_{\mathcal{I}} y_{i} \rho_{\mathrm{m}}$, while that of the solid phases $\rho_{\mathrm{s}}=\sum_{\mathcal{J}} \rho_{j}=\sum_{\mathcal{J}} y_{j} \rho_{\mathrm{m}}$. Thus, $\rho_{\mathrm{m}}=\rho_{\mathrm{g}}+\rho_{\mathrm{s}}$. The volumetric concentration of the $i$ th $\left(j\right.$ th) phase is given by $\epsilon_{i}=\rho_{i} / \hat{\rho}_{i}$. Solid phases represent the discretization of a virtually continuous grain-size distribution into discrete bins, as usually done in volcanological studies (see Cioni et al., 2003; Neri et al., 2003). Another possible approach is the method of moments, in which the evolution of the moments of the grain size distribution is described. This has recently been applied in volcanology to integral plume models by de' Michieli Vitturi et al. (2015). In the present work we opted for the classical discretization of the grain size distribution (see Neri et al., 2003). In Cerminara (2016), we analyze the Eulerian-Eulerian model under the barotropic regime to show the existence of weak solutions of the corresponding partial differential equations problem.

In the regime described above, the Eulerian-Eulerian equations for each phase (either gaseous or solid) are written. In Appendix B we reformulate the Eulerian-Eulerian model in a convenient equivalent formulation ("mixture" formulation). We use the subscripts $i, j, \mathrm{~m}$ to associate a generic field $\psi$ with the gas phases $\left(\psi_{i}\right)$, with the solid phases $\left(\psi_{j}\right)$, and with the mixture $\left(\psi_{\mathrm{m}}=\sum_{\mathcal{I}} y_{i} \psi_{i}+\sum_{\mathcal{J}} y_{j} \psi_{j}\right)$. All the fields are defined in a spatial domain $x \in \Omega$ and a temporal interval $t \in \mathcal{T}$. The field variables to be solved are: the density of the mixture $\rho_{\mathrm{m}}(\boldsymbol{x}, t)$; the mass fractions $y_{i}(\boldsymbol{x}, t)$ and $y_{j}(\boldsymbol{x}, t)$; the velocity fields $\boldsymbol{u}_{\mathrm{m}}(\boldsymbol{x}, t)$ and $\boldsymbol{u}_{j}(\boldsymbol{x}, t)$; the enthalpy $h_{\mathrm{m}}(\boldsymbol{x}, t)$; the temperature fields $T_{j}(\boldsymbol{x}, t)$.

The Eulerian-Eulerian model in mixture formulation thus reads

$$
\begin{aligned}
& \partial_{t} \rho_{\mathrm{m}}+\nabla \cdot\left(\rho_{\mathrm{m}} \boldsymbol{u}_{\mathrm{m}}\right)=\sum_{j \in \mathcal{J}} S_{j} ; \\
& \partial_{t}\left(\rho_{\mathrm{m}} y_{i}\right)+\nabla \cdot\left(\rho_{\mathrm{m}} \boldsymbol{u}_{\mathrm{g}} y_{i}\right)=0, \quad i \in \mathcal{I} ; \\
& \partial_{t}\left(\rho_{\mathrm{m}} y_{j}\right)+\nabla \cdot\left(\rho_{\mathrm{m}} \boldsymbol{u}_{j} y_{j}\right)=S_{j}, \quad j \in \mathcal{J} ; \\
& \partial_{t}\left(\rho_{\mathrm{m}} \boldsymbol{u}_{\mathrm{m}}\right)+\nabla \cdot\left(\rho_{\mathrm{m}} \boldsymbol{u}_{\mathrm{m}} \otimes \boldsymbol{u}_{\mathrm{m}}+\rho_{\mathrm{m}} \mathbb{T}_{\mathrm{r}}\right) \\
& =-\nabla p+\nabla \cdot \mathbb{T}+\rho_{\mathrm{m}} \boldsymbol{g}+\sum_{j \in \mathcal{J}} S_{j} \boldsymbol{u}_{j} ; \\
& \partial_{t}\left(\rho_{\mathrm{m}} h_{\mathrm{m}}\right)+\nabla \cdot\left[\rho_{\mathrm{m}} h_{\mathrm{m}}\left(\boldsymbol{u}_{\mathrm{m}}+\boldsymbol{v}_{h}\right)\right]=\partial_{t} p-\partial_{t}\left(\rho_{\mathrm{m}} K_{\mathrm{m}}\right) \\
& -\nabla \cdot\left[\rho_{\mathrm{m}} K_{\mathrm{m}}\left(\boldsymbol{u}_{\mathrm{m}}+\boldsymbol{v}_{K}\right)\right]+\nabla \cdot\left(\mathbb{T} \cdot \boldsymbol{u}_{\mathrm{g}}-\boldsymbol{q}\right)+\rho_{\mathrm{m}}\left(\boldsymbol{g} \cdot \boldsymbol{u}_{\mathrm{m}}\right) \\
& +\sum_{j \in \mathcal{J}} S_{j}\left(h_{j}+K_{j}\right) .
\end{aligned}
$$

The terms $K_{\mathrm{g}}=\frac{1}{2}\left|\boldsymbol{u}_{\mathrm{g}}\right|^{2}$ and $K_{j}=\frac{1}{2}\left|\boldsymbol{u}_{j}\right|^{2}$ are the kinetic energy per unity of mass of the gaseous and solid phases, respectively. The acceleration due to gravity is $g$. The other terms, needing closure constitutive equations, are: the pressure of the gas phase $p$; the stress tensor of the gas phase $\mathbb{T}$; the heat flux in the gas phase $\boldsymbol{q}$; the source (or sink) term for the $j$ th phase $S_{j}$.

The first equation is redundant, because it is contained in the second and third set of continuity equations and $\sum_{\mathcal{I}} y_{i}+\sum_{\mathcal{J}} y_{j}=1$. The system is missing the $4 J$ momentum and enthalpy equations for the solid phases, needed to find the decoupling velocity $\boldsymbol{v}_{j}=\boldsymbol{u}_{j}-\boldsymbol{u}_{\mathrm{g}}$ and the decoupling temperature $T_{j}-T_{\mathrm{g}}$. They are reported in Eq. (B1). 
The equilibrium-Eulerian model described in the Sect. 2.2 provides a way to solve the decoupling equations in a fast, explicit way, avoiding the need to solve the complete system of PDEs of the Eulerian-Eulerian model.

\subsubsection{Constitutive equations}

To close the system, constitutive equations are needed. They are listed here:

- Perfect gas: $p=\sum_{\mathcal{I}} \hat{\rho}_{i} R_{i} T_{\mathrm{g}}$, with $R_{i}$ the gas constant of the $i$ th gas phase. This law can be simplified by nothing that $\epsilon_{\mathrm{S}} \ll 1$, thus $\epsilon_{i} \simeq 1$ and $\hat{\rho}_{i} \simeq \rho_{i}$ (see Suzuki, 2005). Anyway, in this work we use the complete version of the perfect gas law. It can be written in convenient form for a poly-disperse mixture as

$$
\frac{1}{\rho_{\mathrm{m}}}=\sum_{j \in \mathcal{J}} \frac{y_{j}}{\hat{\rho}_{j}}+\sum_{i \in \mathcal{I}} \frac{y_{i} R_{i} T_{\mathrm{g}}}{p} .
$$

- Newtonian gas stress tensor:

$$
\mathbb{T}=2 \mu\left(T_{\mathrm{g}}\right)\left(\operatorname{sym}\left(\nabla \boldsymbol{u}_{\mathrm{g}}\right)-\frac{1}{3} \nabla \cdot \boldsymbol{u}_{\mathrm{g}} \mathbb{I}\right),
$$

where $\mu(T)=\sum_{\mathcal{I}^{\mathcal{X}_{i}} \mu_{i}}(T)$ is the gas dynamic viscosity, $\mu_{i}$ is that of the $i$ th gas component, and $\varkappa_{i}$ is the molar fraction of the $i$ th component (see Graham, 1846).

- Enthalpy per unit of mass of the gas (solid) phase: $h_{\mathrm{g}}=\sum_{\mathcal{I}} \rho_{i} C_{i} T_{\mathrm{g}} / \rho_{\mathrm{g}}+p / \rho_{\mathrm{g}}\left(h_{j}=C_{j} T_{j}\right)$, with $C_{i}\left(C_{j}\right)$ the specific heat at constant volume of the $i$ th ( $j$ th) phase. Thus: $h_{i}=C_{i} T_{\mathrm{g}}+p / \rho_{g} ; \sum_{\mathcal{I}} y_{i} h_{i}=y_{\mathrm{g}} h_{\mathrm{g}}$; $h_{\mathrm{m}}=\sum_{\mathcal{I}} y_{i} C_{i} T_{\mathrm{g}}+\sum_{\mathcal{J}} y_{j} C_{j} T_{j}+p / \rho_{\mathrm{m}}$.

- The Fourier law for the heat transfer in the gas: $\boldsymbol{q}=-k_{\mathrm{g}} \nabla T$, where $k_{\mathrm{g}}=\sum_{\mathcal{I} \varkappa_{i}} k_{i}$ and $k_{i}$ is the conductivity of the $i$ th gas component.

- $S_{j}$ is the source or sink term (when needed) of the $j$ th phase. $K_{i}=\left|\boldsymbol{u}_{i}\right|^{2} / 2$ is the kinetic energy per unit of mass of the $i$ th gas phase ( $K_{j}$ for the $j$ th solid phase).

\subsection{Equilibrium-Eulerian model}

In the limit $S t_{j} \ll 1$, the drag terms $\boldsymbol{f}_{j}$ and the thermal exchange terms $\mathrm{Q}_{j}$ can be calculated by knowing $\boldsymbol{u}_{\mathrm{g}}$ and $T_{\mathrm{g}}$ only, and the Eulerian-Eulerian model can be largely simplified by considering the dusty-gas approximation (Marble, 1970). In this approximation, the coupling is so strong that the decoupling terms $\boldsymbol{v}_{j}$ and $T_{j}-T_{\mathrm{g}}$ go to zero, making it unnecessary to solve the $4 J$ equations for the decoupling in Eq. (10).

A refinement of the dusty-gas approximation (valid if $S t_{j} \lesssim 0.2$ ), has been developed by Maxey (1987) and is discussed in what follows.

\subsubsection{Kinematic decoupling}

The Lagrangian particle momentum balance (see Eq. B1d for its Eulerian counterpart) reads

$\partial_{t} \boldsymbol{u}_{j}+\boldsymbol{u}_{j} \cdot \nabla \boldsymbol{u}_{j}=\frac{1}{\tau_{j}}\left(\boldsymbol{u}_{\mathrm{g}}-\boldsymbol{u}_{j}\right)+\boldsymbol{g}$.

By using the Stokes law and a perturbation method (see Appendix C), and by defining $\boldsymbol{a} \equiv \mathrm{D}_{t} \boldsymbol{u}_{\mathrm{g}}$ (with $\mathrm{D}_{t}=\partial_{t}+\boldsymbol{u} \cdot \nabla$ ), we obtain a correction to particle velocity up to first order:

$\boldsymbol{u}_{j}=\boldsymbol{u}_{\mathrm{g}}+\boldsymbol{w}_{j}-\tau_{j}\left(\partial_{t} \boldsymbol{u}_{\mathrm{g}}+\boldsymbol{u}_{j} \cdot \nabla \boldsymbol{u}_{\mathrm{g}}\right)+O\left(\tau_{j}^{2}\right)$.

It can be restated that

$\boldsymbol{u}_{j}=\boldsymbol{u}_{\mathrm{g}}+\mathbb{G}_{j}^{-1} \cdot\left(\boldsymbol{w}_{j}-\tau_{j} \boldsymbol{a}\right)+O\left(\tau_{j}^{2}\right)$

$\mathbb{G}_{j} \equiv \mathbb{I}+\tau_{j} \alpha\left(\nabla \boldsymbol{u}_{\mathrm{g}}\right)^{\mathrm{T}}$,

leading to the so-called equilibrium-Eulerian model developed by Ferry and Balachandar (2001), Ferry et al. (2003) and Balachandar and Eaton (2010) for incompressible multiphase flows. Here $\alpha$ is a local correction coefficient inserted to avoid singularities (introduced by Ferry et al., 2003). At the zeroth order we recover $\boldsymbol{u}_{j}=\boldsymbol{u}_{\mathrm{g}}+\boldsymbol{w}_{j}$, where $\boldsymbol{w}_{j}$ is the settling velocity defined in Eq. (5).

It is worth noting from Eq. (3) that $\tau_{j}$ depends on $R e_{j}$, and it cannot be determined until $\boldsymbol{u}_{j}$ is known. One solution to this problem has been proposed by Ferry and Balachandar (2002), where $\tau_{j}$ is evaluated directly by knowing

$\widetilde{R} e_{j} \equiv \frac{\hat{\rho}_{j} d_{j}^{3}|\boldsymbol{a}-\boldsymbol{g}|}{18 v}$,

by approximating Eq. (3) in the range $0<R e_{j}<300$. We here improve that approximation in the range $0<R e_{j}<10^{3}$ by using the inversion formula:

$R e_{j}=\frac{\widetilde{\operatorname{Re}} e_{j}}{1+0.315 \widetilde{\operatorname{Re}_{j}^{0.4072}}}$.

Another strategy we use in ASHEE, is to evaluate $R e_{j}$ explicitly from the previous time step and correct it iteratively (within the PISO loop, see Sect. 3.2).

Equation (15) highly simplifies the Eulerian-Eulerian Eq. (B1) because it gives an explicit approximation of the decoupling velocity $\boldsymbol{v}_{j}$, which can be used directly in Eq. (10). There, we keep the term $\nabla \cdot\left(\rho_{\mathrm{m}} \mathbb{T}_{\mathrm{r}}\right)$ because of the presence of the settling velocity $\boldsymbol{w}_{j}$ in $\boldsymbol{v}_{j}$ which is at the leading order.

\subsubsection{Thermal decoupling}

As pointed out in Eq. (8) and below, in our physical regime the thermal Stokes time is of the same order of magnitude as the kinematic one. However, this regime has been thoroughly analyzed in the incompressible case by Ferry and 
Balachandar (2005), demonstrating that the error made by assuming thermal equilibrium is at least one order of magnitude smaller than that on the momentum equation (at equal Stokes number), thus justifying the limit $T_{j} \rightarrow T_{\mathrm{g}}=T$ as done for the thermal equation in the dusty-gas model.

This approximation allows to write in a convenient way the constitutive equation for the enthalpy:

$$
\begin{aligned}
h_{\mathrm{m}} & =C_{\mathrm{m}} T+\frac{p}{\rho_{\mathrm{m}}} \\
C_{\mathrm{m}} & =\sum_{i \in \mathcal{I}} y_{i} C_{i}+\sum_{j \in \mathcal{J}} y_{j} C_{j} .
\end{aligned}
$$

\subsubsection{Advantages of the equilibrium-Eulerian model}

Summarizing, in ASHEE we refer to the compressible equilibrium-Eulerian model as the PDEs listed in Eq. (10) with the constitutive equations described in Sect. 2.1.1, the decoupling velocity $\boldsymbol{v}_{j}=\boldsymbol{u}_{j}-\boldsymbol{u}_{\mathrm{g}}$ written in Eq. (15), and nil thermal decoupling $T_{j}-T_{\mathrm{g}}=0$.

It is worth noting that in the Navier-Stokes equations it is critical to accurately take into account the nonlinear term $\nabla \cdot(\rho \boldsymbol{u} \otimes \boldsymbol{u})$ because it is the origin of the major difficulties in turbulence modeling. A large advantage of the dusty-gas and equilibrium-Eulerian models is that in both models the most relevant part of the drag $\left(\sum_{\mathcal{J}} \boldsymbol{f}_{j}\right)$ and heat exchange $\left(\sum_{\mathcal{J}} \mathrm{Q}_{j}\right)$ terms have been absorbed into the conservative derivatives for the mixture. This fact allows the numerical solver to implicitly and accurately solve the particles' contribution to mixture momentum and energy (two-way coupling), using the same numerical techniques developed in Computational Fluid Dynamics for the Navier-Stokes equations. The dusty-gas and equilibrium-Eulerian models are best suited for solving multiphase system in which the particles are strongly coupled with the carrier fluid and the bulk density of the particles is not negligible with respect to that of the fluid.

The equilibrium-Eulerian model thus reduces to a set of mass, momentum and energy balance equations for the gasparticle mixture plus the equations for the mass transport of the particulate and gaseous phases. In this respect, it is similar to the dusty-gas equations, to which it reduces for $\tau_{\mathrm{s}} \equiv 0$. With respect to the dusty-gas model, the ASHEE model solves for the mixture velocity $\boldsymbol{u}_{\mathrm{m}}$, which is slightly different from the carrier gas velocity $\boldsymbol{u}_{\mathrm{g}}$. Moreover, it can compute the kinematic decoupling (i.e., the difference between the fields $\boldsymbol{u}_{\mathrm{g}}$ and $\boldsymbol{u}_{j}$ ), responsible for preferential concentration and settling phenomena (the vector $\boldsymbol{v}_{j}$ includes a convective and a gravity accelerations terms).

The equilibrium-Eulerian method becomes even more efficient (relative to the standard Eulerian-Eulerian) for the polydisperse case $(J>1)$. For each bin of particle tracked, the standard Eulerian method requires four scalar fields; the fast method requires one. Furthermore, the computation of the correction to $\boldsymbol{v}_{j}$ needs only to be done for one particle species. The correction has the form $-\tau_{j} \boldsymbol{a}$, so once the term $\boldsymbol{a}$ is computed, velocities for all species of particles may be obtained simply by scaling the correction factor based on the species' response times $\tau_{j}$. As already stated, the standard Eulerian method needs $I+4+5 J$ scalar partial differential equations, while the equilibrium-Eulerian model needs just $I+4+J-$ i.e., $4 J$ equations less.

\subsection{LES formulation}

The spectrum of the density, velocity and temperature fluctuations of turbulent flows at high Reynolds number typically spans over many orders of magnitude. When the smallest turbulent length scales cannot be resolved by the numerical grid, it is necessary to model the effects of the high-frequency fluctuations on the resolved flow. This leads to the LES technique, in which a low-pass filter is applied to the model equations to filter out the small scales of the solution. In the incompressible case the theory is well developed (see Berselli et al., 2005; Sagaut, 2006), but LES for compressible flows is still an open research field. In ASHEE, we apply a spatial filter, denoted by an overbar (here $\delta$ is the filter scale):

$\bar{\psi}=\int_{\Omega} G\left(\boldsymbol{x}-\boldsymbol{x}^{\prime} ; \delta\right) \psi\left(\boldsymbol{x}^{\prime}\right) \mathrm{d} \boldsymbol{x}^{\prime}$.

Some example of LES filters $G(x ; \delta)$ used in compressible turbulence are reviewed in Garnier et al. (2009). In compressible turbulence it is also useful to introduce the so-called Favre filter:

$\widetilde{\psi}=\frac{\overline{\rho_{\mathrm{m}} \psi}}{\bar{\rho}_{\mathrm{m}}}$

In Appendix D, we apply this filter to Eq. (15), in order to obtain the LES filtered version of the equilibrium-Eulerian model, Eq. (D3).

By applying the Favre filter to Eq. (10) (for the application of the Favre filter to the compressible Navier-Stokes equations see Garnier et al., 2009, Moin et al., 1991 and Erlebacher et al., 1990), we obtain

$$
\begin{aligned}
& \partial_{t} \bar{\rho}_{\mathrm{m}}+\nabla \cdot\left(\bar{\rho}_{\mathrm{m}} \widetilde{\boldsymbol{u}}_{\mathrm{m}}\right)=\sum_{j \in \mathcal{J}} \widetilde{S}_{j} ; \\
& \partial_{t}\left(\bar{\rho}_{\mathrm{m}} \widetilde{y}_{i}\right)+\nabla \cdot\left(\bar{\rho}_{\mathrm{m}} \widetilde{\boldsymbol{u}}_{\mathrm{g}} \widetilde{y}_{i}\right)=-\nabla \cdot \mathcal{Y}_{i}, \quad i \in \mathcal{I} ; \\
& \partial_{t}\left(\bar{\rho}_{\mathrm{m}} \widetilde{y}_{j}\right)+\nabla \cdot\left[\bar{\rho}_{\mathrm{m}} \widetilde{\boldsymbol{u}}_{j} \widetilde{y}_{j}\right]=\widetilde{S}_{j}-\nabla \cdot \mathcal{Y}_{j}, \quad j \in \mathcal{J} ; \\
& \partial_{t}\left(\bar{\rho}_{\mathrm{m}} \widetilde{\boldsymbol{u}}_{\mathrm{m}}\right)+\nabla \cdot\left(\bar{\rho}_{\mathrm{m}} \widetilde{\boldsymbol{u}}_{\mathrm{m}} \otimes \widetilde{\boldsymbol{u}}_{\mathrm{m}}+\bar{\rho}_{\mathrm{m}} \widetilde{\mathbb{T}}_{\mathrm{r}}\right)+\nabla \bar{p} \\
& =\nabla \cdot \widetilde{\mathbb{T}}+\sum_{j \in \mathcal{J}} \widetilde{S}_{j} \widetilde{\boldsymbol{u}}_{j}+\bar{\rho}_{\mathrm{m}} \boldsymbol{g}-\nabla \cdot \mathbb{B} \\
& \partial_{t}\left(\bar{\rho}_{\mathrm{m}} \widetilde{h}_{\mathrm{m}}\right)+\nabla \cdot\left[\bar{\rho}_{\mathrm{m}}\left(\widetilde{\boldsymbol{u}}_{\mathrm{m}}+\widetilde{\boldsymbol{v}}_{h}\right) \widetilde{h}_{\mathrm{m}}\right]=\partial_{t} \bar{p}-\partial_{t}\left(\bar{\rho}_{\mathrm{m}} \widetilde{K}_{\mathrm{m}}\right) \\
& -\nabla \cdot\left[\bar{\rho}_{\mathrm{m}}\left(\widetilde{\boldsymbol{u}}_{\mathrm{m}}+\widetilde{\boldsymbol{v}}_{K}\right) \widetilde{K}_{\mathrm{m}}\right] \nabla \cdot\left(\widetilde{\mathbb{T}} \cdot \widetilde{\boldsymbol{u}}_{\mathrm{g}}-\widetilde{\boldsymbol{q}}\right)+\bar{\rho}_{\mathrm{m}}\left(\boldsymbol{g} \cdot \widetilde{\boldsymbol{u}}_{\mathrm{m}}\right) \\
& +\sum_{j \in \mathcal{J}} \widetilde{S}_{j}\left(\widetilde{h}_{j}+\widetilde{K}_{j}\right)-\nabla \cdot\left(\mathcal{Q}+\mathcal{Q}_{K}\right) .
\end{aligned}
$$


The terms $\mathcal{Y}, \mathbb{B}, \mathcal{Q}$ represent the contribution of the subgrid turbulent scales (SGS). They must be modeled to close the system in terms of the resolved fields. In ASHEE, they are

$$
\begin{aligned}
& \mathcal{Y}_{i}=\bar{\rho}_{\mathrm{m}}\left(\widetilde{y_{i} \boldsymbol{u}_{\mathrm{g}}}-\widetilde{y}_{i} \widetilde{\boldsymbol{u}}_{\mathrm{g}}\right)=-\frac{\mu_{\mathrm{t}}}{P r_{\mathrm{t}}} \nabla \widetilde{y}_{i} \\
& \mathcal{Y}_{j}=\bar{\rho}_{\mathrm{m}}\left(\widetilde{y_{j} \boldsymbol{u}_{j}}-\widetilde{y}_{i} \widetilde{\boldsymbol{u}}_{j}\right)=-\frac{\mu_{\mathrm{t}}}{P r_{\mathrm{t}}} \nabla \widetilde{y}_{j} \\
& \mathbb{B}=\bar{\rho}_{\mathrm{m}}\left(\widetilde{\boldsymbol{u}_{\mathrm{m}} \otimes \boldsymbol{u}_{\mathrm{m}}}-\widetilde{\boldsymbol{u}}_{\mathrm{m}} \otimes \widetilde{\boldsymbol{u}}_{\mathrm{m}}\right)=\frac{2}{d} \bar{\rho}_{\mathrm{m}} K_{\mathrm{t}} \mathbb{I}-2 \mu_{\mathrm{t}} \widetilde{\mathbb{S}}_{\mathrm{m}} \\
& \mathcal{Q}=\bar{\rho}_{\mathrm{m}}\left(\widetilde{h_{\mathrm{m}} \boldsymbol{u}_{\mathrm{m}}}-\widetilde{h}_{\mathrm{m}} \widetilde{\boldsymbol{u}}_{\mathrm{m}}\right)=-\frac{\mu_{\mathrm{t}}}{P r_{\mathrm{t}}} \nabla \widetilde{h}_{\mathrm{m}} \\
& \mathcal{Q}_{K}=\bar{\rho}_{\mathrm{m}}\left(\widetilde{K_{\mathrm{m}} \boldsymbol{u}_{\mathrm{m}}}-\widetilde{K}_{\mathrm{m}} \widetilde{\boldsymbol{u}}_{\mathrm{m}}\right)=-\frac{\mu_{\mathrm{t}}}{P r_{\mathrm{t}}} \nabla \widetilde{K}_{\mathrm{m}},
\end{aligned}
$$

respectively: the subgrid eddy diffusivity vector of the $i$ th phase; of the $j$ th phase; the subgrid-scale stress tensor; the diffusivity vector of the enthalpy and kinetic energy. The rate-of-shear tensor is $\widetilde{\mathbb{S}}_{\mathrm{m}}=\operatorname{sym}\left(\nabla \widetilde{\boldsymbol{u}}_{\mathrm{m}}\right)-\frac{1}{3} \nabla \cdot \widetilde{\boldsymbol{u}}_{\mathrm{m}} \mathbb{I}$. In order to close the system, the SGS viscosity $\mu_{\mathrm{t}}$, the SGS kinetic energy $K_{\mathrm{t}}$ and the SGS Prandtl number $P r_{\mathrm{t}}$ must be expressed in terms of the resolved variables, as detailed in Appendix D2.

These coefficients can be computed either statically or dynamically (see Moin et al., 1991; Bardina et al., 1980; Germano et al., 1991). In ASHEE, we implemented several SGS models (Cerminara, 2016). Currently, the code offers the possibility of choosing between: (1) the compressible Smagorinsky model, both static and dynamic (see Fureby, 1996; Yoshizawa, 1993; Pope, 2000; Chai and Mahesh, 2012; Garnier et al., 2009), (2) the subgrid-scale K-equation model, both static and dynamic (see Chacón-Rebollo and Lewandowski, 2013; Fureby, 1996; Yoshizawa, 1993; Chai and Mahesh, 2012), (3) the dynamical Smagorinsky model in the form used by Moin et al. (1991), (4) the WALE model, both static and dynamic (see Nicoud and Ducros, 1999; Lodato et al., 2009; Piscaglia et al., 2013).

Throughout this paper, we present results obtained with the dynamic WALE model (see Fig. 5 and the corresponding section for a study on the accuracy of this LES model). A detailed analysis of the influence of subgrid-scale models to simulation results is beyond the scope of this paper and will be addressed in future works (Cerminara et al., 2016).

\section{Numerical solver}

The Eulerian model described in Sect. 2, is solved numerically to obtain a time-dependent description of all independent flow fields in a three-dimensional domain with prescribed initial and boundary conditions. We have chosen to adopt an open-source approach to the code development in order to guarantee control on the numerical solution procedure and to share scientific knowledge. We hope that this will help in building a wider computational volcanology community. As a platform for developing our solver, we have chosen the unstructured, finite volume (FV) method, open-source $\mathrm{C}++$ library, OpenFOAM ${ }^{\circledR}$ (version 2.1.1). OpenFOAM ${ }^{\circledR}$, released under the Gnu Public License (GPL), has gained a vast popularity in recent years. The readily existing solvers and tutorials provide a quick start to using the code and also to inexperienced users. Thanks to a high level of abstraction in the programming of the source code, the existing solvers can be freely and easily modified in order to create new solvers (e.g., to solve a different set of equations) and/or to implement new numerical schemes. OpenFOAM ${ }^{\circledR}$ is well integrated with advanced tools for pre-processing (including meshing) and post-processing (including visualization). The support of the OpenCFD Ltd, of the OpenFOAM ${ }^{\circledR}$ foundation and of a wide developers and users community guarantees ease of implementation, maintenance and extension, suited for satisfying the needs of both volcanology researchers and of potential users - e.g., in volcano observatories. Finally, all solvers can be run in parallel on distributed memory architectures, which makes OpenFOAM ${ }^{\circledR}$ suited for envisaging large-scale, three-dimensional volcanological problems.

The new computational model, called ASHEE (the ASH Equilibrium Eulerian model) is documented in the VMSG (Volcano Modeling and Simulation Gateway) at Istituto Nazionale di Geofisica e Vulcanologia (http://vmsg.pi.ingv. it) and is made available through the VHub portal (https: //vhub.org).

\subsection{Finite volume discretization strategy}

In the FV method (Ferziger and Perić, 1996), the governing partial differential equations are integrated over a computational cell, and the Gauss theorem is applied to convert the volume integrals into surface integrals, involving surface fluxes. Reconstruction of scalar and vector fields (which are defined in the cell centroid) on the cell interface is a key step in the FV method, controlling both the accuracy and the stability properties of the numerical method.

OpenFOAM ${ }^{\circledR}$ implements a wide choice of discretization schemes. In all our test cases, the temporal discretization is based on the second-order Crank-Nicolson scheme (Ferziger and Perić, 1996), with a blending factor of 0.5 (0 meaning a first-order Euler scheme, 1 a second-order, bounded implicit scheme) and an adaptive time stepping based on the maximum initial residual of the previous time step (Kay et al., 2010), and on a threshold that depends on the Courant number $(C o<0.2)$. All advection terms of the model are treated implicitly to enforce stability. Diffusion terms are also discretized implicit in time, with the exception of those representing subgrid turbulence. The pressure and gravity terms in the momentum equations and the continuity equations are solved explicitly. However, as discussed below, the PISO (Pressure Implicit with Splitting of Operators; Issa, 1986) 
solution procedure based on a pressure correction algorithm makes such a coupling implicit. Similarly, the pressure advection terms in the enthalpy equation and the relative velocity $\boldsymbol{v}_{j}$ are made implicit when the PIMPLE (mixed SIMPLE and PISO algorithm, Ferziger and Perić, 1996) procedure is adopted. The same PIMPLE scheme is applied treating all source terms and the additional terms deriving from the equilibrium-Eulerian expansion.

In all described test cases, the spatial gradients are discretized by adopting an unlimited centered linear scheme (which is second-order accurate and has low numerical diffusion - Ferziger and Perić, 1996). Analogously, implicit advective fluxes at the control volume interfaces are reconstructed by using a centered linear interpolation scheme (also second-order accurate). The only exception is for pressure fluxes in the pressure correction equation, for which we adopt a TVD (Total Variation Diminishing) limited linear scheme (in the subsonic regimes) to enforce stability and non-oscillatory behavior of the solution. To enforce stability, the PISO loop in OpenFOAM ${ }^{\circledR}$ usually has incorporated a term of artificial diffusion for the advection term $\nabla \cdot(\rho \boldsymbol{u} \otimes$ $\boldsymbol{u})$. As studied and suggested in Vuorinen et al. (2014), we avoid using this extra term which is not present in the original PISO implementation. We refer to Jasak (1996) for a complete description of the discretization strategy adopted in OpenFOAM $^{\circledR}$.

\subsection{Solution procedure}

Instead of solving the set of algebraic equations deriving from the discretization procedure as a whole, most of the existing solvers in OpenFOAM ${ }^{\circledR}$ are based on a segregated solution strategy, in which partial differential equations are solved sequentially and their coupling is resolved by iterating the solution procedure. In particular, for Eulerian fluid equations, the momentum and continuity equation (coupled through the pressure gradient term and the gas equation of state) are solved by adopting the PISO algorithm. The PISO algorithm consists of one predictor step, where an intermediate velocity field is solved using pressure from the previous time step, and of a number of PISO corrector steps, where intermediate and final velocity and pressure fields are obtained iteratively. The number of corrector steps used affects the solution accuracy and usually at least two steps are used. Additionally, coupling of the energy (or enthalpy) equation can be achieved in OpenFOAM ${ }^{\circledR}$ through additional PIMPLE iterations (which derives from the SIMPLE algorithm by Patankar, 1980). For each transport equation, the linearized system deriving from the implicit treatment of the advectiondiffusion terms is solved by using the PbiCG solver (Preconditioned bi-Conjugate Gradient solver for asymmetric matrices) and the PCG (Preconditioned Conjugate Gradient solver for symmetric matrices), respectively, preconditioned by a Diagonal Incomplete Lower Upper decomposition (DILU) and a Diagonal Incomplete Cholesky (DIC) de- composition. The segregated system is iteratively solved until a global tolerance threshold $\epsilon_{\text {PIMPLE }}$ is achieved. In our simulations, we typically use $\epsilon_{\text {PIMPLE }}<10^{-7}$ for this threshold.

The numerical solution algorithm is designed as follows:

1. Solve the (explicit) continuity Eq. (23a) for mixture density $\rho_{\mathrm{m}}$ (predictor stage: uses fluxes from previous iteration).

2. Solve the (implicit) transport equation for all gaseous and particulate mass fractions: $y_{i}, i=1, \ldots, I$ and $y_{j}, j=1, \ldots, J$.

3. Solve the (semi-implicit) momentum equation to obtain $\boldsymbol{u}_{\mathrm{m}}$ (predictor stage: uses the pressure field from previous iteration).

4. Solve the (semi-implicit) enthalpy equation to update the temperature field $T$, the compressibility $\rho_{\mathrm{m}} / p$ (pressure from previous iteration) and transport coefficients.

5. Solve the (implicit) pressure equation and the relative velocities $\boldsymbol{v}_{j}$ to update the fluxes $\rho \boldsymbol{u}$.

6. Correct density, velocity with the new pressure field (keeping $T$ and $\rho_{\mathrm{m}} / p$ fixed).

7. Iterate from 5 evaluating the continuity error as the difference between the kinematic and thermodynamic calculation of the density (PISO loop).

8. Compute LES subgrid terms to update subgrid transport coefficients.

9. Evaluate the numerical error $\epsilon_{\mathrm{PIMPLE}}$ and iterate from 2 if prescribed (PIMPLE loop).

With respect to the standard solvers implemented in OpenFOAM $^{\circledR}$ (v2.1.1) for compressible fluid flows (e.g., sonicFoam or rhoPimpleFoam), the main modifications required are the following:

1. The mixture density and velocity replaces the fluid ones.

2. A new scalar transport equation is introduced for the mass fraction of each particulate and gas species.

3. The equations of state are modified as described in Eq. (11).

4. First-order terms from the equilibrium-Eulerian model are added in the mass, momentum and enthalpy equations.

5. Equations are added to compute flow acceleration and velocity disequilibrium.

6. Gravity terms and ambient fluid stratification are added.

7. New SGS models are implemented. 


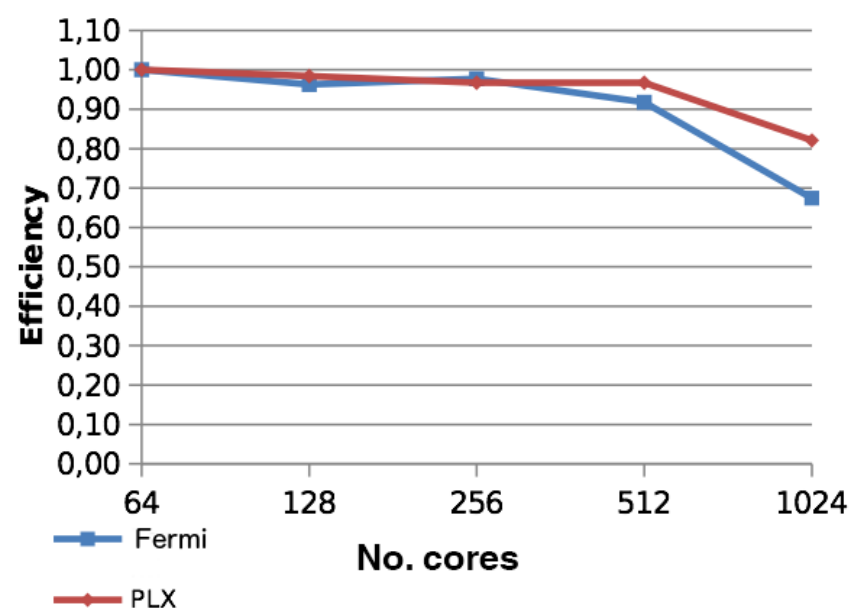

Figure 1. ASHEE parallel efficiency on Fermi and PLX supercomputers at CINECA (www.cineca.it).

Concerning point 5 , it is worth remarking that, according to Ferry et al. (2003), the first-order term $\tau_{j}$ in Eq. (15) must be limited to avoid the divergence of preferential concentration in a turbulent flow field (and to keep the effective Stokes number below 0.2). In other words, we impose at each time step that $\left|\boldsymbol{v}_{j}-\boldsymbol{w}_{j}\right| \leq 0.2\left|\boldsymbol{u}_{\mathrm{g}}+\boldsymbol{w}_{j}\right|$. We test the effect of this limiter on preferential concentration in Sect. 4.2.

\subsection{Parallel performances}

Figure 1 reports the parallel efficiency of the numerical tests described in Sect. 4.1, on both the Fermi and the PLX (a Linux cluster based on Intel Xeon ESA- and quad-core processors@2.4 GHz) machines at CINECA. The ASHEE code efficiency is very good (above 0.9 ) up to 512 cores (i.e., up to about 30000 cells core $^{-1}$ ), but it is overall satisfactory for 1024 cores, with efficiency larger than 0.8 on PLX and slightly lower (about 0.7 ) on Fermi probably due to the limited level of cache optimization and input/output scalability (Culpo, 2011). The code was run also on 2048 cores on Fermi with parallel efficiency of 0.45 (Dagna, 2013).

\section{Model verification and evaluation}

Evaluation tests are focused on the dynamics of gas (Sect. 4.1) and multiphase (Sect. 4.2) turbulence and on the mixing properties of buoyant plumes (Sect. 4.3). Compressibility likely exerts a controlling role to the near-vent dynamics during explosive eruptions (e.g., Carcano et al., 2013). Although this is not the focus of this work, we briefly discuss in Sect. 4.4 the performance of the model on a standard one-dimensional shock wave numerical test.

\subsection{Compressible decaying homogeneous and isotropic turbulence}

Turbulence is a key process controlling the dynamics of volcanic plumes since it controls the rate of mixing and air entrainment. To assess the capability of the developed model to resolve turbulence (which requires low numerical diffusion and controlled numerical errors; Geurts and Fröhlich, 2002), we have tested the numerical algorithm against different configurations of decaying homogeneous and isotropic turbulence (DHIT).

In this configuration, the flow is initialized in a domain $\Omega$ which is a box with side $L=2 \pi$ with periodic boundary conditions. As described in Blaisdell et al. (1991), Honein and Moin (2004), Pirozzoli and Grasso (2004), Lesieur et al. (2005) and Liao et al. (2009), we chose the initial velocity field so that its energy spectrum is

$\mathcal{E}(k)=\frac{16}{3} \sqrt{\frac{2}{\pi}} \frac{u_{\mathrm{rms}}}{k_{0}}\left(\frac{k}{k_{0}}\right)^{4} e^{-\frac{2 k^{2}}{k_{0}^{2}}}$,

with peak initially in $k=k_{0}$ and so that the initial kinetic energy and enstrophy are

$$
\begin{aligned}
K_{0} & =\int_{0}^{\infty} \mathcal{E}(k) \mathrm{d} k=\frac{1}{2} u_{\mathrm{rms}}^{2} \\
\mathcal{H}_{0} & =\int_{0}^{\infty} k^{2} \mathcal{E}(k) \mathrm{d} k=\frac{5}{8} u_{\mathrm{rms}}^{2} k_{0}^{2} .
\end{aligned}
$$

As reviewed by Pope (2000), the Taylor microscale can be written as a function of the dissipation $\varepsilon=2 \nu \mathcal{H}$ :

$\lambda_{\mathrm{T}}^{2} \equiv \frac{5 v u_{\mathrm{rms}}^{2}}{\varepsilon}=\frac{5 K}{\mathcal{H}}$,

thus in our configuration, the initial Taylor microscale is

$\lambda_{\mathrm{T}, 0}=\sqrt{\frac{5 K_{0}}{\mathcal{H}_{0}}}=\frac{2}{k_{0}}$.

We have chosen the non-dimensionalization keeping the root mean square of the magnitude of velocity fluctuations $\left(\boldsymbol{u}^{\prime}\right)$ equal to $u_{\mathrm{rms}}$ :

$u_{\mathrm{rms}} \equiv\left\langle\sqrt{\boldsymbol{u}^{\prime} \cdot \boldsymbol{u}^{\prime}}\right\rangle_{\Omega} \equiv \frac{1}{(2 \pi)^{3}} \int_{\Omega} \sqrt{\boldsymbol{u}^{\prime} \cdot \boldsymbol{u}^{\prime}} \mathrm{d} \boldsymbol{x}=2 \int_{0}^{\infty} \mathcal{E}(k) \mathrm{d} k$.

We also chose to make the system dimensionless by fixing $\rho_{\mathrm{m}, 0}=1, T_{0}=1, \operatorname{Pr}=1$, so that the ideal gas law becomes

$p=\rho_{\mathrm{m}} R_{\mathrm{m}} T=R_{\mathrm{m}}$,

and the initial Mach number of the mixture based on the velocity fluctuations reads

$M a_{\mathrm{rms}}=\sqrt{\frac{u_{\mathrm{rms}}^{2}}{c_{\mathrm{m}}^{2}}}=\sqrt{\frac{2 K_{0} \rho_{\mathrm{m}}}{\gamma_{\mathrm{m}} p}}=u_{\mathrm{rms}}\left(\gamma_{\mathrm{m}} p\right)^{-\frac{1}{2}}$. 
This means that $M a_{\mathrm{rms}}$ can be modified keeping fixed $u_{\mathrm{rms}}$ and modifying $p$. Following Honein and Moin (2004), we define the eddy turnover time:

$\tau_{\mathrm{e}}=\frac{\sqrt{3} \lambda_{\mathrm{T}}}{u_{\mathrm{rms}}}$.

The initial compressibility ratio $\mathrm{C}_{0}$ is defined as the ratio between the kinetic energy and its compressible component $K_{\mathrm{c}}$ :

$\mathrm{C}_{0}=\frac{K_{\mathrm{c}, 0}}{K_{0}}=\frac{1}{2(2 \pi)^{3} K_{0}} \int_{\Omega} \sqrt{\boldsymbol{u}_{\mathrm{c}}^{\prime} \cdot \boldsymbol{u}_{\mathrm{c}}^{\prime}} \mathrm{d} \boldsymbol{x}$.

Here, $\boldsymbol{u}_{\mathrm{c}}^{\prime}$ is the compressible part of the velocity fluctuations, so that $\nabla \cdot \boldsymbol{u}^{\prime}=\nabla \cdot \boldsymbol{u}_{\mathrm{c}}^{\prime}$ and $\nabla \wedge \boldsymbol{u}_{\mathrm{c}}^{\prime}=0$.

The last parameter - i.e., the dynamical viscosity - can be given by fixing the Reynolds number based either on $\lambda_{\mathrm{T}, 0}$ or $k_{0}$ :

$R e_{\lambda}=\frac{\rho_{\mathrm{m}} u_{\mathrm{rms}} \lambda_{\mathrm{T}, 0}}{\sqrt{3} \mu}$

$R e_{k_{0}}=\frac{\rho_{\mathrm{m}} u_{\mathrm{rms}}}{k_{0} \mu}$.

It is useful to define the maximum resolved wavenumber on the selected $N$-cells grid and the Kolmogorov length scale based on $R e_{k_{0}}$. They $R e_{k_{0}}$ - respectively,

$k_{\max }=\left(\frac{N}{2}-1\right) \frac{2 \pi}{L} \frac{N}{N-1}$,

$\eta=\frac{2 \pi}{k_{0}} \operatorname{Re}_{k_{0}}^{-\frac{3}{4}}$.

In order to have a Direct Numerical Simulation (DNS), the smallest spatial scale $\delta$ should be chosen in order to have $k_{\max } \eta>2$ (Pirozzoli and Grasso, 2004).

We compare the DNS of compressible decaying homogeneous and isotropic turbulence with a reference, welltested numerical solver for DNSs of compressible turbulence by Pirozzoli and Grasso (2004) and Bernardini and Pirozzoli (2009). For this comparison we fix the following initial parameters: $p=R_{\mathrm{m}}=1, \gamma_{\mathrm{m}}=1.4, P r=1, M a_{\mathrm{rms}}=0.2$, $\mathrm{C}_{0}=0, u_{\mathrm{rms}}^{2}=2 K_{0}=0.056, k_{0}=4, \lambda_{\mathrm{T}}=0.5, \tau_{\mathrm{e}} \simeq 3.6596$, $\mu=5.885846 \times 10^{-4}, R e_{\lambda} \simeq 116, R e_{k_{0}} \simeq 100$. Thus a grid with $N=256^{3}$ cells gives $k_{\max } \simeq 127$ and $k_{\max } \eta=2 \pi$, big enough to have a DNS. The simulation has been performed on 1024 cores on the Fermi Blue Gene/Q infrastructure at Italian CINECA super-computing center (http://www.cineca. it), on which about $5 \mathrm{~h}$ are needed to complete the highestresolution runs $\left(256^{3}\right.$ cells) up to time $t / \tau_{\mathrm{e}}=5.465$ (about 3500 time steps). The average computing speed on 1024 Fermi cores is about 1-3 Mcells/s, with the variability associated with the number of solid phases described by the model. This value is confirmed in all benchmark cases presented in this paper.

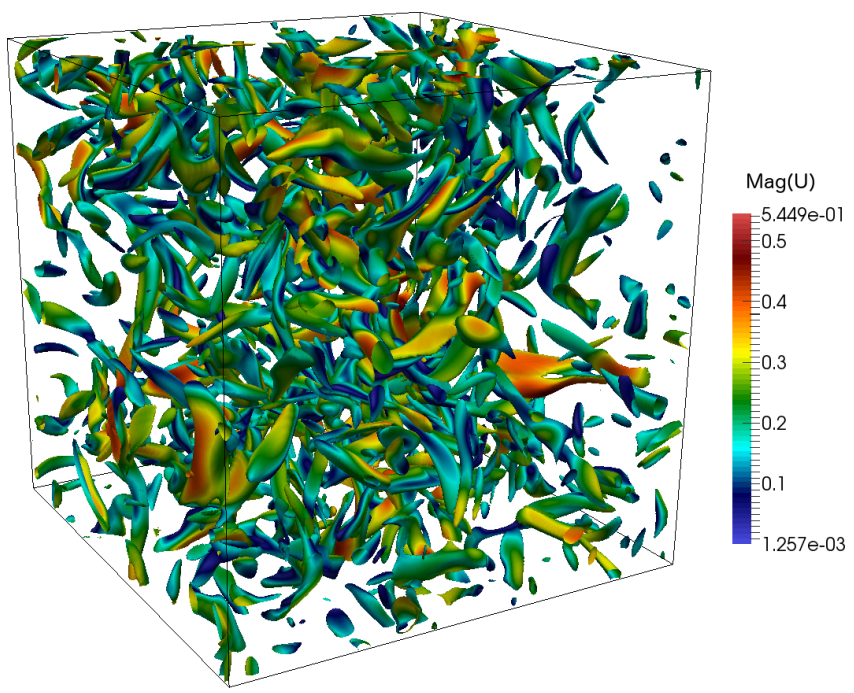

Figure 2. Isosurface at $Q_{u} \simeq 19 \mathrm{~Hz}^{2}$ and $t / \tau_{\mathrm{e}} \simeq 2.2$, representing zones with coherent vortices.

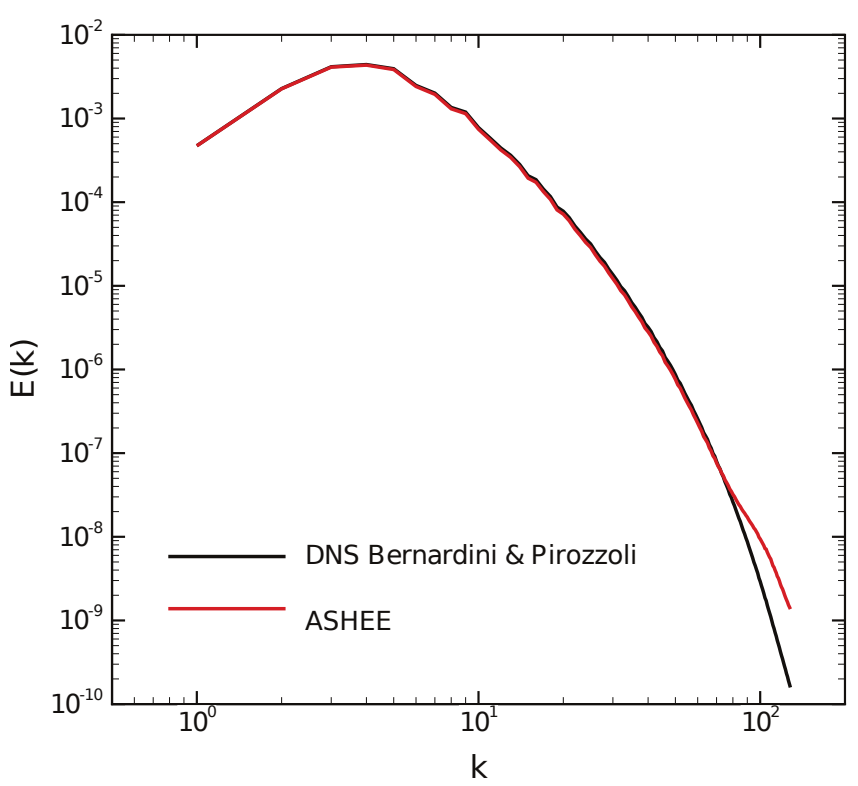

Figure 3. Comparison of a DNS executed with the eight order scheme by Pirozzoli and Grasso (2004) and our code implemented using the $\mathrm{C}++$ libraries of OpenFOAM ${ }^{\circledR}$ at $t / \tau_{\mathrm{e}}=1.093$. The $L^{2}$ norm between the two spectra is $4.0 \times 10^{-4}$. The main parameters are $R e_{\lambda} \simeq 116, M a_{\mathrm{rms}}=0.2$.

Figure 2 shows an isosurface of the second invariant of the velocity gradient, defined as

$Q_{u}=\frac{1}{2}\left((\operatorname{Tr}(\nabla \boldsymbol{u}))^{2}-\operatorname{Tr}(\nabla \boldsymbol{u} \cdot \nabla \boldsymbol{u})\right)$.

The so-called $Q$-criterion (Garnier et al., 2009) allows the identification of coherent vortices inside a three dimensional velocity field. 


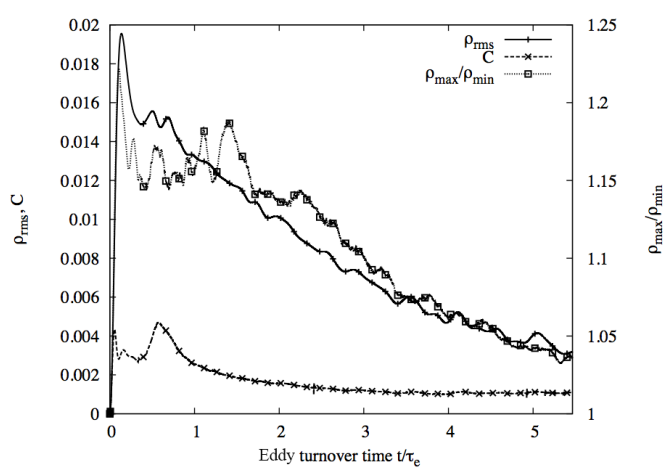

(a)

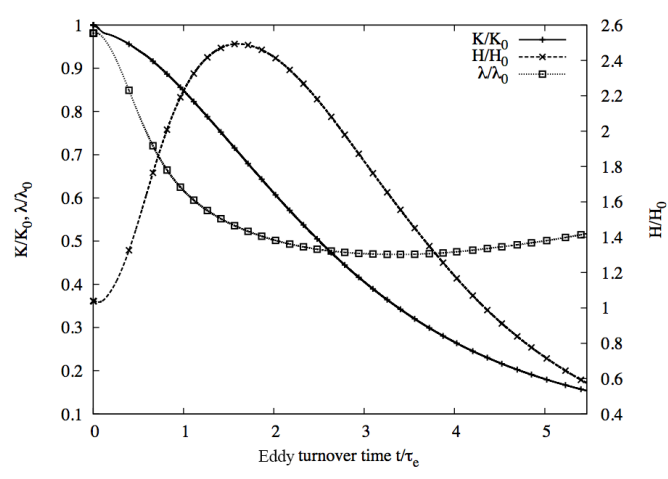

(c)

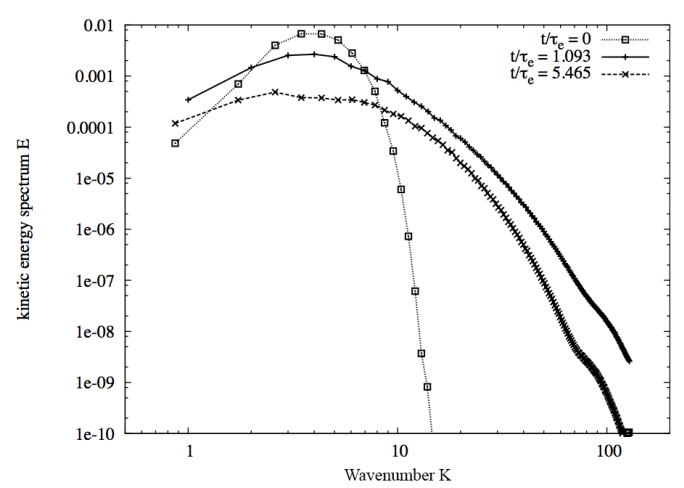

(b)

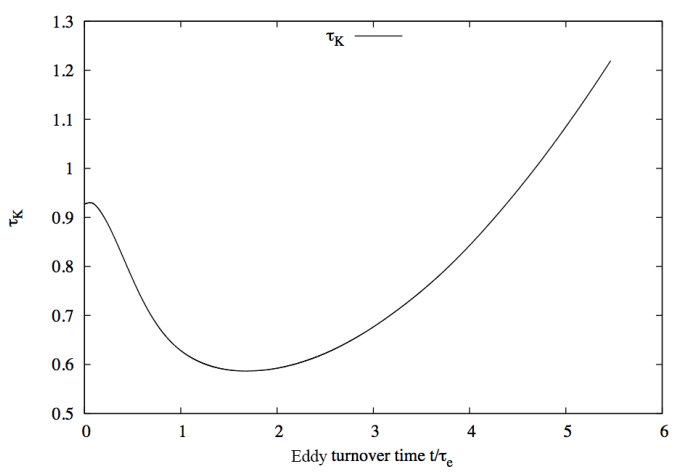

(d)

Figure 4. Evolution of dynamical quantities in DHIT with $R e_{\lambda} \simeq 116$ and $M a_{\mathrm{rms}}=0.2$ at $t / \tau_{\mathrm{e}}=5.465$. (a) Density fluctuations $\rho_{\mathrm{rms}}$, compressibility $\mathrm{C}$ and density contrast $\rho_{\max } / \rho_{\min } ;(\mathbf{b})$ evolution of the energy spectrum $\mathcal{E}(k)$; (c) non-dimensional kinetic energy $K / K_{0}$, enstrophy $\mathcal{H} / \mathcal{H}_{0}$ and Taylor microscale $\lambda_{\mathrm{T}} / \lambda_{\mathrm{T}, 0} ;(\mathbf{d})$ Kolmogorov timescale $\tau_{\eta}$.

In Fig. 3 we present a comparison of the energy spectrum $\mathcal{E}(k)$ obtained with the ASHEE model and the model by Bernardini and Pirozzoli (2009) after approximatively 1 eddy turnover time; the $L^{2}$ norm of the difference between the two spectra is $4.0 \times 10^{-4}$. This validates the accuracy of our numerical code in the single-phase and shock-free case.

Figure 4 shows the evolution of several integral parameters describing the dynamics of the decaying homogeneous and isotropic turbulence. Figure 4a displays the density fluctuations $\rho_{\mathrm{rms}}=\sqrt{\left\langle\left(\rho-\langle\rho\rangle_{\Omega}\right)^{2}\right\rangle_{\Omega}}$, the density contrast $\rho_{\max } / \rho_{\min }$ and the standard measure of compressibility $\mathrm{C}=\left\langle|\nabla \cdot \boldsymbol{u}|^{2}\right\rangle_{\Omega} /\left\langle|\nabla \boldsymbol{u}|^{2}\right\rangle_{\Omega}$ which takes value between 0 (incompressible flow) and 1 (potential flow) (Boffetta et al., 2007). All the quantities shown in Fig. 4 a depend on the initial Mach number and compressibility. For the case shown, $M a_{\mathrm{rms}}=0.2$ and we obtain very similar results to those reported in Figs. 18 and 19 by Garnier et al. (1999).

Figure $4 \mathrm{~b}$ shows the kinetic energy spectrum at $t / \tau_{\mathrm{e}}=$ $0,1.093,5.465$. The energy spectrum widens from the initial condition until its tail reaches $k \simeq k_{\max } \simeq 127$. Then the system dissipates and the maximum of the energy spectrum decreases. The largest scales tend to lose energy more slowly than the other scales and the spectrum widens also in the larger-scale direction.

Figure $4 \mathrm{c}$ presents the evolution of $K$ (total turbulent kinetic energy), $\mathcal{H}$ (enstrophy) and $\lambda_{\mathrm{T}}$ (Taylor microscale). The total kinetic energy decreases monotonically and at $t \simeq 5.5 \tau_{\mathrm{e}}$ just $\simeq 15 \%$ of its initial value is conserved. On the other hand, enstrophy increases until it reaches a maximum at $1.5<t / \tau_{\mathrm{e}}<2$. It then starts to decrease monotonically. This behavior is related to the two different stages we have highlighted in the analysis of the energy spectrum evolution. In the first stage, viscous effects are negligible and enstrophy increases due to vortex stretching. During the second stage, viscous diffusion starts to have an important role and distorted dissipative structures are created (Garnier et al., 1999). Also the Taylor microscale reflects this behavior, reaching a minimum at the end of the first stage and increasing monotonically during the second stage of the evolution. This is a characteristic of the magnitude of the velocity gradients in the inertial range: by comparing it with $\delta$ we can have an idea of the broadness of the range of wave numbers where the flow is dissipative. In this DNS, we have $\lambda_{\mathrm{T}} \simeq 10.2 \delta$ at $t \simeq 5.5 \tau_{\mathrm{e}}$. 
Table 1. Stokes time, maximum Stokes number and diameter of the solid particles inserted in the turbulent box.

\begin{tabular}{lll}
\hline$\tau_{j}$ & $S t_{\max }=\tau_{j} / 0.6$ & $d_{j}\left(\hat{\rho}_{j}=10^{3}\right)$ \\
\hline 0.60 & 1.0 & $2.521 \times 10^{-3}$ \\
0.30 & 0.5 & $1.783 \times 10^{-3}$ \\
0.15 & 0.25 & $1.261 \times 10^{-3}$ \\
0.075 & 0.125 & $8.914 \times 10^{-4}$ \\
0.0375 & 0.0625 & $6.303 \times 10^{-4}$ \\
\hline
\end{tabular}

In Fig. 4d we show the evolution of the Kolmogorov timescale $\tau_{\eta}$ during the evolution of the decaying turbulence.

We finally compare in Fig. 5 the DNS described with simulations at lower resolution with $N=32^{3}$ cells and $N=$ $64^{3}$ cells. In this case, it is expected that the spectra diverge from the DNS, unless an appropriate subgrid model is introduced to simulate the effects of the unresolved to the resolved scales. Several subgrid models have been tested (Cerminara, 2016), both static and dynamic. Figure 5 presents the resulting spectrum using the dynamic WALE model (Nicoud and Ducros, 1999; Lodato et al., 2009). In this figure, we notice how the dynamic WALE model works pretty well for both the $32^{3}$ and $64^{3}$ LES, avoiding the smallest scales to accumulate unphysical energy.

\subsection{Multiphase isotropic turbulence}

In this section we test the capability of ASHEE to correctly describe the decoupling between solid and gaseous phases when $S t_{j}<0.2$ and to explore its behavior when the equilibrium-Eulerian hypothesis $S t_{j}<0.2$ is not fulfilled so that a limiter to the relative velocity $\boldsymbol{u}_{\mathrm{g}}-\boldsymbol{u}_{j}$ is applied. We mainly refer to Rani and Balachandar (2003) for a quantitative assessment of numerical model results.

To this aim, we performed a numerical simulation of homogeneous and isotropic turbulence with a gas phase initialized with the same initial and geometric conditions described in Sect. 4.1. We added to that configuration five solid particle classes chosen in such a way that $S t_{j} \in[0.03,1]$, homogeneously distributed and with zero relative velocity: $\boldsymbol{v}_{j}(\boldsymbol{x}, 0)=0$. From Fig. 4d, we see that, during turbulence decay, approximately $\tau_{\eta} \in[0.6,1.2]$. Therefore, for a given particle class with $\tau_{j}$ fixed, during the time interval $t / \tau_{\mathrm{e}} \in$ $[0,5.5]$ we have $S t_{\max } / S t_{\min } \simeq 2$. In Table 1 we report the main properties of the particles inserted in the turbulent box. To evaluate the Stokes time here we used $\tau_{j}=\hat{\rho}_{j} d_{j}^{2} /(18 \mu)$ because in the absence of settling, $R e_{j}<1$ when $S t_{j}<1$ (Balachandar, 2009). We set the material density of all the particles to $\hat{\rho}_{j}=10^{3}$. In order to have a small contribution of the particle phases to the fluid dynamics - one way coupling - here we set the solid particle mass fraction to a small value, $y_{j}=0.002$, so that $y_{\mathrm{g}}=0.99$.

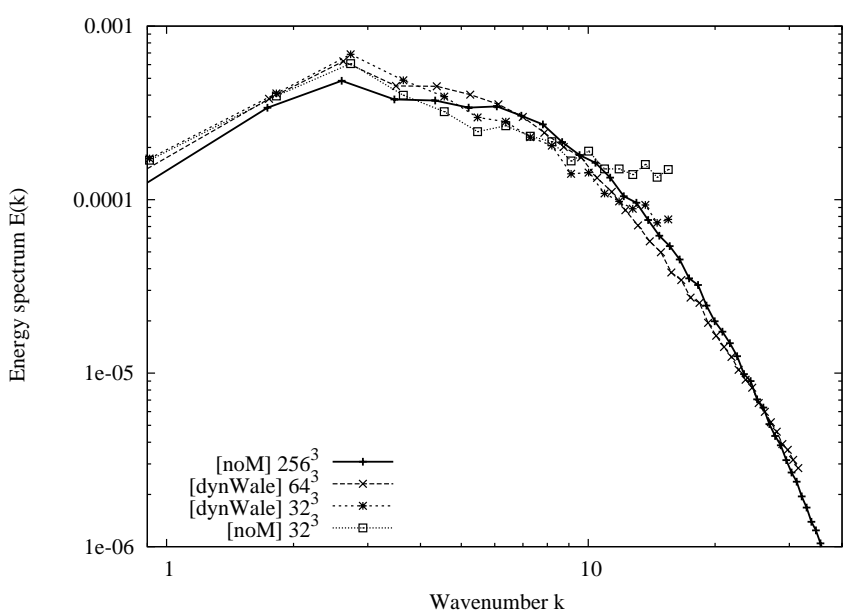

Figure 5. Energy spectrum $\mathcal{E}(k)$ at $t / \tau_{\mathrm{e}}=5.465$ obtained with different spatial resolutions and with/without subgrid-scale LES model.

In Fig. 6 we show a slice of the turbulent box at $t / \tau_{\mathrm{e}} \simeq 2.2$. Panel (a) displays the solid mass fraction, highlighting the preferential concentration and clustering of particles in response to the development of the acceleration field (panel b) associated with turbulent eddies.

As described in Maxey (1987) and Rani and Balachandar (2003), a good measure for the degree of preferential concentration in incompressible flows is the weighted average on the particle mass fraction of the quantity $\left(|\mathcal{D}|^{2}-|\mathcal{S}|^{2}\right)$, where $\mathcal{S}$ is the vorticity tensor - i.e., the skew-symmetric part of the gas velocity gradient - and $\mathcal{D}$ is its symmetrical part. For compressible flows, we choose to consider

$\langle\mathcal{P}\rangle_{j} \equiv\left\langle\left(|\mathcal{D}|^{2}-|\mathcal{S}|^{2}-|\operatorname{Tr}(\mathcal{D})|^{2}\right)\right\rangle_{j} \equiv \frac{\left\langle y_{j}\left(\mathcal{P}-\langle\mathcal{P}\rangle_{\Omega}\right)\right\rangle_{\Omega}}{\left\langle y_{j}\right\rangle_{\Omega}}$.

This is a good measure because (use integration by parts, the Gauss theorem and Eq. 15) with $\boldsymbol{w}_{j}=0$,

$$
\begin{aligned}
& \left\langle\nabla \cdot \boldsymbol{u}_{j}\right\rangle_{\Omega}=-\tau_{j}\left\langle\sum_{l, m}\left(\partial_{l} u_{m} \partial_{m} u_{l}-\partial_{l} u_{l} \partial_{m} u_{m}\right)\right\rangle_{\Omega} \\
& =-\tau_{j}\left\langle\left(|\mathcal{D}|^{2}-|\mathcal{S}|^{2}-|\operatorname{Tr}(\mathcal{D})|^{2}\right)\right\rangle_{\Omega} .
\end{aligned}
$$

Moreover, it is worth noting that $\langle\mathcal{P}\rangle_{j}$ vanishes in the absence of preferential concentration. By dimensional analysis, preferential concentration is expected to behave as

$$
\langle\mathcal{P}\rangle_{j} \propto \begin{cases}\tau_{j} / \tau_{\eta}^{3} & \mathrm{DNS} \\ \tau_{j} / \tau_{\xi}^{3} & \mathrm{LES}\end{cases}
$$

because it must be proportional to $\tau_{j}$ and have a dimension of $\left[s^{-2}\right]$. As described by Pope (2001), the typical timescale 


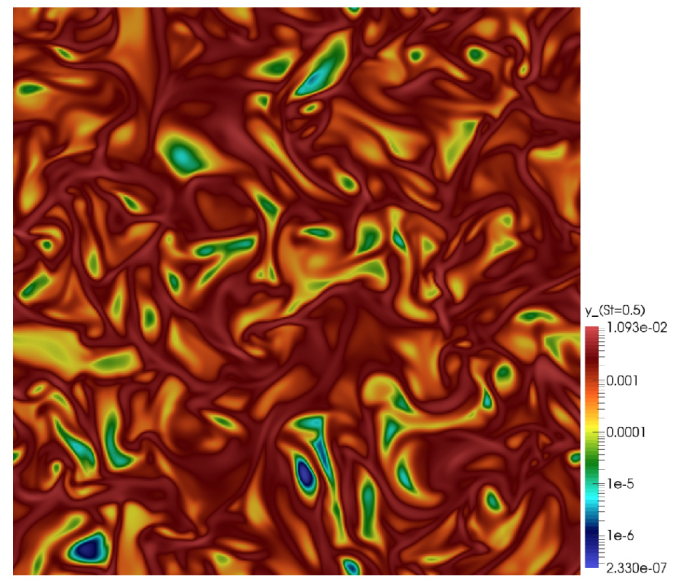

(a) Mass fraction

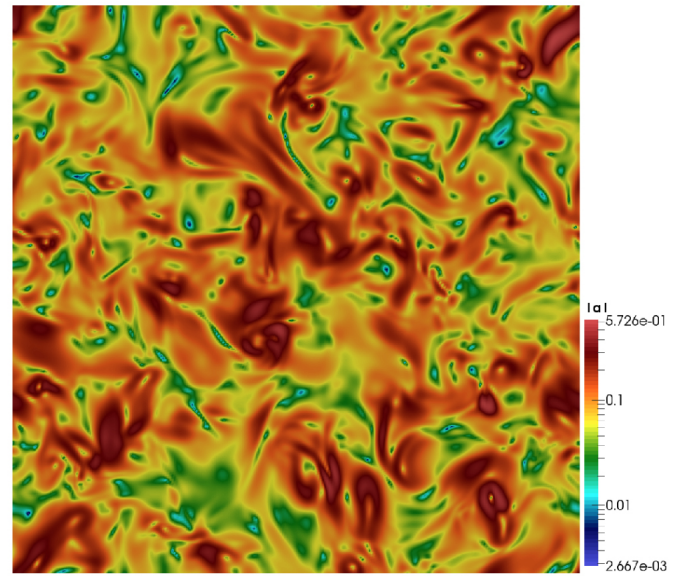

(b) Acceleration

Figure 6. Slice of the turbulent box at $t / \tau_{\mathrm{e}} \simeq 2.2$. The two panels represent respectively a logarithmic color map of $y_{3}\left(S t_{\mathrm{max}}=0.5\right)$ and of $\left|\boldsymbol{a}_{\mathrm{g}}\right|$.

corresponding to an eddy length scale $\xi$ in the inertial subrange, can be evaluated by means of the Kolmogorov's theory as

$\tau_{\xi}=\tau_{\lambda}\left(\frac{\xi}{\lambda_{\mathrm{T}}}\right)^{\frac{2}{3}}$

where the Taylor microscale $\lambda_{\mathrm{T}}$ is defined by Eq. (28). Since the time based on the Taylor microscale is defined as

$\tau_{\lambda}=\frac{\sqrt{3} \lambda_{\mathrm{T}}}{u_{\mathrm{rms}}}$

we can evaluate the typical time at the smallest resolved LES scale $\xi$ knowing the kinetic energy $K(t)$ and $\lambda_{\mathrm{T}}(t)$ :

$\tau_{\xi}(t)=\sqrt{\frac{3}{2 K(t)}} \xi^{\frac{2}{3}} \lambda_{\mathrm{T}}(t)^{\frac{1}{3}}$.

In Fig. 7 we show the time evolution of the degree of preferential concentration as a function of the Stokes number for both DNS with $256^{3}$ cells and the LES with $32^{3}$ cells. There, we multiply $\langle\mathcal{P}\rangle_{j}$ by $\tau_{\xi} \tau_{j}$ in order to make it dimensionless and to plot on the same graph all the different particles at different times together.

At $t=0$ the preferential concentration is zero for all Stokes numbers. Then, preferential concentration of each particle class increases up to a maximum value and then it decreases because of the decaying of the turbulent energy. The maximum degree of preferential concentration is reached by each particle class when $\tau_{\eta}$ is minimum (at $t / \tau_{\mathrm{e}} \simeq 1.7$, see Fig. 4d). Then, $\langle\mathcal{P}\rangle_{j}$ decreases and merges with the curve relative to the next particle class at the final simulation time, when $\tau_{\eta}$ is about twice its minimum. Note that the expected behavior of Eq. (42) is reproduced for $S t_{j}<0.2$ and in particular we find

$$
\langle\mathcal{P}\rangle_{j} \simeq \begin{cases}1.52 S t_{j} \tau_{\eta}^{-2} & \text { DNS } \\ 1.52 S t_{j} \tau_{\xi}^{-2} & \text { LES. }\end{cases}
$$

Moreover, by comparing our results with the EulerianLagrangian simulation described in Rani and Balachandar (2003), we note that our limiter for the preferential concentration when $S t>0.2$ is well behaving.

For the sake of completeness, we found that the best fit in the range $S t<2.5$ for the data found by Rani and Balachandar (2003) is

$\langle\mathcal{P}\rangle_{j} \simeq 1.52 \times \frac{S t_{j}}{1+3.1 \times S t_{j}+3.8 \times S t_{j}^{2}} \tau_{\eta}^{-2}$,

with root mean square of residuals $8.5 \times 10^{-3}$.

Regarding the $32^{3}$ LES simulation, Fig. 7 shows that the Stokes number of each particle class in the LES case is much smaller than its DNS counterpart. In accord with Balachandar and Eaton (2010), we have

$S t_{\xi}=S t_{\eta}\left(\frac{\eta}{\xi}\right)^{\frac{2}{3}}$

confirming that the equilibrium-Eulerian model widens its applicability under the LES approximation. Also note that the presented LES is able to reproduce the expected degree of preferential concentration with a satisfactory level of accuracy when $S t<0.2$. In particular, the LES slightly overestimates preferential concentration and the time needed to reach the equilibrium and to "forget" the particle initial condition. 


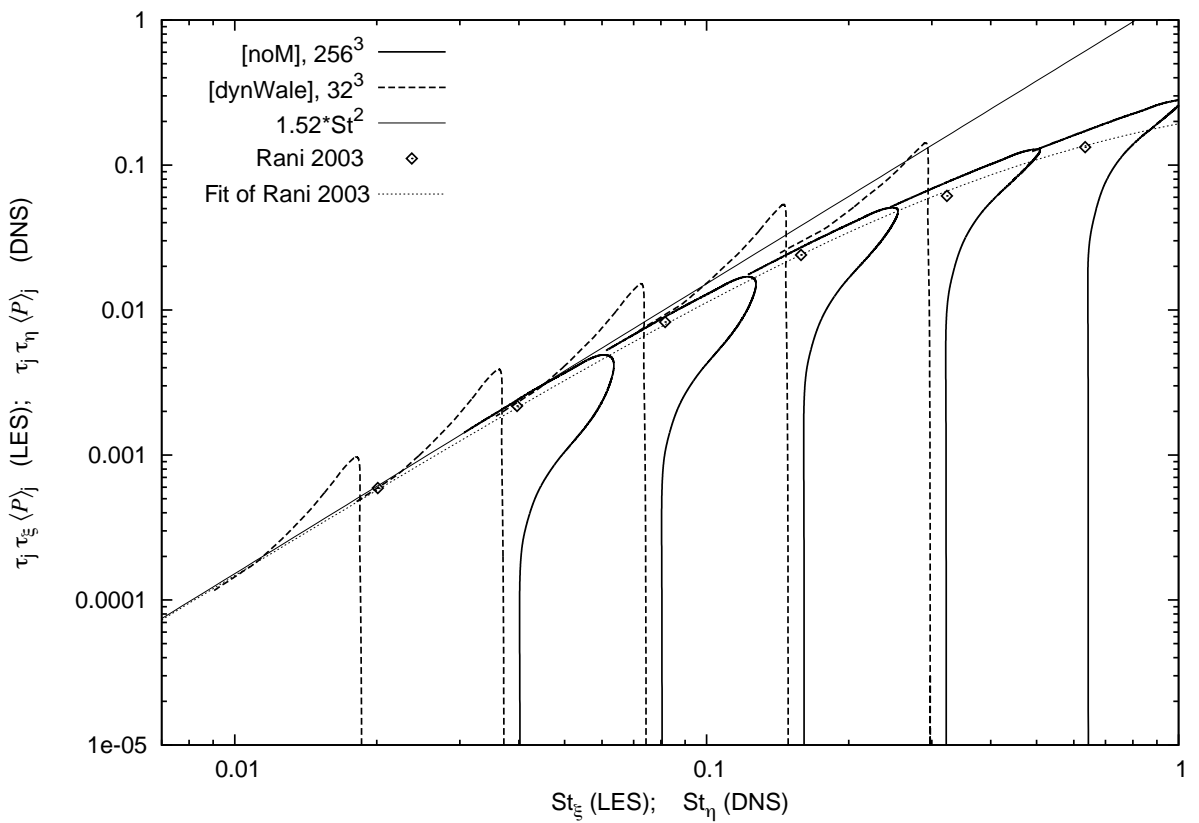

Figure 7. Evolution of the degree of preferential concentration with $S t_{\xi}$ (LES) or $S t_{\eta}$ (DNS). We obtain a good agreement between equilibrium-Eulerian LES/DNS and Lagrangian DNS simulations. The fit for the data by Rani and Balachandar (2003) is found in Eq. (47).

\subsection{Turbulent forced plume}

As a third benchmark, we discuss high-resolution, threedimensional numerical simulation of a forced gas plume, produced by the injection of a gas flow from a circular inlet into a stable atmospheric environment at lower temperature (and higher density). Such an experiment allows us to test the numerical model behavior against some of the fundamental processes controlling volcanic plumes, namely density variations, non-isotropic turbulence, mixing, air entrainment and thermal exchange. This study is mainly aimed at assessing the capability of the numerical model to describe the time-average behavior of a turbulent plume and to reproduce the magnitude of large-scale fluctuations and large-eddy structures. We will mainly refer to laboratory experiments by George et al. (1977) and Shabbir and George (1994) and numerical simulations by Zhou et al. (2001) for a quantitative assessment of model results.

Numerical simulations describe a vertical round forced plume with heated air as the injection fluid. The plume axis is aligned with the gravity vector and is subjected to a positive buoyancy force. The heat source diameter $2 b_{0}$ is $6.35 \mathrm{~cm}$, the exit vertical velocity on the axis $u_{0}$ is $0.98 \mathrm{~m} \mathrm{~s}^{-1}$, the inflow temperature $T_{0}$ is $568 \mathrm{~K}$ and the ambient air temperature $T_{\alpha}$ is $300 \mathrm{~K}$. The corresponding Reynolds number is 1273 , based on the inflow mean velocity, viscosity and diameter. Air properties at inlet are $C_{p}=1004.5 \mathrm{~J}\left(\mathrm{~K} \mathrm{~kg}^{-1}\right.$, $R=287 \mathrm{~J}(\mathrm{~K} \mathrm{~kg})^{-1}$ and $\mu=3 \times 10^{-5}$ Pas.

As discussed by Zhou et al. (2001) the development of the turbulent plume regime is quite sensitive to the inlet condi- tions: we therefore tested the model by adding a periodic perturbation and a non-homogeneous inlet profile to anticipate the symmetry breaking, and the transition from a laminar to a turbulent flow. The radial profile of vertical velocity has the form

$u(r)=\frac{1}{2} u_{0}\left[1-\tanh \left(\frac{b_{0}}{4 \delta_{r}}\left(\frac{r}{b_{0}}-\frac{b_{0}}{r}\right)\right)\right]$,

where $\delta_{r}$ is the thickness of the turbulent boundary layer at the plume inlet, that we have set at $\delta_{r}=0.1 b_{0}$. A periodical forcing and a random perturbation of intensity $0.05 u_{0}$ has been superimposed to mimic a turbulent inlet.

The resulting average mass, momentum and buoyancy flux are $Q_{0}=2.03 \times 10^{-3} \mathrm{~kg} \mathrm{~s}^{-1}, M_{0}=1.62 \times 10^{-3} \mathrm{~kg} \mathrm{~m} \mathrm{~s}^{-2}$ and $F_{0}=1.81 \times 10^{-3} \mathrm{~kg} \mathrm{~s}^{-1}$.

The computational grid is composed of $360 \times 180 \times 180$ uniformly spaced cells (deformed near the bottom plane to conform to the circular inlet) in a box of size $12.8 \times 6.4 \times 6.4$ diameters. In particular, the inlet is discretized with 400 cells. The adaptive time step was set to keep the $C o<0.2$. Based on estimates by Plourde et al. (2008), the selected mesh refinement is coarser than the required grid to fully resolve turbulent scales in a DNS (which would require about $720 \times 360 \times 360$ cells). Nonetheless, this mesh is resolved enough to avoid the use of a subgrid-scale model. This can be verified by analyzing the energy spectra of fluctuations on the plume axis and at the plume outer edges. In Fig. 8 we show the energy spectra of temperature and pressure as a function of the non-dimensional frequency: the Strouhal number Str $=f \times 2 b_{0} / u_{0}(f$ is the frequency in $[\mathrm{Hz}])$. We recover 
a result similar to Plourde et al. (2008), where the inertialconvective regime with the decay $-5 / 3$ and the inertialdiffusive regime with the steeper decay -3 are observable (List, 1982).

Model results describe the establishment of the turbulent plume through the development of fluid-dynamic instabilities near the vent (puffing is clearly recognized as a toroidal vortex in Fig. 9a). The breaking of large-eddies progressively leads to the onset of the developed turbulence regime - which is responsible for the mixing with the surrounding ambient air, radial spreading of the plume and decrease of the plume average temperature and velocity. Figure 9 a displays the spatial distribution of gas temperature. Mixing becomes effective above a distance of about four diameters. Figure $9 \mathrm{~b}$ displays the distribution of the vorticity, represented by values of the $Q_{u}$ invariant (Eq. 39). The figure clearly identifies the toroidal vortex associated with the first instability mode (puffing, dominant at such Reynolds numbers). We observed the other instability modes (helical and meandering; Lesieur et al., 2005) only by increasing the forcing intensity (not shown).

Experimental observations by George et al. (1977) and Shabbir and George (1994) reveal that the behavior of forced plumes far enough from the inlet can be well described by integral one-dimensional plume models (Morton et al., 1956; Morton, 1959) provided that an adequate empirical entrainment coefficient is used. In the buoyant plume regime at this Reynolds number George et al. (1977) obtained an entrainment coefficient of 0.153 .

To compare numerical result with experimental observations and one-dimensional average plume models, we have time-averaged the numerical results between 4 and $10 \mathrm{~s}$ (when the turbulent regime was fully developed) and computed the vertical mass $Q(z)$, momentum $M(z)$ and buoyancy $F(z)$ fluxes as a function of the height. To perform this operation, we define the time-averaging operation $(\overline{.})$ and the horizontal domain:

$$
\begin{aligned}
\mathcal{O}(z) & =\left\{(x, y) \in \mathbb{R}^{2} \mid\left(\bar{y}_{\text {tracer }}(\boldsymbol{x})>0.01\right.\right. \\
& \left.\left.\times y_{\text {tracer }, 0}\right) \wedge\left(\bar{u}_{z}(\boldsymbol{x})>0\right)\right\},
\end{aligned}
$$

where $(x, y, z)=\boldsymbol{x}$ are the spatial coordinates, $y_{\text {tracer }}$ is the mass fraction field of a tracer injected from the vent with initial mass fraction $y_{\text {tracer }, 0}$ and $u_{z}$ is the axial component of the velocity field. We use this definition for $\mathcal{O}(z)$ for coherence with integral plume models, where the mean velocity field is assumed to have the same direction as the plume axis (see Morton et al., 1956; Woods, 1988; Cerminara, 2015, 2016; Cerminara et al., 2016). This hypothesis is tested in Fig. 10a, where it can be verified that the time-averaged streamlines inside the plume are parallel to the axis (Fig. 10b shows the instantaneous streamlines and velocity magnitude field).

The plume fluxes are evaluated as follows (see George et al., 1977; Shabbir and George, 1994; Kaminski et al., 2005):
- mass flux $Q(z)=\int_{\mathcal{O}} \bar{\rho} \bar{u}_{z} \mathrm{~d} x \mathrm{~d} y$,

- momentum flux $M(z)=\int_{\mathcal{O}} \bar{\rho} \bar{u}_{z}^{2} \mathrm{~d} x \mathrm{~d} y$,

- buoyancy flux $F(z)=\int_{\mathcal{O}} \bar{u}_{z}\left(\rho_{\alpha}-\bar{\rho}\right) \mathrm{d} x \mathrm{~d} y$,

where $\rho_{\alpha}=\rho_{\alpha}(z)$ is the atmospheric density. From these quantities it is possible to retrieve the main plume parameters:

- plume radius $b(z)=\sqrt{\frac{Q(F+Q)}{\pi \rho_{\alpha} M}}$,

- plume density $\beta(z)=\rho_{\alpha} \frac{Q}{(F+Q)}$,

- plume temperature $T_{\beta}(z)=T_{\alpha} \frac{F+Q}{Q}$,

- plume velocity $U(z)=\frac{M}{Q}$,

- entrainment coefficient $\kappa(z)=\frac{Q^{\prime}}{2 \pi \rho_{\alpha} U b}$,

where $(\cdot)^{\prime}$ is the derivative along the plume axis and $T_{\alpha}$ is the atmospheric temperature profile.

Figure 11 displays the average plume radius and velocity. As previously reported by Fanneløp and Webber (2003) and Plourde et al. (2008), the plume radius initially shrinks due to the sudden increase of velocity due to buoyancy (at $z=0.1 \mathrm{~m}$ ). Above, turbulent mixing becomes effective and increases the plume radius while decreasing the average velocity. The upper inset in Fig. 11 shows the values of the vertical mass $q=Q / Q_{0}$, momentum $m=M / M_{0}$ and buoyancy $f=F / F_{0}$, normalized with the inlet values. All variables have the expected trends and, in particular, the buoyancy flux is constant (as expected for weak ambient stratification) whereas $q$ and $m$ monotonically increase and attain the theoretical asymptotic trends shown also in Fig. 12. Indeed, Fanneløp and Webber (2003) have shown that an integral plume model for non-Boussinesq regimes (i.e., large density contrasts) in the approximation of weak ambient stratification and adopting the Ricou and Spalding (1961) formulation for the entrainment coefficient, has a first integral such that $q^{2}$ is proportional to $m^{5 / 2}$ at all elevations. Figure 12 demonstrates that this relationship is well reproduced by our numerical simulations, as also observed in DNS by Plourde et al. (2008).

The lower inset in Fig. 11 shows the computed entrainment coefficient, which is very close to the value found in experiments (George et al., 1977; Shabbir and George, 1994) and numerical simulations (Zhou et al., 2001) of an analogous forced plume. We found a value around 0.14 in the buoyant plume region $\left(6.4<z / 2 b_{0}<16\right)$.

The analysis of radial profiles led to a similar conclusions: in Fig. 13, we show the evolution of the radial profiles for the mean vertical velocity field. In this figure, we also report the plume radius as evaluated from Gaussian fits of these profiles on horizontal slices: 


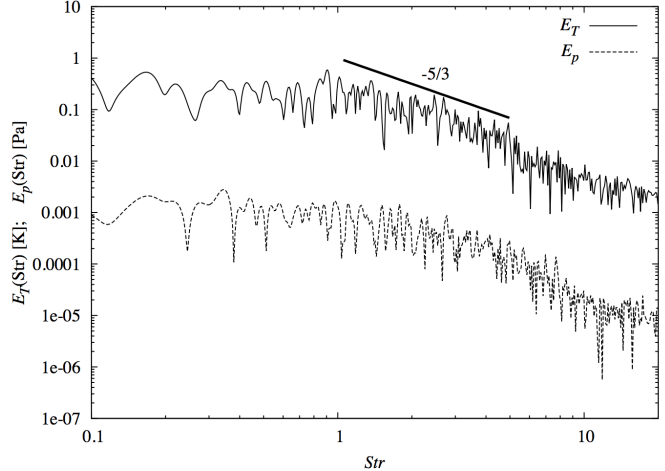

(a) Fluctuations at an axial point

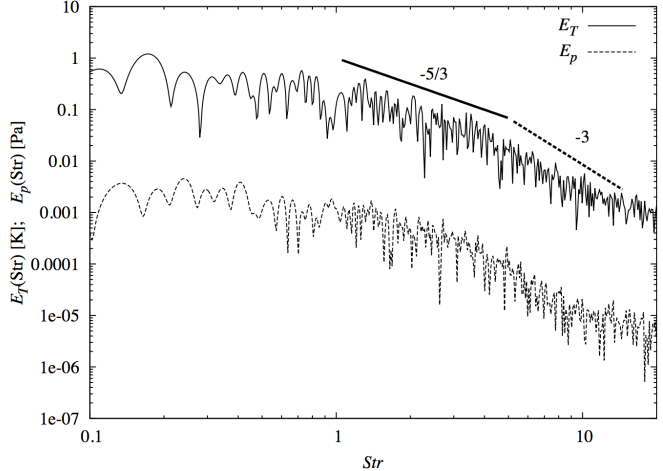

(b) Fluctuations at an outer edge point

Figure 8. Temperature (solid) and pressure (dashed) fluctuations energy spectra: (a) at a point along the plume axis $(0,0,0.5715) \mathrm{m}$; (b) at a point along the plume outer edge $(0,0.06858,0.5715) \mathrm{m}$. The slopes $\mathrm{Str}^{-5 / 3}$ and $\mathrm{Str}^{-3}$ are represented with a thick solid and dashed line respectively.
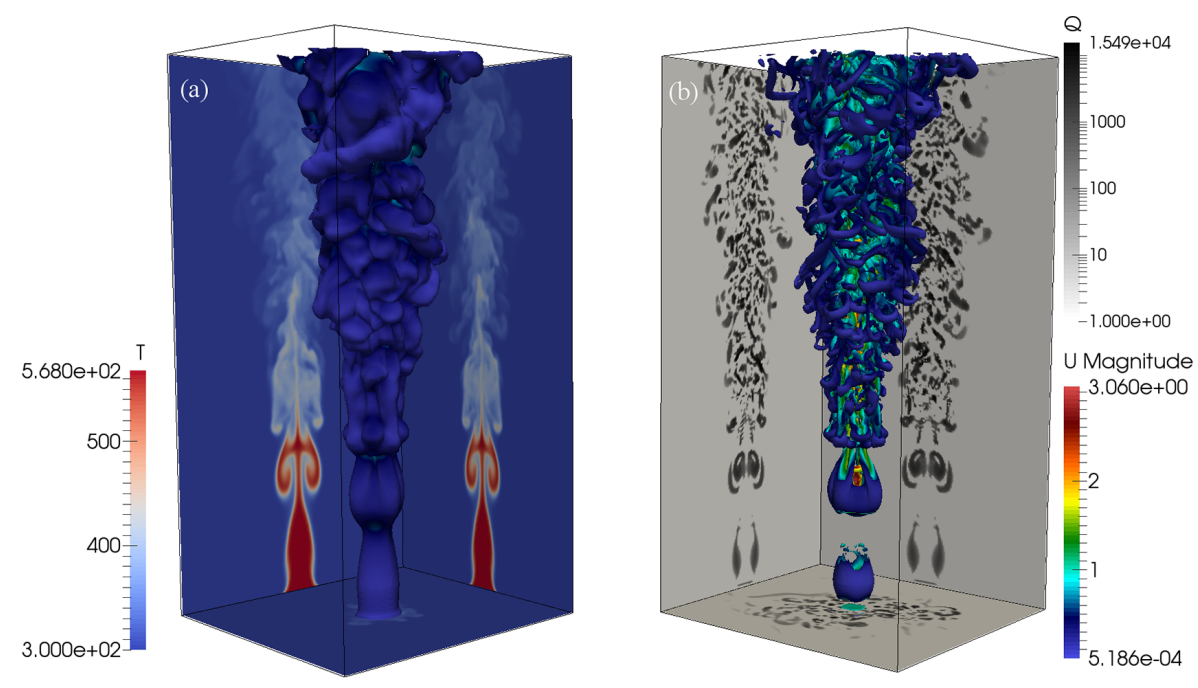

Figure 9. Three-dimensional numerical simulation of a forced gas plume at $t=10 \mathrm{~s}$. (a) Isosurface of temperature $T=305 \mathrm{~K}$, colored with the magnitude of velocity, and the temperature distribution on two orthogonal slices passing across the inlet center. (b) Isosurface of $Q_{u}=100 \mathrm{~s}^{-2}$ colored with the value of the velocity magnitude, and its distribution across two vertical slices passing through the inlet center.

$\bar{u}_{z}(x, y)=U_{\text {fit }} \exp \left(-\frac{x^{2}+y^{2}}{b_{\text {fit }}^{2}}\right)$.

The slope of the function $b_{\text {fit }}(z)$ has been evaluated in the region $6.4<z / 2 b_{0}<16$, to obtain $b_{\text {fit }} / z=0.142 \pm 0.001$ to be compared with the result of George et al. (1977): $b_{\text {fit }} / z=0.135 \pm 0.010$.

Finally, Fig. 14 reports the time-averaged values of the vertical velocity and temperature along the plume axis. As observed in laboratory experiments, velocity is slightly increasing and temperature is almost constant up to above four inlet diameters, before the full development of the turbulence. When the turbulent regime is established, the decay of the velocity and temperature follows the trends predicted by the one-dimensional theory and observed in experiments.
The inset displays the average value of the vertical velocity and temperature fluctuations along the axis. Coherently with experimental results (George et al., 1977), velocity fluctuations reach their maximum value and a stationary trend (corresponding to about $30 \%$ of the mean value) at a lower height (about three inlet diameters) with respect to temperature fluctuations (which reach a stationary value about $40 \%$ above four inlet diameters).

\subsection{Transonic and supersonic flows}

Although not essential in the present application, the ability to solve transonic and supersonic regimes is also required for the full-scale simulation of volcanic processes. We here test the behavior of the ASHEE code in the presence of shocks in the classical Sod (1978) shock tube test case, describ- 

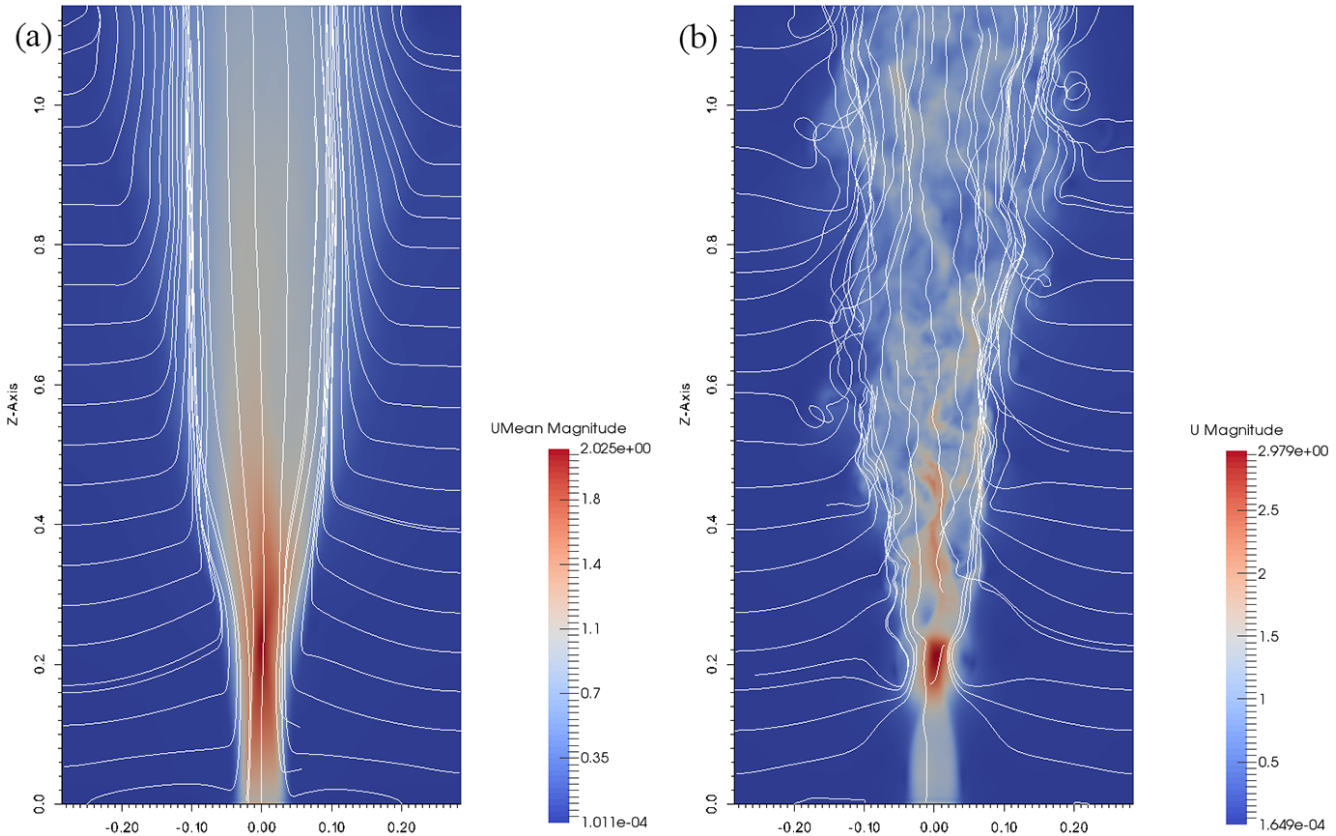

Figure 10. Two-dimensional slice and streamlines of the velocity field: (a) time-averaged velocity field; (b) instantaneous velocity field at $t=10 \mathrm{~s}$. The mean velocity field outside the plume is approximatively horizontal while in the plume it is approximately vertical. The region where the mean velocity field change direction is the region where the entrainment of air by the plume occurs.

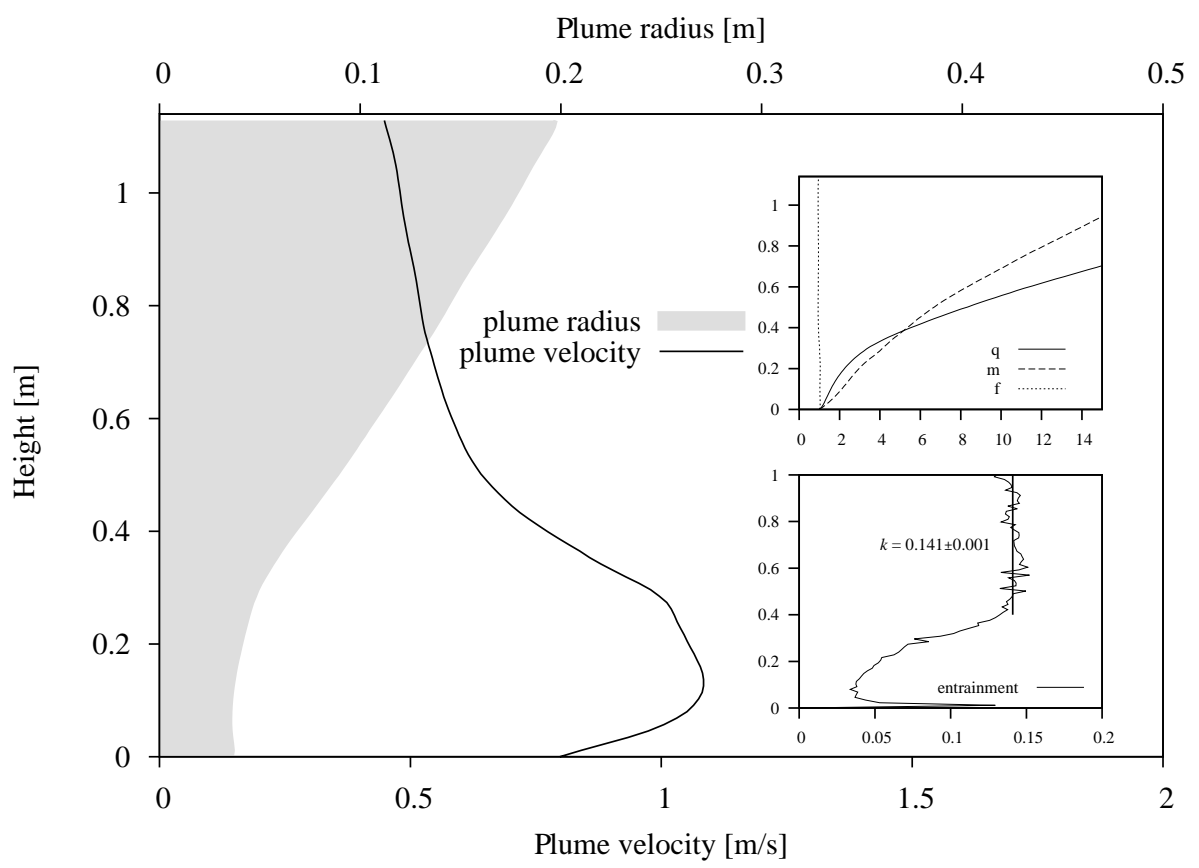

Figure 11. Time-averaged plume radius and velocity. The insets display the non-dimensional mass, momentum and buoyancy fluxes (top) and the time-averaged entrainment coefficient. The line in the entrainment panel is a constant fit, from which results $\kappa=0.141 \pm 0.001$.

ing the expansion of a compressible, single-phase gas having adiabatic index $\gamma=1.4$. At $t=0$ the domain of length $10 \mathrm{~m}$ is subdivided into two symmetric subsets. In the first subset (spatial coordinate $x<0$ ) we set $u=0, p=10^{5} \mathrm{~Pa}$,
$T=348.432 \mathrm{~K}$, so that $\rho=1 \mathrm{~kg} \mathrm{~m}^{-3}$. In the second subset $(x>0)$, we set $u=0, p=10^{4} \mathrm{~Pa}, T=278.746 \mathrm{~K}$, so that $\rho=0.125 \mathrm{~kg} \mathrm{~m}^{-3}$. We indicate with $c=374.348 \mathrm{~m} \mathrm{~s}^{-1}$ the speed of sound of the gas in the $x<0$ part of the domain. We 


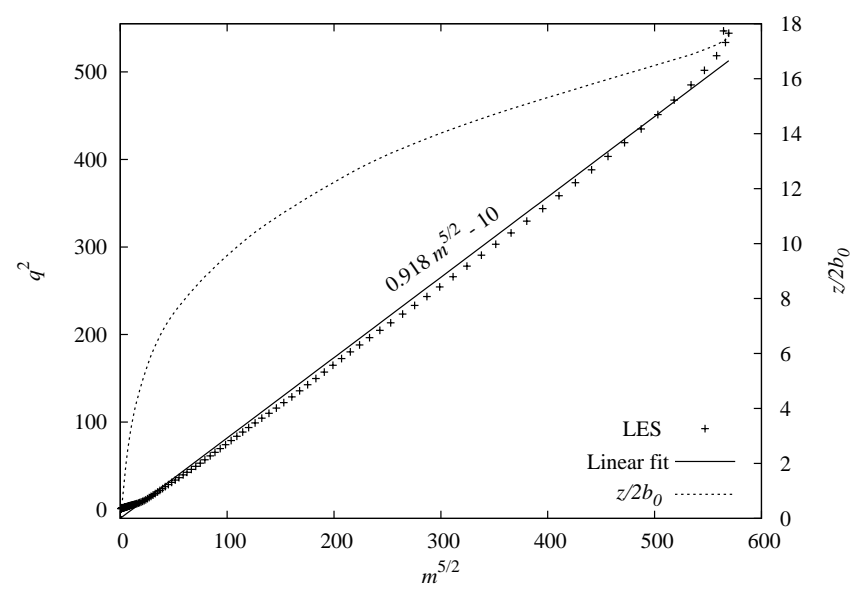

Figure 12. Linear regression between $m^{5 / 2}$ and $q^{2}$ for the plume simulation with azimuthal forcing.

impose zero gradient boundary conditions $\left(\partial_{x}(\cdot)=0\right)$ for all the variables $u, p, T$. As described in Sod (1978), a reference analytic solution exists for this problem.

In Fig. 15 we show the density profile obtained with the ASHEE model after $0.007 \mathrm{~s}$ of simulation. We performed two simulations at different resolution. The first has 100 cells and it is compared with the OpenFOAM ${ }^{\circledR}$ solver rhoCentralFoam with a second order semi-discrete, nonstaggered central scheme of Kurganov et al. (2001) for the fluxes, and a total variation diminishing limiter (Van Leer, 1997) for the interpolation. We refer to Greenshields et al. (2010) for a presentation of rhoCentralfoam and of the Sod (1978) shock tube test case. The inset of Fig. 15 is the simulation with a higher resolution (1000 cells). In this figure, the code performs satisfactorily both at low and high resolution. It is able to capture the shocks pretty well, with a diffusion that is comparable with that obtained with rhoCentralFoam, a solver conceived for simulating shocks.

\section{3-D simulation of a turbulent volcanic plume}

Numerical simulations of volcanic plumes were conducted in the framework of the IAVCEI (International Association of Volcanology and Geochemistry of the Earth Interior) plume model intercomparison initiative (Costa et al., 2015; Suzuki et al., 2016), consisting in performing a set of simulations using a standard set of input parameters so that independent results could be meaningfully compared and evaluated, and different approaches discussed. We here study threedimensional numerical simulation of a weak volcanic plume in a stratified, calm atmosphere, whose input data were set assuming parameters and meteorological conditions similar to those of the 26 January 2011 Shinmoe-dake eruption (Suzuki and Koyaguchi, 2013). Initial conditions and injection parameters are reported in Table 2.
The particle size distribution is composed of two individual classes of pyroclasts in equal weight proportion representing, respectively, fine (diameter $d=$ $0.0625 \mathrm{~mm}$; density $\hat{\rho}=2700 \mathrm{~kg} \mathrm{~m}^{-3}$, volume fraction $\epsilon=$ 0.00086821 ) and coarse ash (diameter $d=1.0000 \mathrm{~mm}$; density $\hat{\rho}=2200 \mathrm{~kg} \mathrm{~m}^{-3}$, volume fraction $\epsilon=0.00106553$ ). With respect to the laboratory benchmark case of Sect. 4.3, volcanic plumes are characterized by non-Boussinesq regimes at the vent and buoyancy reversal (with the initial mixture density about four times larger than the atmospheric one) and by a stratified atmosphere (Fig. 16). However, the most relevant difference is due to the significant temperature contrast $(900 \mathrm{~K})$ and to the presence of a high particle content which may strongly affect the mixing properties of the plume.

The Stokes number of the solid particles is, in general, a complex function of time and space, since the turbulent flow is characterized by a wide spectrum of relevant lengthand timescales. Generally, the Stokes number is associated with the most energetic turbulent eddy scale which, in analogy with laboratory plumes, has a typical turnover time of the order of $\tau_{L} \sim \operatorname{Str} \frac{2 b_{0}}{U_{0}} \approx 0.12 \mathrm{~s}$, where $b_{0}$ and $U_{0}$ are the plume radius and velocity at the vent, respectively, and Str is the Strouhal number, of the order Str $=0.3$ (Zhou et al., 2001). Based on this timescale, and computing the particle relaxation time from Eq. (3), the Stokes number for the two adopted particle classes is about $S t_{\text {coarse }} \approx 5$ and $S t_{\text {fine }} \approx 0.2$, so we expect to see non-equilibrium phenomena for both particles classes, with more evident effects on the coarsest phase. However, the average value of the Stokes number in the whole plume is not as high as calculated above. Indeed, by using Eq. (45) as reference time for the turbulent dynamics, we obtain $S t_{\text {coarse }} \approx 0.1$ and $S t_{\text {fine }} \approx 0.005$. This result has been obtained a posteriori for the finer mesh resolution, having $\xi \approx 40 \mathrm{~m}, K \approx 218 \mathrm{~m}^{2} \mathrm{~s}^{-2}$ and $\lambda_{\mathrm{T}} \approx 231 \mathrm{~m}$, when the plume reaches its maximum height. It is worth recalling here that the equilibrium-Eulerian approach is accurate and advantageous for particles having $S t \leq 0.2$ and that, in our model, we numerically limit the acceleration field in order to keep the turbulent non-equilibrium within this limit, as explained in Sect. 3 and tested in Sect. 4.2, Fig. 7. The averaged value of this limit - measuring the importance of the decoupling limiter for this simulation - is approximately $40 \%$.

The computational domain is cylindrical and extends to $483 b_{0} \times 765 b_{0}$ in the radial and vertical directions $\left(b_{0}\right.$ being the vent radius). The numerical grid is non-uniform and non-orthogonal. The discretization of the vent is represented in Fig. 17a. For the highest-resolution run, the cell size increases from a minimum grid size of $\delta=2 b_{0} / 32$ with no radial grading factor in the region where the plume is expected to develop (Fig. 17b), whose initial radius is equal to $2.5 b_{0}$. The mesh size increases linearly in the vertical direction with an angle $\theta$ such that $\tan \theta=0.147$, slightly larger than the $\tan \theta=0.096$ predicted by the Morton's plume the- 


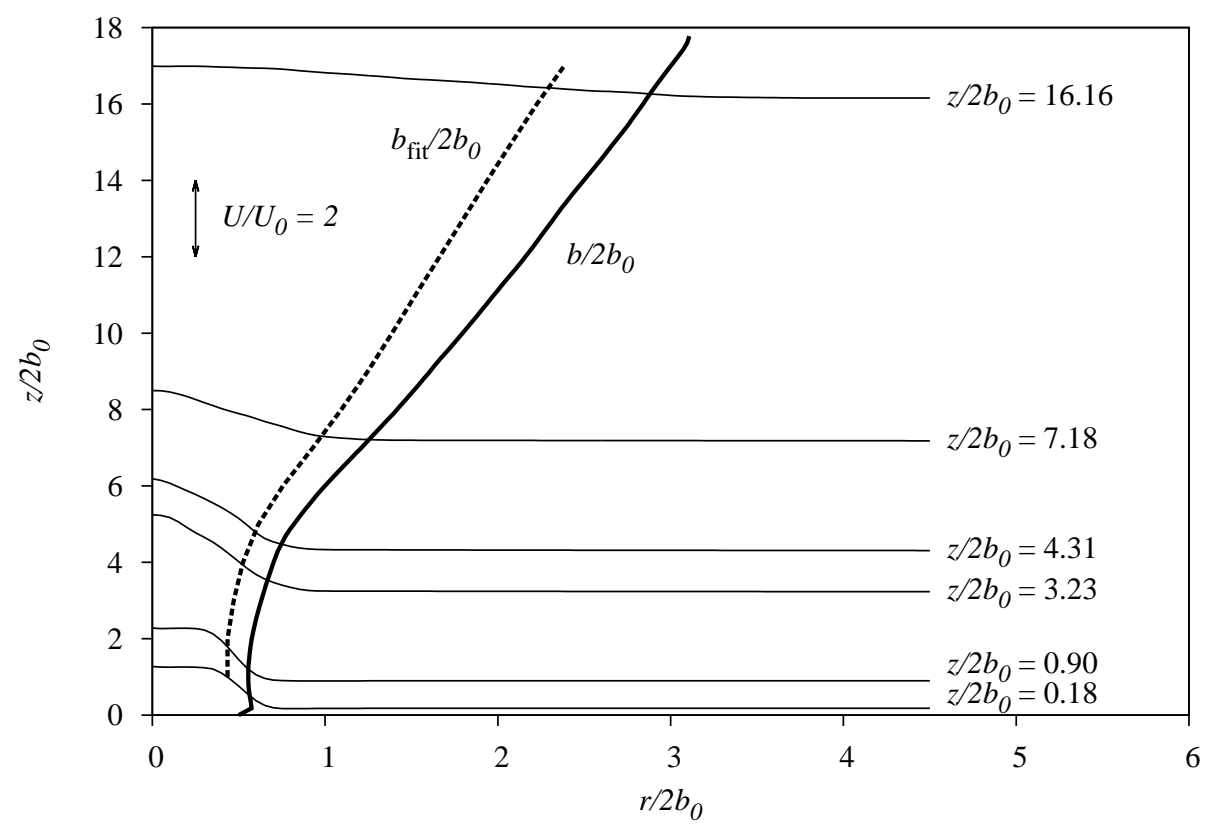

Figure 13. Radial profiles of the time-averaged velocity field at various height. The scale for these profiles is indicated by the up-down arrow on the left in the panel. The thick solid line is the plume radius evaluated from the mass, momentum and buoyancy fluxes, while the thick dashed line is the plume radius evaluated from Gaussian fits of horizontal profiles.

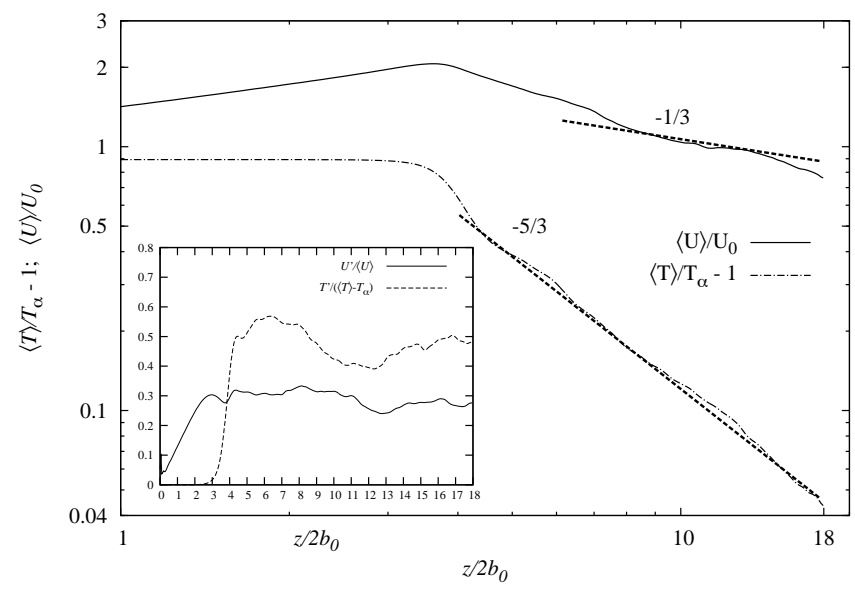

Figure 14. Centerline time-averaged axial velocity, and temperature profiles with azimuthal forcing. Inset: centerline correlations of fluctuating velocity and temperature.

ory with entrainment $\kappa=0.1$ (Ishimine, 2006). Outside this region, a radial grading factor of 1.0446 is applied. Along $z, 2048$ cells are utilized. The minimum vertical cell size is $\delta=2 b_{0} / 32$, and a grading factor of 1.00187 is imposed. The azimuthal resolution is constant and equal to $\frac{1}{32} \pi\left(5.625^{\circ}\right)$. The resulting total number of cells is $N=10747904$. This numerical mesh guarantees the accuracy of the results: the solution procedure utilizes two PISO and two PIMPLE loops to achieve an absolute residual $\epsilon_{\text {PIMPLE }}=10^{-7}$ (see Sect. 3).

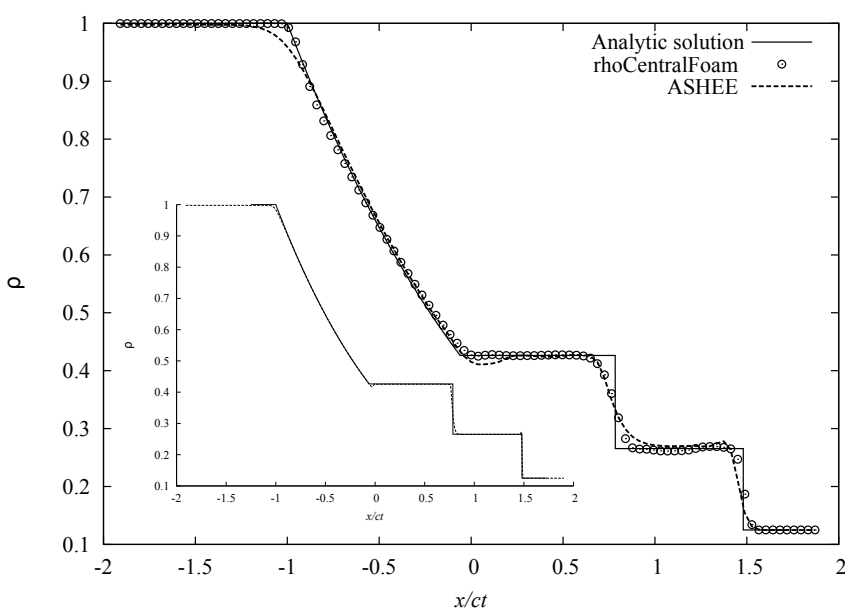

Figure 15. The Sod (1978) shock tube density after $0.007 \mathrm{~s}$ (here $c=374.348 \mathrm{~m} \mathrm{~s}^{-1}$ ). Here we compare the analytic solution (solid) with two simulations performed with ASHEE model (dashed line) and the OpenFOAM ${ }^{\circledR}$ rhocentralFoam solver (circles). The resolution is 100 cells, while in the inset the solution obtained with the ASHEE model with a resolution of 1000 cells is reported .

Simulation of $720 \mathrm{~s}$ of eruption at the highest resolution required about 490000 time steps (imposing a CFL constraint of 0.2 , resulting in an average time step $\mathrm{d} t \approx 1.5 \mathrm{~ms}$, with a maximum velocity at the vent of about $150 \mathrm{~m} \mathrm{~s}^{-1}$ ) for a total run-time of about 25 days on 1024 cores on the Fermi architecture at CINECA (meaning about $2.25 \mathrm{Mcells} \mathrm{s}^{-1}$, consistently with the estimates of Sect. 4). The lowest-resolution 

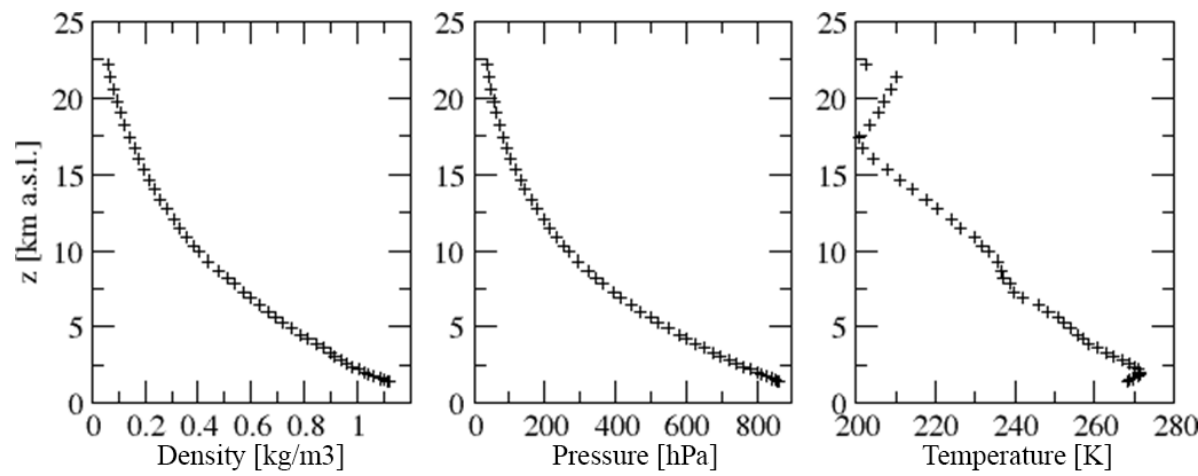

Figure 16. Atmospheric profiles as provided by the Japanese Meteorological Agency's Non-Hydrostatic Model (Hashimoto et al., 2012) for Shinmoe-dake volcano at 00:00 JST on 27 January 2011.

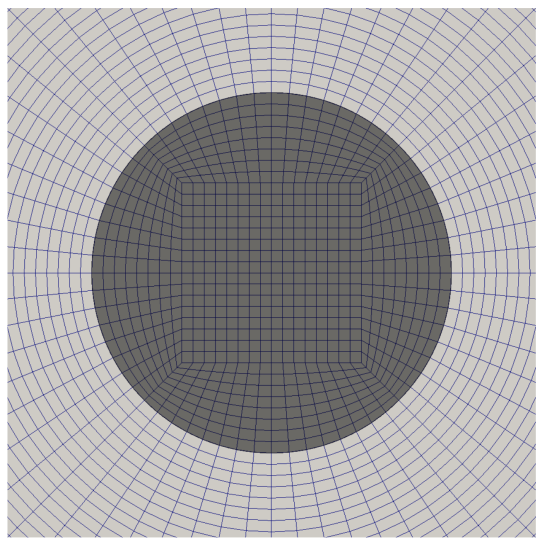

(a)

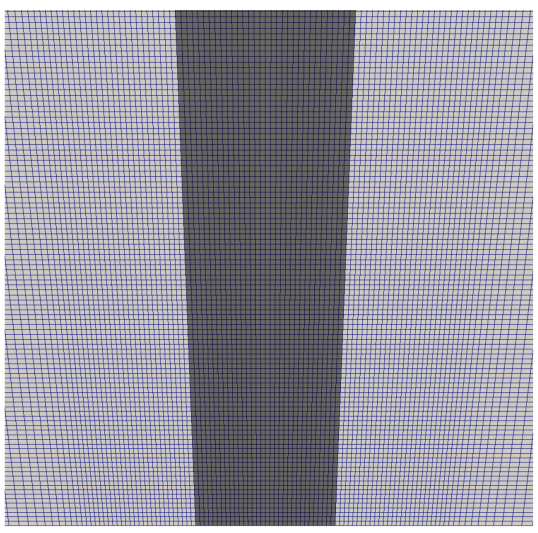

(b)

Figure 17. Close-up of the computational grid used for volcanic plume simulations.

Table 2. Vent conditions for the weak volcanic plume simulation.

\begin{tabular}{ll}
\hline Parameter & Value \\
\hline Vent elevation & $1500 \mathrm{~m}$ \\
Vent diameter & $54 \mathrm{~m}$ \\
Mass eruption rate & $1.5 \times 10^{6} \mathrm{~kg} \mathrm{~s}^{-1}$ \\
Exit velocity & $135 \mathrm{~m} \mathrm{~s}^{-1}$ \\
Exit temperature & $1273 \mathrm{~K}$ \\
Exit water fraction & $3 \mathrm{wt} \%$ \\
Mixture density at vent & $4.85 \mathrm{~kg} \mathrm{~m}^{-3}$ \\
\hline
\end{tabular}

test case ( $\left.\delta=2 b_{0} / 8\right)$, which gave satisfactory results (see Fig. 21), could be run in about $34 \mathrm{~h}$ on a quad-core Intel i7@2.8 GHz, meaning about 160000 cells/s.

Figure 18 shows the development of the volcanic plume at $t=400 \mathrm{~s}$. Because of the atmospheric stratification, the plume reaches a neutral buoyancy condition at about $10 \mathrm{~km}$ above the vent (i.e., $11.5 \mathrm{~km}$ above the sea level, still within the troposphere). Due to its inertia, the plume reaches its maximum plume height $H_{\max } \approx 12 \mathrm{~km}$, higher than the neutral buoyancy level, before spreading radially to form the so- called volcanic umbrella. The two orthogonal sections highlight the different spatial distribution of the volumetric fraction of fine (center) and coarse (right) ash particles, due to the different coupling regime with the gas phase. Coarse particles have indeed a larger settling velocity $\boldsymbol{w}_{\mathrm{s}}=\tau_{\mathrm{s}} \boldsymbol{g}$ which causes a more intense proximal fallout from the plume margins and a reduced transport by the umbrella.

Besides settling, the large inertia of the coarse ash is responsible for the kinematic decoupling, leading to preferential concentration and clustering of particles at the margins of turbulent eddies. To illustrate this phenomenon, in the nonhomogeneous flow, the instantaneous preferential concentration is computed as the (normalized) ratio between the $j$ th particle concentration and the concentration of a tracer (in our case, water vapor), i.e.,

$\mathcal{C}_{j}=\frac{y_{j}}{y_{j, 0}} \cdot \frac{y_{\text {tracer }, 0}}{y_{\text {tracer }}}$

where the 0 subscript corresponds to the value at the vent.

Figure 19a shows the distribution of $\mathcal{C}_{j}$ for the coarsest particles at $t=400 \mathrm{~s}$. The color scale is logarithmic and symmetric with respect to 1 , which corresponds to the nil prefer- 

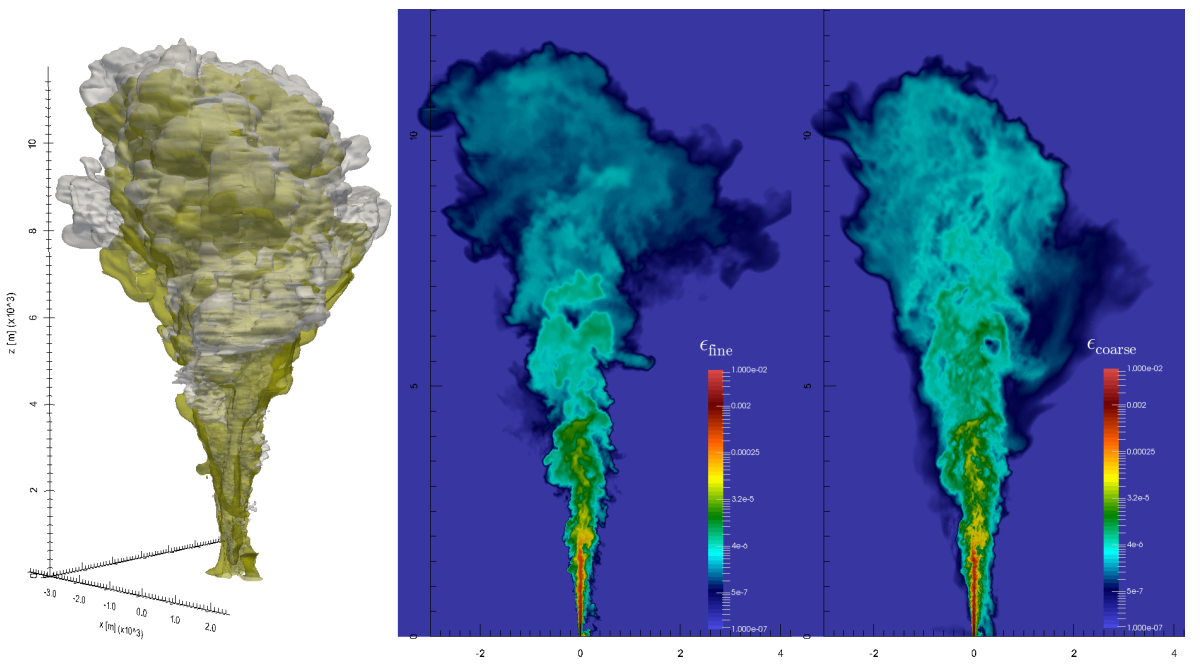

Figure 18. Three-dimensional numerical simulation of a weak volcanic plume, $400 \mathrm{~s}$ after the beginning of the injection (inlet conditions as in Table 2). The isosurfaces (left) represent fine (light gray) and coarse (light sand) ash volume fractions $\epsilon_{\mathrm{S}}=10^{-7}$. The two-dimensional plots represent the distribution of the volume concentration of fine (center) and coarse (right) particles across a vertical orthogonal slice crossing the plume axis.
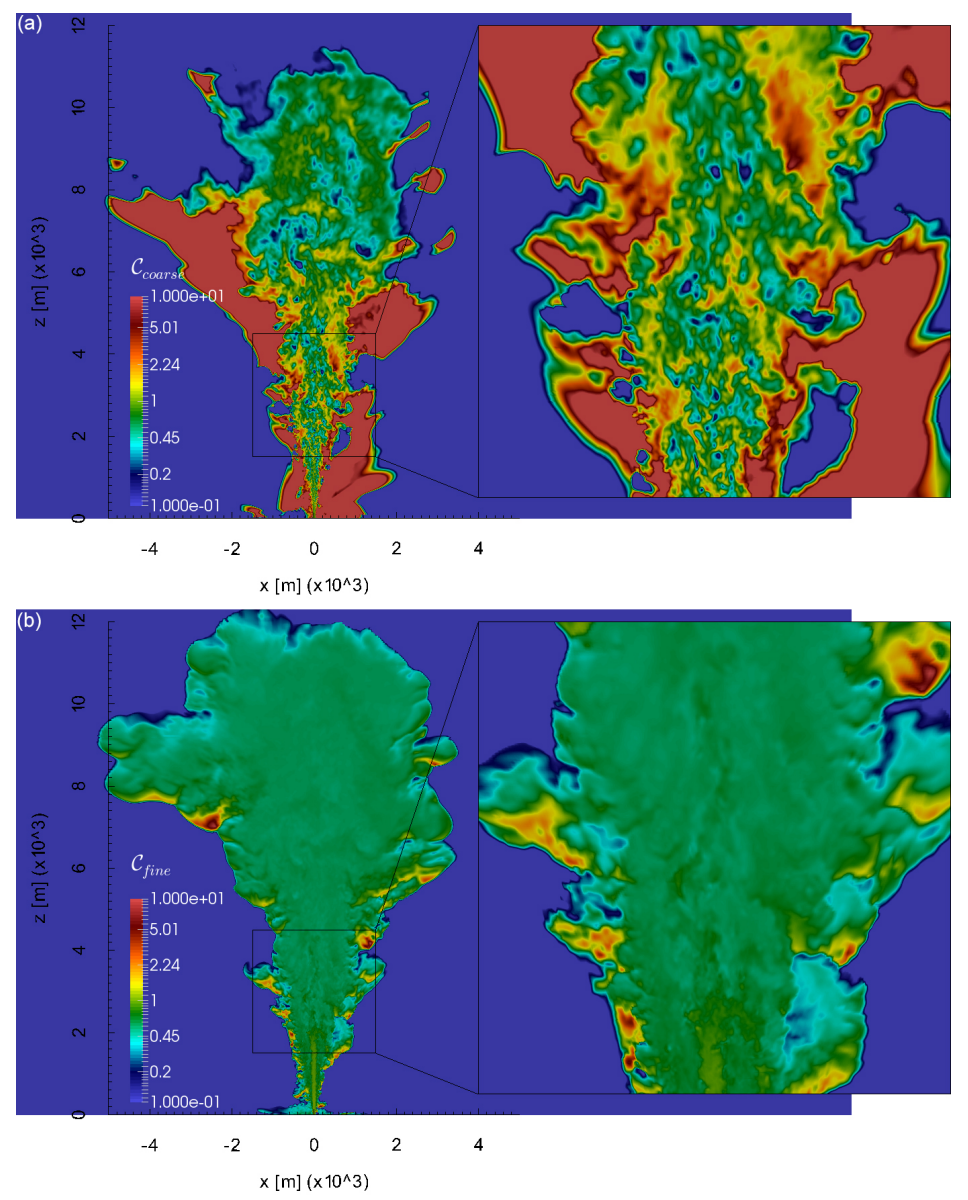

Figure 19. Distribution of $\mathcal{C}_{\text {coarse }}(\mathbf{a})$ and $\mathcal{C}_{\text {fine }}$ (b), for the coarsest particles across a vertical section at $t=400 \mathrm{~s}$ (see Eq. 52). 


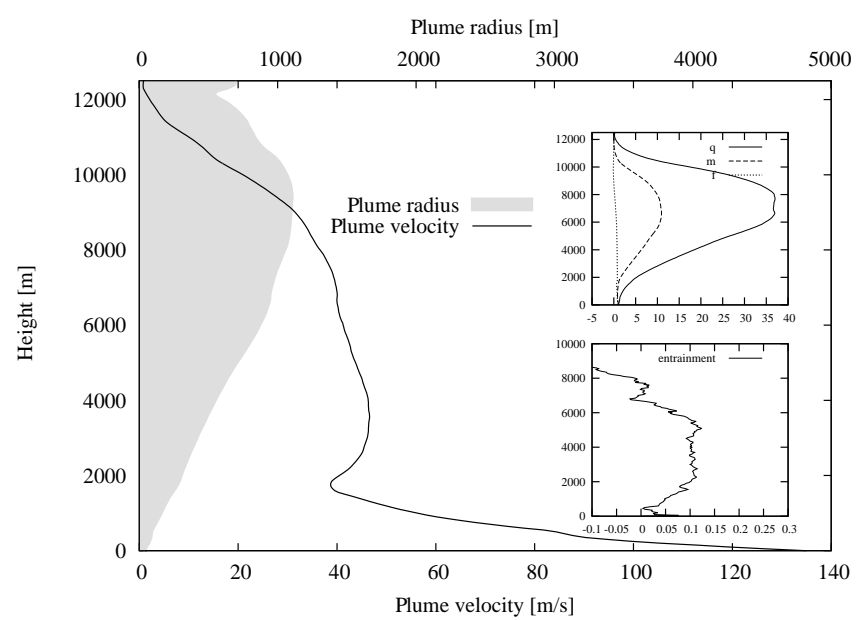

Figure 20. Time-averaged plume radius and velocity. The insets display the non-dimensional mass, momentum and buoyancy fluxes (top) and the time-averaged entrainment coefficient $\kappa$. The results refer to the simulation at maximum resolution $\delta=2 b_{0} / 32$.

ential concentration. For $\mathcal{C}_{j}<1$, the mixture is relatively depleted of particles (green to blue scale); for $\mathcal{C}_{j}>1$, particles are clustered (green to red scale), with mass fraction up to five times larger and 20 times smaller than the value it would have in absence of preferential concentration. This behavior is expected to affect the mixing and entrainment process. It is also worth remarking that the more uniform red area beyond the plume margins corresponds to the region of settling particles below the umbrella region. On the other hand, the top of the plume is relatively depleted of coarse particles. The corresponding Fig. 19b for fine particles confirms that these are tightly coupled to the gas phase and almost behave as tracers (value of $\mathcal{C}_{\text {fine }}$ is everywhere around 1). These conclusions are coherent with the a priori estimate of $S t_{j}$ we gave at the beginning of this section, based on the Taylor microscale time (Eq. 45).

Finally, we present the results obtained by averaging the volcanic plume flow field over time (in a time-window [300-720] s where the plume has reached statistically stationary conditions) and over the azimuthal angle, in order to allow comparison with one-dimensional integral models (e.g., Woods, 1988) and discuss the effect of numerical resolution. The averaging procedure is the generalization to the multiphase case of that explained in Sect. 4 (see Cerminara, 2015; Cerminara et al., 2016). Figure 20 is analogous to Fig. 11 for the laboratory plume test case and presents the results of the averaging procedure for the simulation at the highest resolution $\delta=2 b_{0} / 32$. The one-dimensional average clearly highlights the existence of a maximum plume height, where the averaged plume velocity, the mass flux $q$ and the momentum flux $m$ go to zero. In the jet stage the velocity decreases to reach a minimum of about $40 \mathrm{~m} \mathrm{~s}^{-1}$ at $z \simeq 2 \mathrm{~km}$ above the vent. Above, buoyancy reversal occurs and the plume slightly accelerates, while the radius in- creases almost linearly, reflecting the self-similar flow structure (Morton et al., 1956). The computed entrainment coefficient shows a different behavior with respect to the laboratory case, associated with the effect of the density contrast. In this case, a maximum value of about $\kappa \sim 0.1$ is obtained in the buoyant plume region between 2 and $5 \mathrm{~km}$ above the vent. Analogously to the laboratory plume case, the entertainment coefficient is much lower in the jet stage $(\kappa \simeq 0.05 \div 0.07)$. Interestingly, we find that in three-dimensional simulations the entrainment decreases near the NBL and it becomes negative above that level. This happens because the mass exits from the plume region moving into the umbrella cloud. We plan to study this behavior more thoroughly in future studies.

To analyze the effect of the grid resolution, we plot in Fig. 21 the plume radius $b(z)$ and vertical velocity $U(z)$ at resolution $\delta=2 b_{0} / 16$ (mid res.) and $\delta=2 b_{0} / 8$ (low res.). In addition, we show in the same plot the results of the dustygas model [dusty] at low res. Results demonstrate that the numerical model is robust and accurate so that even lowresolution simulations are able to capture the main features of the volcanic plume development. However, the maximum plume height systematically decreases from $12100 \mathrm{~m}$ (a), to $11300 \mathrm{~m}$ (b) to $11000 \mathrm{~m} \mathrm{(c)} \mathrm{when} \mathrm{we} \mathrm{decrease} \mathrm{the} \mathrm{resolu-}$ tion. Analogously, the Neutral Buoyancy Level (NBL) decreases from $7800 \mathrm{~m}$ (a) to $7200 \mathrm{~m}$ (b) to $7100 \mathrm{~m}$ (c). Although the lowest-resolution run seems to underestimate the maximum plume height and the plume radius by about $10 \%$, the average velocity profile is consistent in the three runs, showing a jet-plume transition at about $2000 \mathrm{~m}$ above the vent, also corresponding to the transition to a super-buoyancy region (Woods, 2010).

The dusty-gas model shows a significantly different behavior, with a larger plume radius, and a more marked jetplume transition with no further acceleration (without a super buoyancy transition). The plume height is slightly lower than the non-equilibrium case at the same resolution having maximum plume height and neutral buoyancy level of 9900 and $6100 \mathrm{~m}$, respectively. Numerical simulations thus suggest that the effects of non-equilibrium gas-particle processes (preferential concentration and settling) on air entrainment and mixing are non-negligible. These effects are certainly overlooked in the volcanological literature and will be studied more thoroughly in future studies, by applying the present model to other realistic volcanological case studies (see Cerminara et al., 2016).

\section{Conclusions}

We have developed a new, equilibrium-Eulerian model to numerically simulate compressible turbulent gas-particle flows. The model is suited to simulate relatively dilute mixtures (particle volume concentration $\epsilon_{\mathrm{s}} \lesssim 10^{-3}$ ) and particles with Stokes number $S t \lesssim 0.2$. It is appropriate to describe the dynamics of volcanic ash plumes, with kinematic decoupling 

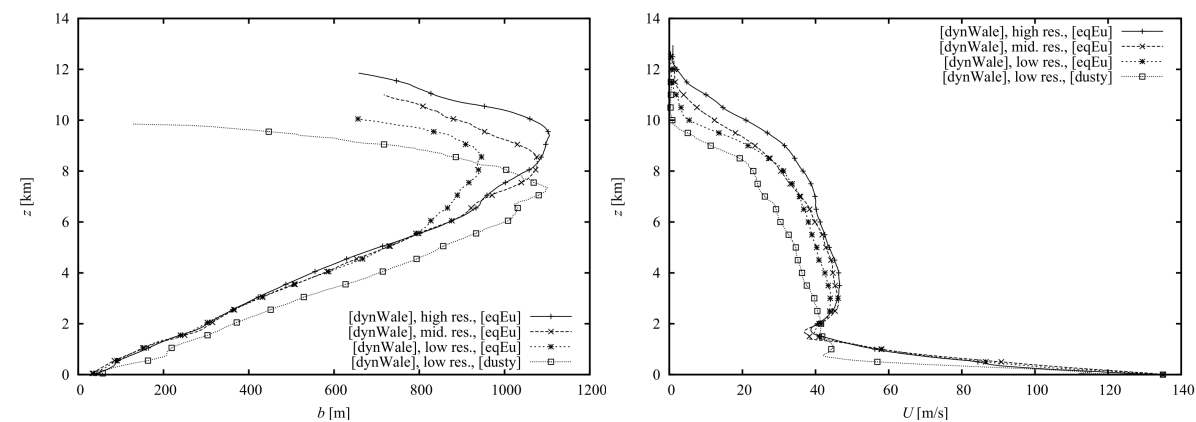

Figure 21. Time-averaged plume radius and velocity. The effect of the resolution and of the decoupling model is shown. In particular, the legend refers to the following simulations: high res. is $\delta=2 b_{0} / 32$; mid res. is $\delta=2 b_{0} / 16$; low res. is $\delta=2 b_{0} / 8$; [eqEu] refers to the equilibrium-Eulerian model; [dusty] refers to the dusty-gas model. All simulations have been performed using the same LES model.

between the gas and the particles, assumed in thermal equilibrium.

We have tested the model against controlled experiments to assess the reliability of the physical and numerical formulation and the adequacy of the model to simulate the main controlling phenomena in volcanic turbulent plumes, and in particular: (1) multiphase turbulence (including preferential concentration and density effects), (2) buoyancy and compressibility effects, (3) stratification and density nonhomogeneity.

The model reproduces the main features of volcanic plumes, namely: (1) buoyancy reversal and jet-plume transition, (2) plume maximum height and spreading of the umbrella above the neutral buoyancy level, (3) turbulent mixing and air entrainment, (4) clustering of particles, (5) proximal particle fallout. Results demonstrate that the compressible equilibrium-Eulerian approach adopted in the ASHEE model is suited to simulate the three-dimensional dynamics of volcanic plumes, being able to correctly reproduce the non-equilibrium behavior of gas-particle mixtures with a reduced computational cost with respect to that expected from Eulerian-Eulerian models.
Finally, the adopted open-source computational infrastructure, based on OpenFOAM ${ }^{\circledR}$, will make the model easily portable and usable and will ease the maintenance and implementation of new modules, making ASHEE suitable for collaborative research in different volcanological contexts.

\section{Code availability}

The ASHEE code with the input files for some simulation presented in this work are available for download on the site for collaborative volcano research and risk mitigation Vhub (https://vhub.org/groups/ashee). Code documentation is on the Volcano Modelling and Simulation gateway of INGV Pisa (http://vmsg.pi.ingv.it/). A gallery of movies of numerical simulations performed with the ASHEE code can be found at "https://sites.google.com/site/matteocerminara/". 


\section{Appendix A}

All the symbols used in this paper are listed here.

Table A1. Table of symbols.

\begin{tabular}{|c|c|}
\hline$a$ & acceleration field \\
\hline$b$ & plume radius \\
\hline $\mathbb{B}$ & subgrid-scale stress tensor \\
\hline$c$ & speed of sound \\
\hline$C$ & specific heat at constant volume \\
\hline$C_{\mathrm{D}}$ & drag coefficient \\
\hline$C_{p}$ & specific heat at constant pressure \\
\hline $\mathcal{C}_{j}$ & $\begin{array}{l}\text { normalized mass concentration } \\
\text { ratio of the } j \text { th phase }\end{array}$ \\
\hline$d$ & solid particles diameter \\
\hline $\mathcal{D}$ & $\begin{array}{l}\text { symmetrical part of the velocity } \\
\text { gradient }\end{array}$ \\
\hline $\mathcal{E}$ & turbulent energy spectrum \\
\hline$f$ & non-dimensional buoyancy flux \\
\hline$f_{j}$ & $\begin{array}{l}\text { drag force per volume unity be- } \\
\text { tween the gas and the } j \text { th solid } \\
\text { phase }\end{array}$ \\
\hline$F$ & buoyancy flux \\
\hline$g$ & gravity acceleration magnitude \\
\hline$g$ & gravity acceleration \\
\hline $\mathbb{G}$ & $\begin{array}{l}\text { equilibrium-Eulerian model op- } \\
\text { erator }\end{array}$ \\
\hline$h$ & enthalpy per unity of mass \\
\hline$H$ & plume height \\
\hline $\mathcal{H}$ & enstrophy \\
\hline$i$ & $\begin{array}{l}\text { index running over all the gas } \\
\text { phases }\end{array}$ \\
\hline$I$ & number of gas phases \\
\hline $\mathcal{I}$ & set of all the gas phases indices \\
\hline $\mathbb{I}$ & identity tensor \\
\hline$j$ & $\begin{array}{l}\text { index running over all the solid } \\
\text { phases }\end{array}$ \\
\hline$J$ & number of solid phases \\
\hline $\mathcal{J}$ & set of all the solid phases indices \\
\hline$k$ & wavenumber \\
\hline$k_{\mathrm{g}}$ & $\begin{array}{l}\text { thermal conductivity of the gas } \\
\text { phase }\end{array}$ \\
\hline$K$ & kinetic energy per unity of mass \\
\hline$L$ & length scale \\
\hline$m$ & non-dimensional momentum flux \\
\hline$M$ & momentum flux \\
\hline$N$ & number of mesh cells \\
\hline $\mathcal{O}$ & $\begin{array}{l}\text { horizontal domain, correspond- } \\
\text { ing to plume sections }\end{array}$ \\
\hline$p$ & pressure \\
\hline $\mathcal{P}$ & preferential concentration \\
\hline$q$ & non-dimensional mass flux \\
\hline$q$ & heat flux \\
\hline
\end{tabular}

Table A1. Continued.

\begin{tabular}{|c|c|}
\hline$Q$ & mass flux \\
\hline$Q_{u}$ & $\begin{array}{l}\text { second invariant of the velocity } \\
\text { gradient }\end{array}$ \\
\hline $\mathrm{Q}_{\mathrm{s}}$ & $\begin{array}{l}\text { heat exchange between the gas } \\
\text { and solid phase }\end{array}$ \\
\hline $\mathcal{Q}$ & diffusivity vector of the enthalpy \\
\hline $\mathcal{Q}_{K}$ & $\begin{array}{l}\text { diffusivity vector of the kinetic } \\
\text { energy }\end{array}$ \\
\hline$r$ & radial coordinate \\
\hline$R$ & gas constant \\
\hline$S$ & source (sink) term \\
\hline $\mathbb{S}$ & rate-of-shear tensor \\
\hline $\mathcal{S}$ & $\begin{array}{l}\text { skew-symmetric part of the ve- } \\
\text { locity gradient }\end{array}$ \\
\hline$t$ & time \\
\hline$T$ & temperature \\
\hline $\mathbb{T}$ & stress tensor \\
\hline $\mathcal{T}$ & temporal interval \\
\hline$u$ & velocity magnitude \\
\hline$u$ & velocity field \\
\hline $\boldsymbol{u}^{\prime}$ & velocity fluctuations \\
\hline$u_{\mathrm{r}}$ & $\begin{array}{l}\text { relative velocity between the gas } \\
\text { and the mixture }\end{array}$ \\
\hline$U$ & $\begin{array}{l}\text { velocity scale; plume axial veloc- } \\
\text { ity }\end{array}$ \\
\hline $\boldsymbol{v}_{j}$ & $\begin{array}{l}\text { relative velocity between the } j \text { th } \\
\text { solid and the gas phases }\end{array}$ \\
\hline $\boldsymbol{v}_{\psi}$ & $\begin{array}{l}\text { velocity correcting the mixture } \\
\text { advection term of the generic } \\
\text { field } \psi \text { due the decoupling }\end{array}$ \\
\hline$V$ & volume \\
\hline$w$ & terminal settling velocity \\
\hline$x$ & horizontal coordinate \\
\hline$x$ & position vector \\
\hline$y$ & $\begin{array}{l}\text { mass fraction; horizontal coordi- } \\
\text { nate }\end{array}$ \\
\hline $\mathcal{Y}$ & subgrid eddy diffusivity vector \\
\hline$z$ & axial coordinate \\
\hline$\alpha$ & atmospheric density \\
\hline$\beta$ & plume density \\
\hline$\gamma$ & specific heat ratio \\
\hline$\delta$ & size of the smallest mesh cell \\
\hline$\delta_{r}$ & $\begin{array}{l}\text { thickness of the turbulent bound- } \\
\text { ary layer }\end{array}$ \\
\hline$\partial_{t}$ & $\begin{array}{l}\text { partial derivative with respect to } \\
\text { time }\end{array}$ \\
\hline$\nabla$ & del operator \\
\hline$\epsilon$ & volumetric concentration \\
\hline$\epsilon$ PIMPLE & PIMPLE loop residual error \\
\hline$\varepsilon$ & turbulent dissipation \\
\hline$\eta$ & Kolmogorov length scale \\
\hline$\kappa$ & entrainment coefficient \\
\hline$\varkappa_{i}$ & $\begin{array}{l}\text { molar fraction of the } i \text { th gas com- } \\
\text { ponent }\end{array}$ \\
\hline
\end{tabular}


Table A1. Continued.

\begin{tabular}{|c|c|}
\hline$\lambda_{\mathrm{p}-\mathrm{p}}$ & mean free path of the particles \\
\hline$\lambda_{\mathrm{T}}$ & Taylor microscale \\
\hline$\mu$ & dynamic viscosity \\
\hline$v$ & kinematic viscosity \\
\hline$\xi$ & LES length scale \\
\hline$\hat{\rho}$ & density \\
\hline$\rho$ & bulk density \\
\hline$\tau$ & typical timescale \\
\hline$\tau_{j}$ & Stokes time of the $j$ th solid phase \\
\hline$\tau_{T}$ & $\begin{array}{l}\text { Stokes time of the thermal decou- } \\
\text { pling }\end{array}$ \\
\hline$\phi_{\mathrm{c}}$ & $\begin{array}{l}\text { correction factor to the Stokes } \\
\text { time }\end{array}$ \\
\hline$\psi$ & generic flow field \\
\hline$\Omega$ & spatial domain \\
\hline Co & Courant number \\
\hline$M a$ & Mach number \\
\hline $\mathrm{Nu}$ & Nusselt number \\
\hline $\mathrm{Pr}$ & Prandtl number \\
\hline $\operatorname{Re}$ & Reynolds number \\
\hline St & Stokes number \\
\hline Str & Strouhal number \\
\hline$(\cdot)_{0}$ & $\begin{array}{l}\text { relative to the initial time; relative } \\
\text { to the vent level }\end{array}$ \\
\hline & compressible part \\
\hline$(\cdot)_{\text {coarse }}$ & relative to the coarse particles \\
\hline & relative to the eddy turnover scale \\
\hline$(\cdot)_{\text {fine }}$ & relative to the fine particles \\
\hline$(\cdot)_{\text {fit }}$ & relative to the Gaussian fit \\
\hline$(\cdot) \mathrm{g}$ & relative to the gas phase \\
\hline$(\cdot)_{i}$ & relative to the $i$ th solid phase \\
\hline$(\cdot)_{j}$ & relative to the $j$ th solid phase \\
\hline$(\cdot)_{k}$ & relative to the wavenumber $k$ \\
\hline$(\cdot)_{L}$ & relative to the length scale $L$ \\
\hline$(\cdot)_{\mathrm{m}}$ & relative to the mixture \\
\hline$(\cdot)_{\max }$ & maximum value \\
\hline$(\cdot)_{\min }$ & minimum value \\
\hline$(\cdot)_{\mathrm{rms}}$ & root-mean-square \\
\hline$(\cdot)_{\mathrm{s}}$ & relative to the solid phase \\
\hline$(\cdot)_{\text {tracer }}$ & relative to the tracer \\
\hline$(\cdot)_{z}$ & axial component \\
\hline$(\cdot)_{\alpha}$ & relative to the atmosphere \\
\hline$(\cdot)_{\eta}$ & $\begin{array}{l}\text { relative to the Kolmogorov scale } \\
\eta\end{array}$ \\
\hline $\begin{array}{l}(\cdot)_{\lambda} \\
(\cdot)_{\xi}\end{array}$ & $\begin{array}{l}\text { relative to the Taylor microscale } \\
\text { relative to the LES scale } \xi\end{array}$ \\
\hline$\overline{(\cdot)}$ & $\begin{array}{l}\text { filtered quantity; time averaged } \\
\text { quantity }\end{array}$ \\
\hline$\tilde{(\cdot)}$ & Favre-filtered quantity \\
\hline$\langle\cdot\rangle_{j}$ & $\begin{array}{l}\text { spatial averaged quantity, with } \\
\text { weight } y_{i}\end{array}$ \\
\hline$\langle\cdot\rangle_{\Omega}$ & $\begin{array}{l}\text { quantity averaged over the do- } \\
\text { main } \Omega\end{array}$ \\
\hline
\end{tabular}




\section{Appendix B: Derivation of the Eulerian-Eulerian model in "mixture" formulation}

In the regime described in Sect. 2, the Eulerian-Eulerian equations for a mixture of a gas and a solid dispersed phase are (Marble, 1970; Gidaspow, 1994; Neri et al., 2003; Feireisl, 2004; Esposti Ongaro et al., 2008; Garnier et al., 2009; Berselli et al., 2015)

$\partial_{t} \rho_{i}+\nabla \cdot\left(\rho_{i} \boldsymbol{u}_{\mathrm{g}}\right)=0, \quad i \in \mathcal{I} ;$

$\partial_{t} \rho_{j}+\nabla \cdot\left(\rho_{j} \boldsymbol{u}_{j}\right)=S_{j}, \quad j \in \mathcal{J} ;$

$\partial_{t}\left(\rho_{\mathrm{g}} \boldsymbol{u}_{\mathrm{g}}\right)+\nabla \cdot\left(\rho_{\mathrm{g}} \boldsymbol{u}_{\mathrm{g}} \otimes \boldsymbol{u}_{\mathrm{g}}\right)+\nabla p$

$=\nabla \cdot \mathbb{T}+\rho_{\mathrm{g}} \boldsymbol{g}-\sum_{j \in \mathcal{J}} \boldsymbol{f}_{j} ;$

$\partial_{t}\left(\rho_{j} \boldsymbol{u}_{j}\right)+\nabla \cdot\left(\rho_{j} \boldsymbol{u}_{j} \otimes \boldsymbol{u}_{j}\right)=\rho_{j} \boldsymbol{g}$

$+\boldsymbol{f}_{j}+S_{j} \boldsymbol{u}_{j}, \quad j \in \mathcal{J}$;

$\partial_{t}\left(\rho_{\mathrm{g}} h_{\mathrm{g}}\right)+\nabla \cdot\left(\rho_{\mathrm{g}} h_{\mathrm{g}} \boldsymbol{u}_{\mathrm{g}}\right)+\nabla \cdot\left(\boldsymbol{q}-\mathbb{T} \cdot \boldsymbol{u}_{\mathrm{g}}\right)$

$=\partial_{t} p-\partial_{t}\left(\rho_{\mathrm{g}} K_{\mathrm{g}}\right)-\nabla \cdot\left(\rho_{\mathrm{g}} K_{\mathrm{g}} \boldsymbol{u}_{\mathrm{g}}\right)+\rho_{\mathrm{g}}\left(\boldsymbol{g} \cdot \boldsymbol{u}_{\mathrm{g}}\right)$

$-\sum_{j \in \mathcal{J}}\left(\boldsymbol{u}_{j} \cdot \boldsymbol{f}_{j}+\mathrm{Q}_{j}\right)$;

$\partial_{t}\left(\rho_{j} h_{j}\right)+\nabla \cdot\left(\rho_{j} h_{j} \boldsymbol{u}_{j}\right)=\mathrm{Q}_{j}+S_{j} h_{j}, \quad j \in \mathcal{J} ;$

which are the $I+4+5 J$ balance laws of mass, momentum and enthalpy of the gaseous and solid phases, respectively.

In this formulation the equations are solved for each phase singularly. However, when the Stokes number is small, and consequently the coupling between the phases is strong, the numerical solution of this formulation can be demanding, because the coupling terms $\boldsymbol{f}_{j}$ (Eq. 2) and $\mathrm{Q}_{j}$ (Eq. 7) become very important. An alternative is to reformulate the whole problem in terms of the mixture fields $\left(\rho_{\mathrm{m}}, \boldsymbol{u}_{\mathrm{m}}, h_{\mathrm{m}}\right)$, using the explicit form of the coupling terms only to determine the velocity and temperature difference between the phases.

We show in what follows how Eq. (B1) can be expressed in terms of the mixture fields.

\section{B1 Advection in mixture formulation}

Let the particle velocity field be $\boldsymbol{u}_{j}=\boldsymbol{u}_{\mathrm{g}}+\boldsymbol{v}_{j}$. Recalling the definition for the mass fraction and the mixture density given at the beginning of Sect. 2.1, we here define the mixture velocity field $\boldsymbol{u}_{\mathrm{m}}$ and the relative velocity between the gas and the mixture $\boldsymbol{u}_{\mathrm{r}}$ through the mass weighted average:

$$
\boldsymbol{u}_{\mathrm{m}}=\sum_{i \in \mathcal{I}} y_{i} \boldsymbol{u}_{\mathrm{g}}+\sum_{j \in \mathcal{J}} y_{j} \boldsymbol{u}_{j}
$$

$\boldsymbol{u}_{\mathrm{g}}=\boldsymbol{u}_{\mathrm{m}}+\boldsymbol{u}_{\mathrm{r}}$ $\boldsymbol{u}_{\mathrm{r}}=-\sum_{j \in \mathcal{J}} y_{j} \boldsymbol{v}_{j}$

Using these definitions, the advection of a generic field $\psi$ can be rewritten; $\sum_{\mathcal{I}} \rho_{i} \psi_{i}+\sum_{\mathcal{J}} \rho_{j} \psi_{j}=\rho_{\mathrm{m}} \psi_{\mathrm{m}}$;

$$
\begin{aligned}
& \sum_{i \in \mathcal{I}} \rho_{i} \psi_{i} \boldsymbol{u}_{\mathrm{g}}+\sum_{j \in \mathcal{J}} \rho_{j} \psi_{j} \boldsymbol{u}_{j}=\rho_{\mathrm{m}} \psi_{\mathrm{m}} \boldsymbol{u}_{\mathrm{m}} \\
& +\rho_{\mathrm{m}} \sum_{j \in \mathcal{J}} y_{j} \boldsymbol{v}_{j}\left(\psi_{j}-\psi_{\mathrm{m}}\right)=\rho_{\mathrm{m}} \psi_{\mathrm{m}}\left(\boldsymbol{u}_{\mathrm{m}}+\boldsymbol{v}_{\psi}\right),
\end{aligned}
$$

where

$\boldsymbol{v}_{\psi}=\frac{\sum_{\mathcal{J}} y_{j} \boldsymbol{v}_{j}\left(\psi_{j}-\psi_{\mathrm{m}}\right)}{\psi_{\mathrm{m}}}$

can be defined where $\psi_{\mathrm{m}} \neq 0$. This velocity field takes into account the kinematic decoupling $\boldsymbol{v}_{j}$, correcting the advection term of $\psi_{\mathrm{m}}$.

\section{B2 Continuity equations}

Summing up over $i$ and $j$ in Eqs. (B1a) and (B1b), we obtain the continuity equation for the mixture:

$\partial_{t} \rho_{\mathrm{m}}+\nabla \cdot\left(\rho_{\mathrm{m}} \boldsymbol{u}_{\mathrm{m}}\right)=\sum_{j \in \mathcal{J}} S_{j}$,

while for the phases we have

$$
\begin{aligned}
\partial_{t}\left(\rho_{\mathrm{m}} y_{i}\right)+\nabla \cdot\left(\rho_{\mathrm{m}} \boldsymbol{u}_{\mathrm{g}} y_{i}\right) & =0, \quad i \in \mathcal{I} ; \\
\partial_{t}\left(\rho_{\mathrm{m}} y_{j}\right)+\nabla \cdot\left(\rho_{\mathrm{m}} \boldsymbol{u}_{j} y_{j}\right) & =S_{j}, \quad j \in \mathcal{J} .
\end{aligned}
$$

It is worth noting that the mixture density follows the classical continuity equation with velocity field $\boldsymbol{u}_{\mathrm{m}}$.

\section{B3 Momentum equation}

Summing up over $i$ and $j$ the gas and particle momentum Eqs. (B1c) and (B1d), and using Eq. (B5) with $\psi=\boldsymbol{u}$, we obtain

$$
\begin{aligned}
\partial_{t}\left(\rho_{\mathrm{m}} \boldsymbol{u}_{\mathrm{m}}\right) & +\nabla \cdot\left(\rho_{\mathrm{m}} \boldsymbol{u}_{\mathrm{m}} \otimes \boldsymbol{u}_{\mathrm{m}}+\rho_{\mathrm{m}} \mathbb{T}_{\mathrm{r}}\right)+\nabla p \\
& =\nabla \cdot \mathbb{T}+\rho_{\mathrm{m}} \boldsymbol{g}+\sum_{j \in \mathcal{J}} S_{j} \boldsymbol{u}_{j},
\end{aligned}
$$

where $\mathbb{T}_{\mathrm{r}}=\sum_{\mathcal{J}}\left(y_{j} \boldsymbol{v}_{j} \otimes \boldsymbol{v}_{j}\right) \boldsymbol{u}_{\mathrm{r}} \otimes \boldsymbol{u}_{\mathrm{r}}$. This equation is the classical compressible Navier-Stokes equation with the substitution $\boldsymbol{u}_{\mathrm{g}} \rightarrow \boldsymbol{u}_{\mathrm{m}}$ and the addition of the term $\nabla \cdot\left(\rho_{\mathrm{m}} \mathbb{T}_{\mathrm{r}}\right)$ which takes into account the effects of particle decoupling on momentum (two-way coupling). 


\section{B4 Enthalpy equation}

The same technique can be used for the enthalpy in Eq. (B1). By defining

$$
\begin{aligned}
h_{\mathrm{m}} & =\sum_{\mathcal{I}} y_{i} h_{i}+\sum_{\mathcal{J}} y_{j} h_{j} \\
K_{\mathrm{m}} & =\sum_{\mathcal{I}} y_{i} K_{i}+\sum_{\mathcal{J}} y_{j} K_{j}=\frac{1}{2}\left|\boldsymbol{u}_{\mathrm{m}}\right|^{2} \\
& +\frac{1}{2} \sum_{\mathcal{J}} y_{j}\left|\boldsymbol{v}_{j}\right|^{2}-\frac{1}{2}\left|\boldsymbol{u}_{\mathrm{r}}\right|^{2},
\end{aligned}
$$

summing up all the enthalpy Eqs. (B1f) and (B1e), and using Eq. (B5) with $\psi=h$ and $\psi=K$, we obtain

$$
\begin{aligned}
& \partial_{t}\left(\rho_{\mathrm{m}} h_{\mathrm{m}}\right)+\nabla \cdot\left[\rho_{\mathrm{m}} h_{\mathrm{m}}\left(\boldsymbol{u}_{\mathrm{m}}+\boldsymbol{v}_{h}\right)\right]-\nabla \cdot\left(\mathbb{T} \cdot \boldsymbol{u}_{\mathrm{g}}-\boldsymbol{q}\right)= \\
& =\partial_{t} p-\partial_{t}\left(\rho_{\mathrm{m}} K_{\mathrm{m}}\right)-\nabla \cdot\left[\rho_{\mathrm{m}} K_{\mathrm{m}}\left(\boldsymbol{u}_{\mathrm{m}}+\boldsymbol{v}_{K}\right)\right]+\rho_{\mathrm{m}}\left(\boldsymbol{g} \cdot \boldsymbol{u}_{\mathrm{m}}\right) \\
& +\sum_{j \in \mathcal{J}} S_{j}\left(h_{j}+K_{j}\right),
\end{aligned}
$$

where

$$
\begin{aligned}
& \boldsymbol{v}_{h}=\boldsymbol{u}_{\mathrm{r}}+\frac{\sum_{\mathcal{J}} y_{j} h_{j} \boldsymbol{v}_{j}}{h_{\mathrm{m}}}=\frac{\sum_{\mathcal{J}} y_{j}\left(h_{j}-h_{\mathrm{m}}\right) \boldsymbol{v}_{j}}{h_{\mathrm{m}}} \\
& \boldsymbol{v}_{K}=\boldsymbol{u}_{\mathrm{r}}+\frac{\sum_{\mathcal{J}} y_{j} K_{j} \boldsymbol{v}_{j}}{K_{\mathrm{m}}}=\frac{\sum_{\mathcal{J}} y_{j}\left(K_{j}-K_{\mathrm{m}}\right) \boldsymbol{v}_{j}}{K_{\mathrm{m}}}
\end{aligned}
$$

are the correction velocity fields taking into account the combined effect due to kinematic decoupling and difference between the enthalpy $\left(\boldsymbol{v}_{h}\right)$ and kinetic energy $\left(\boldsymbol{v}_{K}\right)$ of the mixture and of the $j$ th species.

\section{B5 Decoupling}

The great advantage of Eqs. (B10) and (B13) is that the coupling terms $\boldsymbol{f}_{j}$ and $\mathrm{Q}_{j}$ cancel out when summing the equations. The equations for the mixture momentum and enthalpy differ from the corresponding single-phase equations only because of the new decoupling terms (those proportional to $\mathbb{T}_{\mathrm{r}}, \boldsymbol{v}_{h}, \boldsymbol{v}_{K}$ ), which are small in the strongly coupled regime (they goes to zero in the dusty-gas and in the one-way coupling approximations). All the $I+4+J$ equations of mass, momentum and enthalpy conservation for the mixture are summarized in Eq. (10).

However, we started with $I+4+5 J$ equations. The remaining $4 J$ equations are those modeling the decoupling, which depend on $f_{j}$ and $\mathrm{Q}_{j}$. They are the $3 J$ momentum and $J$ enthalpy equations for the solid phases. They should be solved together with the mixture equations in order to find the kinematic decoupling $\boldsymbol{v}_{j}$ and the thermal decoupling $T_{j}-T_{\mathrm{g}}$. Another possibility is to use the equilibrium-Eulerian model described in Sect. 2.2.

\section{Appendix C: Derivation of the equilibrium-Eulerian approximation through asymptotic expansion}

Equation (13) is nonlinear because of the convective term $\boldsymbol{u}_{j}$. $\nabla \boldsymbol{u}_{j}$ but also because of the correction term $\phi_{\mathrm{c}}\left(R e_{j}\right)\left(\boldsymbol{u}_{\mathrm{g}}-\boldsymbol{u}_{j}\right)$ in the Stokes drag force (see Eq. 2). As pointed out and analyzed in Wang and Maxey (1993), the latter nonlinear term can be considered as slowly variable and treated as a constant in the following analysis.

Here we want to solve Eq. (13) using an asymptotic expansion technique. Indeed, letting $1 / \tau_{j} \rightarrow+\infty$ and considering $t \gg \tau_{j}$, it is possible to formally solve that equation. In our volcanological applications there are some zones in the domain where the gravitational effect (particle fallout) is dominant, thus we must consider the term $\boldsymbol{w}_{j}=\tau_{j} \boldsymbol{g}$ at the leading order. In other words, we must consider $\boldsymbol{g}=O\left(\boldsymbol{u}_{\mathrm{g}} / \tau_{j}\right)$ and rewrite Eq. (13) in terms of the terminal velocity $\boldsymbol{w}_{j}=\tau_{j} \boldsymbol{g}$ already defined in Eq. (5). Then, multiplying Eq. (13) by $e^{t / \tau_{j}}$ and calling $\boldsymbol{V}=\boldsymbol{u}_{j} e^{t / \tau_{j}}$, we get

$\partial_{t} \boldsymbol{V}+\boldsymbol{u}_{j} \cdot \nabla \boldsymbol{V}=\left(\frac{1}{\tau_{j}}\left(\boldsymbol{u}_{\mathrm{g}}+\boldsymbol{w}_{j}\right)\right) e^{t / \tau_{j}}$,

which is a transport equation, with solution

$\boldsymbol{V}\left(\boldsymbol{X}\left(\boldsymbol{x}_{0}, t\right), t\right)=\boldsymbol{V}_{0}\left(\boldsymbol{x}_{0}\right)$

$+\int_{0}^{t} \frac{1}{\tau_{j}}\left(\boldsymbol{u}_{\mathrm{g}}\left(\boldsymbol{X}\left(\boldsymbol{x}_{0}, s\right), s\right)+\boldsymbol{w}_{j}\right) e^{s / \tau_{j}} \mathrm{~d} s$,

with $\boldsymbol{X}\left(\boldsymbol{x}_{0}, t\right)$ such that

$\mathrm{d}_{t} \boldsymbol{X}\left(\boldsymbol{x}_{0}, t\right)=\boldsymbol{u}_{j}\left(\boldsymbol{X}\left(\boldsymbol{x}_{0}, t\right), t\right)$

$X\left(x_{0}, 0\right)=x_{0}$.

Thus we have formally obtained $\boldsymbol{u}_{j}$ :

$\boldsymbol{u}_{j}\left(\boldsymbol{X}\left(\boldsymbol{x}_{0}, t\right), t\right)=\boldsymbol{u}_{j, 0}\left(\boldsymbol{x}_{0}\right) e^{-t / \tau_{j}}+\left(1-e^{-t / \tau_{j}}\right) \boldsymbol{w}_{j}$

$+\int_{0}^{t} \frac{1}{\tau_{j}} \boldsymbol{u}_{\mathrm{g}}\left(\boldsymbol{X}\left(\boldsymbol{x}_{0}, t-s\right), t-s\right) e^{-\frac{s}{\tau_{j}}} \mathrm{~d} s$,

where $\boldsymbol{u}_{j}\left(\boldsymbol{X}\left(\boldsymbol{x}_{0}, t\right), t\right)$ is the velocity of the particle " $\boldsymbol{x}_{0}$ " evaluated in its position at time $t$. In order to carry out the asymptotic expansion, we perform the Taylor expansion of $\boldsymbol{u}_{\mathrm{g}}$ around $s=0$ :

$\boldsymbol{u}_{\mathrm{g}}\left(\boldsymbol{X}\left(\boldsymbol{x}_{0}, t-s\right), t-s\right)=\sum_{n=0}^{+\infty} \frac{(-1)^{n}}{n !} \frac{\mathrm{d}^{n} \boldsymbol{u}_{\mathrm{g}}}{\mathrm{d} t^{n}}\left(\boldsymbol{X}\left(\boldsymbol{x}_{0}, t\right), t\right) s^{n}$.

Using the relation

$\int \frac{1}{\tau_{j}} s^{n} e^{-\frac{s}{\tau_{j}}} \mathrm{~d} s=-e^{-\frac{s}{\tau_{j}}} \sum_{k=0}^{n} \frac{n !}{k !} \tau_{j}^{n-k} s^{k}$

and supposing that the series converges uniformly, we get 


$$
\begin{aligned}
& \int_{0}^{t} \frac{1}{\tau_{j}} \boldsymbol{u}_{\mathrm{g}}\left(\boldsymbol{X}\left(\boldsymbol{x}_{0}, t-s\right), t-s\right) e^{-\frac{s}{\tau_{j}}} \mathrm{~d} s \\
& =\int_{0}^{t} \frac{1}{\tau_{j}} \sum_{n=0}^{+\infty} \frac{(-1)^{n}}{n !} \frac{\mathrm{d}^{n} \boldsymbol{u}_{\mathrm{g}}}{\mathrm{d} t^{n}}\left(\boldsymbol{X}\left(\boldsymbol{x}_{0}, t\right), t\right) s^{n} e^{-\frac{s}{\tau_{j}}} \mathrm{~d} s \\
& =-\left.\sum_{n=0}^{+\infty}(-1)^{n} \frac{\mathrm{d}^{n} \boldsymbol{u}_{\mathrm{g}}}{\mathrm{d} t^{n}}\left(\boldsymbol{X}\left(\boldsymbol{x}_{0}, t\right), t\right) e^{-\frac{s}{\tau_{j}}} \sum_{k=0}^{n} \frac{1}{k !} \tau_{j}^{n-k} s^{k}\right|_{0} ^{t} \\
& =\sum_{n=0}^{+\infty}(-1)^{n} \tau_{j}^{n} \frac{\mathrm{d}^{n} \boldsymbol{u}_{\mathrm{g}}}{\mathrm{d} t^{n}}\left[1-\sum_{k=0}^{n} \frac{1}{k !}\left(\frac{t}{\tau_{j}}\right)^{k} e^{-\frac{t}{\tau_{j}}}\right] .
\end{aligned}
$$

Thus

$$
\begin{aligned}
& \boldsymbol{u}_{j}\left(\boldsymbol{X}\left(\boldsymbol{x}_{0}, t\right), t\right)-\boldsymbol{u}_{\mathrm{g}}\left(\boldsymbol{X}\left(\boldsymbol{x}_{0}, t\right), t\right)-\boldsymbol{w}_{j}=\left(\boldsymbol{u}_{j, 0}\left(\boldsymbol{x}_{0}\right)\right. \\
& \left.-\boldsymbol{u}_{\mathrm{g}}\left(\boldsymbol{X}\left(\boldsymbol{x}_{0}, t\right), t\right)-\boldsymbol{w}_{j}\right) e^{-t / \tau_{j}} \\
& -\tau_{j} \frac{\mathrm{d} \boldsymbol{u}_{\mathrm{g}}}{\mathrm{d} t}\left(\boldsymbol{X}\left(\boldsymbol{x}_{0}, t\right), t\right)\left(1-e^{-t / \tau_{j}}-\frac{t}{\tau_{j}} e^{-t / \tau_{j}}\right) \\
& +\tau_{j}^{2} \frac{\mathrm{d}^{2} \boldsymbol{u}_{\mathrm{g}}}{\mathrm{d} t^{2}}\left(1-e^{-t / \tau_{j}}-\frac{t}{\tau_{j}} e^{-t / \tau_{j}}-\frac{1}{2}\left(\frac{t}{\tau_{j}}\right)^{2} e^{-t / \tau_{j}}\right) \\
& +O\left(\tau_{j}^{3}\right) .
\end{aligned}
$$

If now we consider $t \gg \tau_{j}$, neglecting the transient phase in which particles reach the equilibrium with the fluid ${ }^{1}$, we obtain

$$
\begin{aligned}
& \boldsymbol{u}_{j}(\boldsymbol{X}(\boldsymbol{x}, t), t)=\boldsymbol{u}_{\mathrm{g}}(\boldsymbol{X}(\boldsymbol{x}, t), t)+\boldsymbol{w}_{j}-\tau_{j} \frac{\mathrm{d} \boldsymbol{u}_{\mathrm{g}}}{\mathrm{d} t}(\boldsymbol{X}(\boldsymbol{x}, t), t) \\
& +\tau_{j}^{2} \frac{\mathrm{d}^{2} \boldsymbol{u}_{\mathrm{g}}}{\mathrm{d} t^{2}}(\boldsymbol{X}(\boldsymbol{x}, t), t)+O\left(\tau_{j}^{3}\right),
\end{aligned}
$$

which, using Eq. (C3), gives us

$\boldsymbol{u}_{j}=\boldsymbol{u}_{\mathrm{g}}+\boldsymbol{w}_{j}-\tau_{j}\left(\partial_{t} \boldsymbol{u}_{\mathrm{g}}+\boldsymbol{u}_{j} \cdot \nabla \boldsymbol{u}_{\mathrm{g}}\right)+O\left(\tau_{j}^{2}\right)$.

Note that we here obtain the same expansion of Maxey (1987) reported and discussed in Ferry and Balachandar (2001) and Balachandar and Eaton (2010).

\section{Appendix D: LES filtering approach}

\section{D1 Filtering the equilibrium-Eulerian model}

In order to obtain the LES filtered version of Eq. (15), we apply the Favre filter Eq. (22) to the equilibrium-Eulerian model fundamental Eq. (14) modified as follows:

$\boldsymbol{u}_{j}=\boldsymbol{u}_{\mathrm{g}}+\boldsymbol{w}_{j}-\tau_{j}\left(\partial_{t} \boldsymbol{u}_{\mathrm{m}}+\boldsymbol{u}_{\mathrm{m}} \cdot \nabla \boldsymbol{u}_{\mathrm{m}}+\left(\boldsymbol{w}_{\mathrm{r}}+\boldsymbol{u}_{j}-\boldsymbol{u}_{\mathrm{g}}\right) \cdot \nabla \boldsymbol{u}_{\mathrm{m}}\right)$,

\footnotetext{
${ }^{1}$ For this reason the model is known as equilibrium-Eulerian model.
}

moving the new second-order terms into $O\left(\tau_{j}^{2}\right)$, using $\partial_{t} y_{j}+$ $\boldsymbol{u}_{j} \cdot \nabla y_{j}=0$, defining

$\boldsymbol{w}_{\mathrm{r}}=-\sum_{j} y_{j} \boldsymbol{w}_{j}$

and recalling that at the leading order $\widetilde{\boldsymbol{u}}_{\mathrm{m}} \simeq \widetilde{\boldsymbol{u}}_{\mathrm{g}}-\widetilde{\boldsymbol{w}}_{\mathrm{r}}$. Multiplying the new expression for $\boldsymbol{u}_{j}$ by $\rho_{\mathrm{m}}$ and Favre filtering, at the first order we obtain

$\widetilde{\boldsymbol{u}}_{j}=\widetilde{\boldsymbol{u}}_{\mathrm{g}}+\widetilde{\mathbb{G}}^{-1} \cdot\left[\boldsymbol{w}_{j}-\tau_{j}\left(\widetilde{\boldsymbol{a}}_{\mathrm{m}}+\widetilde{\boldsymbol{w}}_{\mathrm{r}} \cdot \nabla \widetilde{\boldsymbol{u}}_{\mathrm{m}}\right)-\frac{\tau_{j}}{\bar{\rho}_{\mathrm{m}}} \nabla \cdot \mathbb{B}\right]$,

where we have used $\widetilde{\boldsymbol{a}}_{\mathrm{m}}=\partial_{t} \widetilde{\boldsymbol{u}}_{\mathrm{m}}+\widetilde{\boldsymbol{u}}_{\mathrm{m}} \cdot \nabla \widetilde{\boldsymbol{u}}_{\mathrm{m}}, \tilde{\tau}_{j}=\tau_{j}$ and consequently $\widetilde{\boldsymbol{w}}_{j}=\boldsymbol{w}_{j}$ because the Stokes time changes only at the large scale and it can be considered constant at the filter scale. Moreover, we have used the definition subgrid-scale

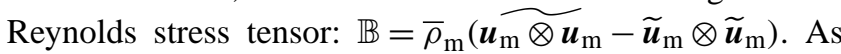
discussed and tested in Shotorban and Balachandar (2007), the subgrid terms can be considered $O\left(\tau_{j}\right)$ and neglected when multiplied by first-order terms.

\section{D2 Filtering the ASHEE model}

To filter the momentum advection term of the ASHEE model (23) we used the Boussinesq eddy viscosity hypothesis Eq. (24c), where the deviatoric part of the subgrid stress tensor can be modeled with an eddy viscosity $\mu_{\mathrm{t}}$ times the rate-of-shear tensor $\widetilde{\mathbb{S}}_{\mathrm{m}}$. On the other hand, the first term on the right-hand side of Eq. (24c) is the isotropic part of the subgrid-scale tensor, proportional to the subgrid-scale kinetic energy $K_{\mathrm{t}}$. While in incompressible turbulence the latter term is absorbed into the pressure, it must be modeled for compressible flows (see Moin et al., 1991; Yoshizawa, 1986). Ducros et al. (1995) showed another way to treat this term by absorbing it into a new macro-pressure and macrotemperature (see also Lesieur et al., 2005; Lodato et al., 2009).

To filter the advection of a generic field $\psi$, we used the eddy diffusivity viscosity model (see Moin et al., 1991): any scalar $\psi$ transported by $\boldsymbol{u}_{\mathrm{m}}$ generates a subgrid-scale vector that can be modeled with the large eddy variables. We have

$\bar{\rho}_{\mathrm{m}}\left(\widetilde{\boldsymbol{u}_{\mathrm{m}} \psi}-\widetilde{\boldsymbol{u}}_{\mathrm{m}} \widetilde{\psi}\right)=-\frac{\mu_{\mathrm{t}}}{P r_{\mathrm{t}}} \nabla \widetilde{\psi}$

where $P r_{\mathrm{t}}$ is the subgrid-scale turbulent Prandtl number.

Moreover, we used additional approximations to filter all the equations. The viscous terms in momentum and energy equations, and the pressure-dilatation and conduction terms in the energy equations are all nonlinear terms and we here treat them as done by Erlebacher et al. (1990) and Moin et al. (1991). The subgrid terms corresponding to the former nonlinear terms could be neglected so that, for example, $\overline{p \nabla \cdot \boldsymbol{u}_{\mathrm{g}}} \simeq \bar{p} \nabla \cdot \widetilde{\boldsymbol{u}}_{\mathrm{g}}$. In particular, this term has been neglected also in presence of shocks (see Garnier et al., 2002). 
We refer to Vreman (1995) for a priori and a posteriori analysis of all the neglected terms of the compressible NavierStokes equations. Moreover, in our model the mixture specific heat $C_{\mathrm{m}}$ and the mixture gas constant $R_{\mathrm{m}}$ vary in the domain because $y_{i}$ and $y_{j}$ vary. Thus, also the following approximations should be done, coherently with the other approximations used: $\widetilde{h}_{\mathrm{m}}=C_{\mathrm{m}} \widetilde{T+p} / \rho_{\mathrm{m}} \simeq \widetilde{C}_{\mathrm{m}} \widetilde{T}+\bar{p} / \bar{\rho}_{\mathrm{m}}$ and $\widetilde{\sum_{\mathcal{I}} y_{i} R_{i}} T \simeq \sum_{\mathcal{I}} \widetilde{y}_{i} R_{i} \widetilde{T}$. 
Acknowledgements. This work includes some results achieved in the PhD work by the first author (MC), carried out at Scuola Normale Superiore, Pisa, with a grant by Istituto Nazionale di Geofisica e Vulcanologia. L. C. Berselli and M. Cerminara are members of GNAMPA, of the Istituto Nazionale di Alta Matematica "F. Severi". We thank A. Neri and M. de' Micheli Vitturi for their suggestions and help about volcanological models, and M. Bernardini and S. Pirozzoli for useful discussion on decaying turbulence and for providing DNS data for model comparison and validation. We thank A. Folch and A. Costa for their thorough reviews which have greatly improved the paper. We acknowledge the CINECA for the availability of high-performance computing resources and technical support on porting OpenFOAM ${ }^{\circledR}$ on HPC architectures by I. Spisso and M. Culpo. In particular, this work took advantage of the CINECA infrastructure through the ISCRA projects: IsB06 VolcFOAM, IsC26 VolcAshP and IsC07 GEOFOAM.

Edited by: S. Marras

\section{References}

Bagheri, G., Bonadonna, C., Manzella, I., Pontelandolfo, P., and Haas, P.: Dedicated vertical wind tunnel for the study of sedimentation of non-spherical particles., Rev. Sci. Instrum., 84, 054501, doi:10.1063/1.4805019, 2013.

Balachandar, S.: A scaling analysis for point-particle approaches to turbulent multiphase flows, Int. J. Multiph. Flow, 35, 801-810, doi:10.1016/j.ijmultiphaseflow.2009.02.013, 2009.

Balachandar, S. and Eaton, J. K.: Turbulent dispersed multiphase flow, Ann. Rev. Fluid Mech., 42, 111-133, doi:10.1146/annurev.fluid.010908.165243, 2010.

Bardina, J., Ferziger, J. H., and Reynolds, W. C.: Improved Subgrid Scale Models for Large Eddy Simulation, 1980, American Institute of Aeronautics and Astronautics, 13th Fluid and Plasma Dynamics Conference, Snowmass, Pap. No. 80, Colo., 14-16 July, 1980.

Bernardini, M. and Pirozzoli, S.: A general strategy for the optimization of Runge-Kutta schemes for wave propagation phenomena, J. Comput. Phys., 228, 4182-4199, 2009.

Berselli, L., Iliescu, T., and Layton, W. J.: Mathematics of Large Eddy Simulation of Turbulent Flows, Springer Science \& Business Media, Springer-Verlag Berlin Heidelberg, Germany, 356 pp., 2005.

Berselli, L. C., Cerminara, M., and Iliescu, T.: Disperse twophase flows, with applications to geophysical problems, Pure Appl. Geophys., 172, 181-196, doi:10.1007/s00024-014-08895, 2015.

Blaisdell, G. A., Mansour, N. N., and Reynolds, W. C.: Numerical Simulation of Compressible Homogeneous Turbulence, Stanford University, Dept. of Mech. Eng., Thermosciences Div. Rep. TF50, 1991.

Boffetta, G., Celani, A., Lillo, F. D., and Musacchio, S.: The Eulerian description of dilute collisionless suspension, EPLEurophys. Lett., 78, 14001, doi:10.1209/0295-5075/78/14001, 2007.

Bonadonna, C., Macedonio, G., and Sparks, R. S. J.: Numerical modelling of tephra fallout associated with dome collapses and Vulcanian explosions: application to hazard assessment on Montserrat, Geol. Soc. London, Mem., 21, 517-537, 2002.

Bürger, R. and Wendland, W. L.: Sedimentation and suspension flows: historical perspective and some recent developments, J. Eng. Math., 41, 101-116, doi:10.1023/A:1011934726111, 2001.

Bursik, M. I.: Effect of wind on the rise height of volcanic plumes, Geophys. Res. Lett., 28, 3621-3624, doi:10.1029/2001GL013393, 2001.

Carcano, S., Bonaventura, L., Esposti Ongaro, T., and Neri, A.: A semi-implicit, second-order-accurate numerical model for multiphase underexpanded volcanic jets, Geosci. Model Dev., 6, 1905-1924, doi:10.5194/gmd-6-1905-2013, 2013.

Cerminara, M.: The Multiphase Buoyant Plume Solution of the Dusty Gas Model, in preparation, ArXiV, available at: http: //arxiv.org/abs/1506.01638 (last access: 12 September 2015), 2015.

Cerminara, M.: Multiphase Flows in Volcanology, $\mathrm{PhD}$ thesis, Scuola Normale Superiore, in preparation, 2016.

Cerminara, M., Berselli, L. C., Esposti Ongaro, T., and Salvetti, M. V.: Direct numerical simulation of a compressible multiphase flow through the Eulerian approach, in: Direct Large-Eddy Simul. IX, edited by: Fröhlich, J., Kuerten, H., Geurts, B. J., and Armenio, V., ERCOFTAC Series, Springer, Switzerland, 2015.

Cerminara, M., Esposti Ongaro, T., and Neri, A.: Large eddy simulation of gas-particle kinematic decoupling and turbulent entrainment in volcanic plumes, J. Volcanol. Geoth. Res., submitted, 2016.

Chacón-Rebollo, T. and Lewandowski, R.: Mathematical and Numerical Foundations of Turbulence Models, Birkhäuser, NewYork, 2013.

Chai, X. and Mahesh, K.: Dynamic-equation model for large-eddy simulation of compressible flows, J. Fluid Mech., 699, 385-413, doi:10.1017/jfm.2012.115, 2012.

Cioni, R., Longo, A., Macedonio, G., Santacroce, R., Sbrana, A., Sulpizio, R., and Andronico, D.: Assessing pyroclastic fall hazard through field data and numerical simulations: example from Vesuvius, J. Geophys. Res.-Sol. Ea., 108, 1-11, doi:10.1029/2001JB000642, 2003.

Clift, R., Grace, J., and Weber, M.: Bubbles, Drops, and Particles, Academic Press, New York, San Francisco, London, 1978.

Costa A., Folch A., and Macedonio G.: Density-driven transport in the umbrella region of volcanic clouds: Implications for tephra dispersion models, Geophys. Res. Lett., 40, 4823-4827, doi:10.1002/grl.50942, 2013.

Costa, A., Suzuki, Y. J., Cerminara, M., Devenish, B. J., Esposti Ongaro, T., Herzog, M., Van Eaton, A. R., Denby, L., Bursik, M. I., de' Michieli Vitturi, M., Engwell, S., Neri, A., Barsotti, S., Folch, A., Macedonio, G., Girault, F., Carazzo, G., Tait, S., Kaminski, E., Mastin, L. G., Woodhouse, M. J., Phillips, J., Hogg, A. J., Degruyter, W., and Bonadonna, C.: Overview of the results of the eruption column model inter-comparison exercise, J. Volcanol. Geoth. Res., submitted, 2015.

Culpo, M.: Current Bottlenecks in the Scalability of OpenFOAM on Massively Parallel Clusters, Tech. rep., PRACE white papers, available at: http://www.prace-ri.eu (last access: 14 October 2015), 2011.

Dagna, P.: OpenFOAM on BG/Q Porting and Performance, Tech. rep., CINECA, available at: http://www.training.prace-ri.eu/ 
uploads/tx_pracetmo/OpenFOAM_on_BGQ_porting_and_ performance.pdf (last access: 14 October 2015), 2013.

Dartevelle, S., Rose, W. I., Stix, J., Kelfoun, K., and Vallance, J. W.: Numerical modeling of geophysical granular flows: 2. Computer simulations of plinian clouds and pyroclastic flows and surges, Geochem. Geophy. Geosy., 5, Q08004, doi:10.1029/2003GC000637, 2004.

Dellino, P., Mele, D., Bonasia, R., Braia, L., La Volpe, L., and Sulpizio, R.: The analysis of the influence of pumice shape on its terminal velocity, Geophys. Res. Lett., 32, L21306, doi:10.1029/ 2005GL023954, 2005.

de' Michieli Vitturi, M., Neri, A., and Barsotti, S.: PLUMEMoM 1.0: A new integral model of volcanic plumes based on the method of moments, Geosci. Model Dev., 8, 2447-2463, doi:10.5194/gmd-8-2447-2015, 2015.

Di Muro, A., Neri, A., and Rosi, M.: Contemporaneous convective and collapsing eruptive dynamics: the transitional regime of explosive eruptions, Geophys. Res. Lett., 31, L10607, doi:10.1029/2004GL019709, 2004.

Dobran, F., Neri, A., and Macedonio, G.: Numerical simulation of collapsing volcanic columns, J. Geophys. Res., 98, 4231-4259, 1993.

Ducros, F., Comte, P., and Lesieur, M.: Large-eddy simulation of a spatially growing boundary layer over an adiabatic flat plate at low Mach number, Int. J. Heat Fluid Flow, 16, 341-348, 1995.

Elghobashi, S.: Particle-laden turbulent flows: direct simulation and closure models, Appl. Sci. Res., 48, 301-314, 1991.

Elghobashi, S.: On predicting particle-laden turbulent flows, Appl. Sci. Res., 52, 309-329, 1994.

Erlebacher, G., Hussaini, M. Y., Speziale, C. G., and Zang, T. A.: Toward the Large-Eddy Simulation of Compressible Turbulent Flows, ICASE Report 90-76, Tech. rep., ICASE/NASA Langley Research Center, 1990.

Esposti Ongaro, T., Neri, A., Menconi, G., de' Michieli Vitturi, M., Marianelli, P., Cavazzoni, C., Erbacci, G., and Baxter, P. J.: Transient 3D numerical simulations of column collapse and pyroclastic density current scenarios at Vesuvius, J. Volcanol. Geotherm. Res., 178, 378-396, 2008.

Fanneløp, T. K. and Webber, D. M.: On buoyant plumes rising from area sources in a calm environment, J. Fluid Mech., 497, 319334, doi:10.1017/S0022112003006669, 2003.

Feireisl, E.: Dynamics of Viscous Compressible Fluids, vol. 26 of Oxford Lecture Series in Mathematics and its Applications, Oxford University Press, Oxford, 212 pp., 2004.

Ferry, J. and Balachandar, S.: A fast Eulerian method for disperse two-phase flow, Int. J. Multiph. Flow, 27, 1199-1226, doi:10.1016/S0301-9322(00)00069-0, 2001.

Ferry, J. and Balachandar, S.: Equilibrium expansion for the Eulerian velocity of small particles, Powder Technol., 125, 131-139, doi:10.1016/S0032-5910(01)00499-5, 2002.

Ferry, J. and Balachandar, S.: Equilibrium Eulerian approach for predicting the thermal field of a dispersion of small particles, Int. J. Heat Mass Trans., 48, 681-689, doi:10.1016/j.ijheatmasstransfer.2004.07.047, 2005.

Ferry, J., Rani, S. L., and Balachandar, S.: A locally implicit improvement of the equilibrium Eulerian method, Int. J. Multiph. Flow, 29, 869-891, doi:10.1016/S0301-9322(03)00064-8, 2003.

Ferziger, J. H. and Perić, M.: Computational Methods for Fluid Dynamics, Vol. 3, Springer, Berlin, 423 pp., 1996.
Folch, A., Costa, A., and Macedonio, G.: FPLUME-1.0: An integrated volcanic plume model accounting for ash aggregation, Geosci. Model Dev. Discuss., 8, 8009-8062, doi:10.5194/gmdd8-8009-2015, 2015.

Fureby, C.: On subgrid scale modeling in large eddy simulations of compressible fluid flow, Phys. Fluids, 8, 1301, doi:10.1063/1.868900, 1996.

Ganser, G.: A rational approach to drag prediction of spherical and nonspherical particles, Powd. Tech., 77, 143152, 1993.

Garnier, E., Mossi, M., Sagaut, P., Comte, P., and Deville, M.: On the use of shock-capturing schemes for large-Eddy simulation, J. Comput. Phys., 153, 273-311, doi:10.1006/jcph.1999.6268, 1999.

Garnier, E., Sagaut, P., and Deville, M.: Large Eddy simulation of shock/homogeneous turbulence interaction, Comput. Fluids, 31, 245-268, doi:10.1016/S0045-7930(01)00022-6, 2002.

Garnier, E., Adams, N., and Sagaut, P.: Large Eddy Simulation for Compressible Flows, doi:10.1007/978-90-481-2819-8, Springer, the Netherlands, 276 pp., 2009.

George, W. K., Alpert, R. L., and Tamanini, F.: Turbulence measurements in an axisymmetric buoyant plume, Int. J. Heat Mass Transf., 20, 1145-1154, doi:10.1016/0017-9310(77)90123-5, 1977.

Germano, M., Piomelli, U., Moin, P., and Cabot, W. H.: A dynamic subgrid-scale eddy viscosity model, Phys. Fluids A-Fluid, 3, 1760-1765, 1991.

Geurts, B. J. and Fröhlich, J.: A framework for predicting accuracy limitations in large-eddy simulation, Phys. Fluids, 14, L41, doi:10.1063/1.1480830, 2002.

Gidaspow, D.: Multiphase Flow and Fluidization: Continuum and Kinetic Theory Descriptions, Academic Press, San Diego, California, 1994.

Glaze, L. S. and Baloga, S. M.: Sensitivity of buoyant plume heights to ambient atmospheric conditions: Implications for volcanic eruption columns, J. Geophys. Res., 101, 1529-1540, 1996.

Graf, H.-F., Herzog, M., Oberhuber, J. M., and Textor, C.: Effect of environmental conditions on volcanic plume rise, J. Geophys. Res., 104, 24309-24320, 1999.

Graham, T.: On the motion of gases, Philos. T. R. Soc., 136, 573$631,1846$.

Greenshields, C. J., Weller, H. G., Gasparini, L., and Reese, J. M.: Implementation of semi-discrete, non-staggered central schemes in a colocated, polyhedral, finite volume framework, for highspeed viscous flows, Int. J. Numer. Methods Fluids, 63, 1-21, doi:10.1002/fld.2069, 2010.

Hashimoto, A., Shimbori, T., and Fukui, K.: Tephra fall simulation for the eruptions at Mt. Shinmoe-dake during 26-27 January 2011 with JMANHM, SOLA, 8, 37-40, 2012.

Honein, A. E. and Moin, P.: Higher entropy conservation and numerical stability of compressible turbulence simulations, J. Comput. Phys., 201, 531-545, doi:10.1016/j.jcp.2004.06.006, 2004.

Ishimine, Y.: Sensitivity of the dynamics of volcanic eruption columns to their shape, Bull. Volcanol., 68, 516-537, doi:10.1007/s00445-005-0027-4, 2006.

Issa, R. I.: Solution of the implicitly discretised fluid flow equations by operator-splitting, J. Comput. Phys., 62, 40-65, doi:10.1016/0021-9991(86)90099-9, 1986. 
Jasak, H.: Error Analysis and Estimation for the Finite Volume Method with Applications to Fluid Flows, PhD thesis, Imperial College London, 394 pp., 1996.

Kaminski, E. and Jaupart, C.: The size distribution of pyroclasts and the fragmentation sequence in explosive volcanic eruptions, J. Geophys. Res., 103, 29759, doi:10.1029/98JB02795, 1998.

Kaminski, E., Tait, S., and Carazzo, G.: Turbulent entrainment in jets with arbitrary buoyancy, J. Fluid Mech., 526, 361-376, doi:10.1017/S0022112004003209, 2005.

Kay, D. A., Gresho, P. M., Griffiths, D. F., and Silvester, D. J.: Adaptive time-stepping for incompressible flow part ii: Navier-stokes equations, SIAM J. Sci. Comput., 32, 111-128, 2010.

Kieffer, S. W.: Factors governing the structure of volcanic jets, Explos. Volcanism: Inception, Evol., Hazards, National Academy, Washington, 143-157, 1984.

Koyaguchi, T., Suzuki, Y. J., and Kozono, T.: Effects of the crater on eruption column dynamics, J. Geophys. Res., 115, B07205, doi:10.1029/2009JB007146, 2010.

Kueppers, U., Perugini, D., and Dingwell, D. B.: "Explosive energy" during volcanic eruptions from fractal analysis of pyroclasts, Earth Planet. Sci. Lett., 248, 800-807, 2006.

Kurganov, A., Noelle, S., and Petrova, G.: Semidiscrete centralupwind schemes for hyperbolic conservation laws and Hamilton-Jacobi equations, SIAM J. Sci. Comput., 23, 707-740, 2001.

Lesieur, M., Métais, O., and Comte, P.: Large-Eddy Simulations of Turbulence, Vol. 1, Cambridge University Press, 2005.

Liao, W., Peng, Y., and Luo, L.-S.: Gas-kinetic schemes for direct numerical simulations of compressible homogeneous turbulence, Phys. Rev. E, 80, 046702, doi:10.1103/PhysRevE.80.046702, 2009.

List, E. J.: Turbulent jets and plumes, Annu. Rev. Fluid Mech., 14, 189-212, doi:10.1146/annurev.fl.14.010182.001201, 1982.

Lodato, G., Vervisch, L., and Domingo, P.: A compressible wall-adapting similarity mixed model for large-eddy simulation of the impinging round jet, Phys. Fluids, 21, 035102, doi:10.1063/1.3068761, 2009.

Marble, F.: Dynamics of dusty gases, Annu. Rev. Fluid Mech., 2, 397-446, doi:10.1146/annurev.fl.02.010170.002145, 1970.

Maxey, M. R.: The gravitational settling of aerosol particles in homogeneous turbulence and random flow fields, J. Fluid Mech., 174, 441, doi:10.1017/S0022112087000193, 1987.

Moin, P., Squires, K., Cabot, W., and Lee, S.: A dynamic subgridscale model for compressible turbulence and scalar transport, Phys. Fluids A-Fluid, 3, 2746, doi:10.1063/1.858164, 1991.

Morrissey, M. M.: Burst conditions of explosive volcanic eruptions recorded on microbarographs, Science, 275, 1290-1293, doi:10.1126/science.275.5304.1290, 1997.

Morton, B. R.: Forced plumes, J. Fluid Mech., 5, 151-163, 1959.

Morton, B. R., Taylor, G., and Turner, J. S.: Turbulent gravitational convection from maintained and instantaneous sources, P. R. Soc. A, 234, 1-23, doi:10.1098/rspa.1956.0011, 1956.

Neri, A. and Dobran, F.: Influence of eruption parameters on the thermofluid dynamics of collapsing volcanic columns, J. Geophys. Res., 99, 11833-11857, doi:10.1029/94JB00471, 1994.

Neri, A., Esposti Ongaro, T., Macedonio, G., and Gidaspow, D.: Multiparticle simulation of collapsing volcanic columns and pyroclastic flow, J. Geophys. Res., 108, 2202, doi:10.1029/2001JB000508, 2003.
Nicoud, F. and Ducros, F.: Subgrid-scale stress modelling based on the square of the velocity gradient tensor, Flow Turbul. Combust., 62, 183-200, doi:10.1023/A:1009995426001, 1999.

Oberhuber, J. M., Herzog, M., Graf, H.-F., and Schwanke, K.: Volcanic plume simulation on large scales, J. Volcanol. Geoth. Res., 87, 29-53, doi:10.1016/S0377-0273(98)00099-7, 1998.

Ogden, D. E., Glatzmaier, G. A., and Wohletz, K. H.: Effects of vent overpressure on buoyant eruption columns: implications for plume stability, Earth Planet Sc. Lett., 268, 283-292, 2008a.

Ogden, D. E., Wohletz, K. H., Glatzmaier, G. A., and Brodsky, E. E.: Numerical simulations of volcanic jets: importance of vent overpressure, J. Geophys. Res., 113, B02204, doi:10.1029/2007JB005133, 2008b.

Orescanin, M. M., Austin, J. M., and Kieffer, S. W.: Unsteady high-pressure flow experiments with applications to explosive volcanic eruptions, J. Geophys. Res., 115, B06206, doi:10.1029/2009JB006985, 2010.

Patankar, S. V.: Numerical Heat Transfer and Fluid Flow, Hemisphere Publishing Corporation, New York, USA, 205 pp., 1980.

Pelanti, M. and LeVeque, R. J.: High-resolution finite volume methods for dusty gas jets and plumes, SIAM J. Sci. Comput., 28, 1335-1360, doi:10.1137/050635018, 2006.

Pfeiffer T., Costa A., Macedonio G.: A model for the numerical simulation of tephra fall deposits, J. Volcanol. Geotherm. Res., 140, 273-294, doi:10.1016/j.jvolgeores.2004.09.001, 2005.

Pirozzoli, S. and Grasso, F.: Direct numerical simulations of isotropic compressible turbulence: influence of compressibility on dynamics and structures, Phys. Fluids, 16, 4386, doi:10.1063/1.1804553, 2004.

Piscaglia, F., Montorfano, A., and Onorati, A.: Towards the LES simulation of IC engines with parallel topologically changing meshes, SAE Int. J. Engines, 6, 926-940, doi:10.4271/2013-011096, 2013.

Plourde, F., Pham, M. V., Kim, S. D., and Balachandar, S.: Direct numerical simulations of a rapidly expanding thermal plume: structure and entrainment interaction, J. Fluid Mech., 604, 99123, doi:10.1017/S0022112008001006, 2008.

Pope, S. B.: Turbulent Flows, Cambridge University Press, 771 pp., 2000.

Prandtl, L.: The Essentials of Fluid Dynamics, doi:10.1007/978-14419-1564-1, Blackie \& Son Limited, New York, 1963.

Rani, S. L. and Balachandar, S.: Evaluation of the equilibrium Eulerian approach for the evolution of particle concentration in isotropic turbulence, Int. J. Multiph. Flow, 29, 1793-1816, doi:10.1016/j.ijmultiphaseflow.2003.09.005, 2003.

Ricou, F. P. and Spalding, D. B.: Measurements of entrainment by axisymmetrical turbulent jets, J. Fluid Mech., 11, 21-32, 1961.

Sagaut, P.: Large Eddy Simulation for Incompressible Flows: an Introduction, Springer Science \& Business Media, Germany, 556 pp., 2006.

Scase, M. M.: Evolution of volcanic eruption columns, J. Geophys. Res., 114, F04003, doi:10.1029/2009JF001300, 2009.

Shabbir, A. and George, W. K.: Experiments on a round turbulent buoyant plume, J. Fluid Mech., 275, 1-32, 1994.

Shotorban, B. and Balachandar, S.: A Eulerian model for large-eddy simulation of concentration of particles with small Stokes numbers, Phys. Fluids, 19, 118107, doi:10.1063/1.2804956, 2007.

Sod, G.: A survey of several of nonlinear finite difference methods hyperbolic conservation laws, J. Comput. Phys., 27, 1-31, 1978. 
Sparks, R. S. J.: The dynamics of bubble formation and growth in magmas: a review and analysis, J. Volcanol. Geoth. Res., 3, 1-37, 1978.

Sparks, R. S. J., Bursik, M. I., Carey, S. N., Gilbert, J. S., Glaze, L. S., Sigurdsson, H., and Woods, A. W.: Volcanic Plumes, John Wiley, Chichester, 590 pp., 1997.

Suzuki, Y. J.: A numerical study of turbulent mixing in eruption clouds using a three-dimensional fluid dynamics model, J. Geophys. Res., 110, B08201, doi:10.1029/2004JB003460, 2005.

Suzuki, Y. J. and Koyaguchi, T.: Numerical determination of the efficiency of entrainment in volcanic eruption columns, Geophys. Res. Lett., 37, L05302, doi:10.1029/2009GL042159, 2010.

Suzuki, Y. J. and Koyaguchi, T.: 3D numerical simulation of volcanic eruption clouds during the 2011 Shinmoe-dake eruptions, Earth Planets Space, 65, 581-589, doi:10.5047/eps.2013.03.009, 2013.

Suzuki, Y. J., Costa, A., Cerminara, M., Esposti Ongaro, T., Herzog, M., Van Eaton, A., Denby, L. C.: Inter-comparison of threedimensional models of volcanic plumes, submitted to J. Volcanol. Geoth. Res., accepted, 2016.

Valentine, G. A. and Wohletz, K. H.: Numerical models of Plinian eruption columns and pyroclastic flows, J. Geophys. Res., 94, 1867-1887, 1989.

Van Eaton, A. R., Mastin, L. G., Herzog, M., Schwaiger, H. F., Schneider, D. J., Wallace, K. L., and Clarke, A. B.: Hail formation triggers rapid ash aggregation in volcanic plumes, Nat. Commun., 6, 7860, doi:10.1038/ncomms8860, 2015.

Van Leer, B.: Towards the ultimate conservative difference scheme, J. Comput. Phys., 135, 229-248, 1997.

Veitch, G. and Woods, A. W.: Particle recycling in volcanic plumes, B. Volcanol., 64, 31-39, 2002.

Vreman, A. W. A.: Direct and Large-Eddy Simulation of the Compressible Turbulent Mixing Layer, $\mathrm{PhD}$ thesis, University of Twente, 1995.

Vuorinen, V., Keskinen, J. P., Duwig, C., and Boersma, B. J.: On the implementation of low-dissipative Runge-Kutta projection methods for time dependent flows using OpenFOAM, Comput. Fluids, 93, 153-163, doi:10.1016/j.compfluid.2014.01.026, 2014.
Wang, L.-P. and Maxey, M. R.: Settling velocity and concentration distribution of heavy particles in homogeneous isotropic turbulence, J. Fluid Mech., 256, 27, doi:10.1017/S0022112093002708, 1993.

Wilson, L.: Explosive volcanic eruptions - III - Plinian eruption columns, Geophys. J. Roy. Astr. S., 45, 543-556, 1976.

Wilson, L.: Relationships between pressure, volatile content and ejecta velocity in three types of volcanic explosion, J. Volcanol. Geoth. Res., 8, 297-313, 1980.

Wilson, L., Sparks, R. S. J., and Walker, G. P. L.: Explosive volcanic eruptions - IV. The control of magma properties and conduit geometry on eruption column behaviour, Geophys. J. Roy. Astr. S., 63, 117-148, 1980.

Woodhouse, M. J., Phillips, J. C., and Hogg, A. J.: Unsteady Turbulent Buoyant Plumes, ArXiV, available at: http://arxiv.org/abs/ 1507.06571 (last access: 23 July 2015), 2015.

Woods, A. W.: The fluid dynamics and thermodynamics of eruption columns, Bull. Volcanol., 50, 169-193, 1988.

Woods, A. W.: Moist convection and the injection of volcanic ash into the atmosphere, J. Geophys. Res., 98, 17617-17627, 1993.

Woods, A. W.: Turbulent plumes in nature, Annu. Rev. Fluid Mech., 42, 391-412, doi:10.1146/annurev-fluid-121108-145430, 2010.

Woods, A. W. and Bower, S. M.: The decompression of volcanic jets in a crater during explosive volcanic eruptions, Earth Planet Sci. Lett., 131, 189-205, 1995.

Woods, A. W. and Bursik, M. I.: Particle fallout, thermal disequilibrium and volcanic plumes, B. Volcanol., 53, 559-570, 1991.

Yoshizawa, A.: Statistical theory for compressible turbulent shear flows, with the application to subgrid modeling, Phys. Fluids, 29, 2152, doi:10.1063/1.865552, 1986.

Yoshizawa, A.: Bridging between eddy-viscosity-type and secondorder turbulence models through a two-scale turbulence theory, Phys. Rev. E, 48, 273-281, doi:10.1103/PhysRevE.48.273, 1993.

Yüceil, K. B. and Ötügen, M. V.: Scaling parameters for underexpanded supersonic jets, Phys. Fluids, 14, 4206, doi:10.1063/1.1513796, 2002.

Zhou, X., Luo, K. H., and Williams, J. J.: Large-eddy simulation of a turbulent forced plume, Eur. J. Mech. B-Fluid., 20, 233-254, doi:10.1016/S0997-7546(00)01117-1, 2001. 